TITLE:

\title{
Pullbacks of Hermitian Maass lifts
}

$\operatorname{AUTHOR}(S)$ :

Atobe, Hiraku

\section{CITATION:}

Atobe, Hiraku. Pullbacks of Hermitian Maass lifts. Journal of Number Theory 2015, 153: 158-229

ISSUE DATE:

2015-03-04

URL:

http://hdl.handle.net/2433/198810

\section{RIGHT:}

(c) 2015 Elsevier Inc. Licensed under the Creative Commons Attribution-NonCommercial-NoDerivatives 4.0 International http://creativecommons.org/licenses/by-nc-nd/4.0/. NOTICE: this is the author's version of a work that was accepted for publication in Journal of Number Theory. Changes resulting from the publishing process, such as peer review, editing, corrections, structural formatting, and other quality control mechanisms may not be reflected in this

document. Changes may have been made to this work since it was submitted for publication. A definitive version was subsequently published in Journal of Number Theory, Volume 153, Pages 158-229. DOI:10.1016/j.jnt.2015.01.004.; 許諾 条件により本文ファイルは2017-03-07に公開,; この論文は出版社版でありません。引用の際には出版社版をご確認ご 利用ください。; This is not the published version. Please cite only the published version. 


\title{
PULLBACKS OF HERMITIAN MAASS LIFTS
}

\author{
HIRAKU ATOBE
}

\begin{abstract}
We consider pullbacks of hermitian Maass lifts of degree 2 to the submanifold of diagonal matrices. By using these pullbacks, we give an explicit formula for central values of $L$-functions for $\mathrm{GL}(2) \times \mathrm{GL}(2)$.
\end{abstract}

\section{INTRODUCTION}

Pullbacks of Siegel Eisenstein series have been studied by Böcherer [1], Garrett [8] and Heim [13]. Pullbacks of hermitian Eisenstein series have been studied by Furusawa [5], Harris [10] and Saha [23]. These pullbacks have been used to study the algebraicity of critical values of certain automorphic $L$-functions. Moreover, one might consider pullbacks of cusp forms. The (Gan-)Gross-Prasad conjecture [9], [6] would relate critical values of certain $L$-functions and the pullbacks of an automorphic representation of $\mathrm{SO}(n+1)$ to $\mathrm{SO}(n)$ or one of $\mathrm{U}(n+1)$ to $\mathrm{U}(n)$. For example, in [26], [15] and [7], the pullbacks of an automorphic representation of $\mathrm{SO}(n+1)$ to $\mathrm{SO}(n)$ for small $n$ were studied. In [27] and [28], Zhang studied the Gan-GrossPrasad conjecture for $\mathrm{U}(n+1)$ to $\mathrm{U}(n)$ for general $n$ assuming some additional conditions. On the other hand, Ichino [14] gave an explicit formula for pullbacks of Saito-Kurokawa lifts, which are Siegel cusp forms of degree 2, in terms of central critical values of $L$-functions for $\mathrm{SL}_{2} \times \mathrm{GL}_{2}$. Ichino and Ikeda [16] gave an explicit formula for the restriction of hermitian Maass lifts of degree 2 to the Siegel upper half space of degree 2 in terms of central critical values of triple product $L$-functions. These results may be also regarded as special cases of the Gross-Prasad conjecture. In this paper, we relate pullbacks of hermitian Maass lifts of degree 2 to central values of $L$-functions for $\mathrm{GL}_{2} \times \mathrm{GL}_{2}$.

Let us describe our results. Let $K=\mathbb{Q}(\sqrt{-D})$ be an imaginary quadratic field with discriminant $-D<0$. We denote the ideal class group of $K$ by $C l_{K}$ and the class number of $K$ by $h_{K}$. The primitive Dirichlet character corresponding to $K / \mathbb{Q}$ is denoted by $\chi$. Let $\kappa$ be a positive integer and $f \in S_{2 \kappa+1}\left(\Gamma_{0}(D), \chi\right)$ be a normalized Hecke eigenform. For an integral ideal $\mathfrak{c}$ of $K$ which is prime to $D$, we denote by $F_{\mathfrak{c}}$ the hermitian Maass lift of $f$ which satisfies the Maass relation for $\mathfrak{c}$. The lift $F_{\mathfrak{c}}$ is an automorphic form on the hermitian upper half space $\mathcal{H}_{2}$ of degree 2 with respect to a certain arithmetic subgroup $\Gamma_{K}^{(2)}[\mathfrak{c}] \subset \mathrm{U}(2,2)(\mathbb{Q})$. See Sect. 2 for details. Let $C=N(\mathfrak{c})$ be the ideal norm of $\mathfrak{c}$ and $d(C)=\operatorname{diag}(1, C) \in \mathrm{GL}_{2}(\mathbb{Q})$. The pullback $\left.F_{\mathfrak{c}}\right|_{\mathfrak{H} \times \mathfrak{H}}$ is in $S_{2 \kappa+2}\left(\mathrm{SL}_{2}(\mathbb{Z})\right) \otimes S_{2 \kappa+2}\left(d(C)^{-1} \mathrm{SL}_{2}(\mathbb{Z}) d(C)\right)$. For each normalized Hecke eigenform $g \in S_{2 \kappa+2}\left(\mathrm{SL}_{2}(\mathbb{Z})\right)$, we put $g_{C}(z)=g(z / C) \in S_{2 \kappa+2}\left(d(C)^{-1} \mathrm{SL}_{2}(\mathbb{Z}) d(C)\right)$ and consider the period integral $\left\langle\left. F_{\mathfrak{c}}\right|_{\mathfrak{H} \times \mathfrak{H}}, g \times g_{C}\right\rangle$ given by

$$
\begin{aligned}
& \left\langle\left. F_{\mathfrak{c}}\right|_{\left.\mathfrak{H} \times \mathfrak{H}, g \times g_{C}\right\rangle}=\int_{d(C)^{-1} \mathrm{SL}_{2}(\mathbb{Z}) d(C) \backslash \mathfrak{H}} \int_{\mathrm{SL}_{2}(\mathbb{Z}) \backslash \mathfrak{H}} F_{\mathfrak{c}}\left(\left(\begin{array}{cc}
z_{1} & 0 \\
0 & z_{2}
\end{array}\right)\right) \overline{g\left(z_{1}\right) g_{C}\left(z_{2}\right)} y_{1}^{2 \kappa} y_{2}^{2 \kappa} d z_{1} d z_{2} .\right.
\end{aligned}
$$

Let $L(s, f \times g)$ and $L(s, f \times g \times \chi)$ be the Rankin-Selberg $L$-function and its twist given by $f$ and $g$ of degree 4 . We put $L_{\infty}(s)=\Gamma_{\mathbb{C}}(s+2 \kappa+1 / 2) \Gamma_{\mathbb{C}}(s+1 / 2)$ with $\Gamma_{\mathbb{C}}(s)=2(2 \pi)^{-s} \Gamma(s)$. They satisfy the functional equation

$$
L_{\infty}(s) L(s, f \times g)=-D^{1-2 s+2 \kappa} a_{f}(D)^{-2} L_{\infty}(1-s) L(1-s, f \times g \times \chi),
$$

Key words and phrases. automorphic form; special value of $L$-functions; period. 
where $a_{f}(D)$ is the $D$-th Fourier coefficient of $f$. Let $L(s, \chi)$ be the Dirichlet $L$-function associated with $\chi$.

Our main result is as follows.

Theorem 3.2. The identity

$$
L\left(\frac{1}{2}, f \times g\right)=\frac{L(1, \chi)(4 \pi)^{2 \kappa+1}}{a_{f}(D)(2 \kappa) !} \cdot \frac{1}{h_{K}} \sum_{[\mathfrak{c}] \in C l_{K}} \frac{\left\langle\left. F_{\mathfrak{c}}\right|_{\mathfrak{H} \times \mathfrak{H}}, g \times g_{C}\right\rangle}{\left\langle g_{C}, g_{C}\right\rangle}
$$

holds.

Note that the period integrals do not appear in the (Gan-)Gross-Prasad conjecture. We remark that the period integrals appearing in the right hand side are not square. This is a difference to the results of [14] or [16], which relate the central critical values of certain $L$ functions to the square of the absolute values of period integrals.

The sketch of proof is as follows. The lifts $\left\{F_{\mathfrak{c}}\right\}$ give an automorphic form Lift $^{(2)}(f)$ on $\mathrm{U}(2,2)\left(\mathbb{A}_{\mathbb{Q}}\right)$. We consider the restriction of $\operatorname{Lift}^{(2)}(f)$ to $(\mathrm{U}(1,1) \times \mathrm{U}(1,1))\left(\mathbb{A}_{\mathbb{Q}}\right)$. The group $\mathrm{U}(2,2)$ is closely related to $\mathrm{O}(4,2)$ (see Sect. 6). So we may regard a certain theta lift on $\mathrm{O}(4,2)\left(\mathbb{A}_{\mathbb{Q}}\right)$ as a function on $\mathrm{U}(2,2)\left(\mathbb{A}_{\mathbb{Q}}\right)$. First, we prove that the theta lift is equal to Lift $^{(2)}(f)$ up to scalar multiplication (Proposition 6.2). The group $\mathrm{U}(1,1) \times \mathrm{U}(1,1)$ is closely related to $\mathrm{O}(2,2) \times \mathrm{O}(2)$ and the function $g \times g_{C}$ gives an automorphic form on $\mathrm{O}(2,2)\left(\mathbb{A}_{\mathbb{Q}}\right)$ (see Sect. 7). Therefore, by the following seesaw identity, we find that a sum of period integrals of $\left\{F_{\mathfrak{c}}\right\}$ is equal to a sum of central values of $L(s, f \times g)$ and its twists (Corollary 9.7).

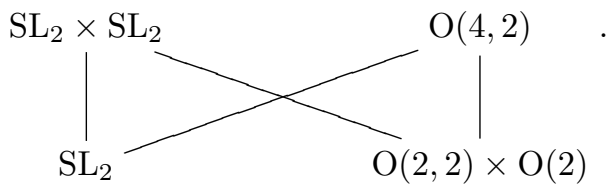

Finally, we show that these equations and the genus theory imply the main theorem.

This paper is organized as follows. In Sect. 2, we review the theory of hermitian Maass lifts. In Sect. 3, we state our main result. In Sects. 4 and 5, we recall the basic facts about automorphic forms on $\mathrm{GL}_{2}$ and theta lifts, respectively. In Sect. 6, we study the hermitian Maass lifting. In Sects. 7 and 8, we recall the theta correspondence for $\left(\mathrm{GL}_{2}, \mathrm{GO}(2,2)\right)$ and $\left(\mathrm{SL}_{2}, \mathrm{O}(2)\right)$, respectively. In Sect. 9, we prove identities for the above seesaw and we show that these identities and the genus theory imply the main result.

Acknowledgments. The author would like to thank my advisor, Prof. Atsushi Ichino. Without his helpful support, this work would not have been completed. The author is also thankful to Prof. Tamotsu Ikeda for useful discussions.

Notation. Let $K=\mathbb{Q}(\sqrt{-D})$ be an imaginary quadratic field with discriminant $-D<0$. We denote by $\mathfrak{o}$ the ring of integers of $K$. Let $x \mapsto \bar{x}$ be the non-trivial Galois automorphism of $K$ over $\mathbb{Q}$. The primitive Dirichlet character corresponding to $K / \mathbb{Q}$ is denoted by $\chi$. We regard $K^{1}=\left\{\alpha \in K^{\times} \mid N_{K / \mathbb{Q}}(\alpha)=1\right\}$ as an algebraic group over $\mathbb{Q}$. We denote by $J_{K}^{D}$ (resp. $J_{\mathfrak{o}}^{D}$ ) the set of fractional ideals (resp. integral ideals) of $K$ which are prime to $D$. Here, we say that a fractional ideal $\mathfrak{c}$ is prime to $D$ if $\operatorname{ord}_{\mathfrak{p}}(\mathfrak{c})=0$ for each prime ideal $\mathfrak{p} \mid D$. Let $C l_{K}$ be the ideal class group of $K$ and $h_{K}=\# C l_{K}$ the ideal class number of $K$.

We define the algebraic group $\mathrm{Her}_{2}$ of hermitian matrices of size 2 with entries in $K$ by

$$
\operatorname{Her}_{2}(R)=\left\{\left(\begin{array}{cc}
a & b+\sqrt{-D} c \\
b-\sqrt{-D} c & d
\end{array}\right) \mid a, b, c, d \in R\right\}
$$

for any $\mathbb{Q}$-algebra $R$.

For a number field $F$, we denote the adele ring of $F$ by $\mathbb{A}_{F}$. The finite part of the adele ring (resp. the idele group) of $F$ is denoted by $\mathbb{A}_{F, \text { fin }}\left(\right.$ resp. $\mathbb{A}_{F, \text { fin }}^{\times}$). Let $\psi_{0}=\otimes_{v} \psi_{v}$ be the non-trivial additive character of $\mathbb{A}_{\mathbb{Q}} / \mathbb{Q}$ defined as follows:

- If $v=p$, then $\psi_{p}(x)=e^{-2 \pi \sqrt{-1} x}$ for $x \in \mathbb{Z}\left[p^{-1}\right]$. 
- If $v=\infty$, then $\psi_{\infty}(x)=e^{2 \pi \sqrt{-1} x}$ for $x \in \mathbb{R}$.

We call $\psi_{0}\left(\right.$ resp. $\left.\psi_{v}\right)$ the standard additive character of $\mathbb{A}_{\mathbb{Q}}\left(\right.$ resp. $\left.\mathbb{Q}_{v}\right)$. We put $\hat{\mathbb{Z}}=\prod_{p} \mathbb{Z}_{p}$.

Let $\underline{\chi}=\otimes_{v} \underline{\chi}_{v}$ be the character of the idele class group $\mathbb{A}_{\mathbb{Q}}^{\times} / \mathbb{Q}^{\times}$determined by $\chi$. Then $\underline{\chi}_{v}$ is the character of $\mathbb{Q}_{v}^{\times}$corresponding to $\mathbb{Q}_{v}(\sqrt{-D}) / \mathbb{Q}_{v}$ and is given by the Hilbert symbol $\underline{\chi}_{v}(x)=(-D, x)_{\mathbb{Q}_{v}}$.

Let

$$
B=\left\{\left(\begin{array}{ll}
* & * \\
0 & *
\end{array}\right) \in \mathrm{SL}_{2}\right\} \quad \text { and } \quad N=\left\{n(x)=\left(\begin{array}{ll}
1 & x \\
0 & 1
\end{array}\right) \mid x \in \mathbb{G}_{a}\right\}
$$

be the standard Borel subgroup of $\mathrm{SL}_{2}$ and the unipotent radical of $B$, respectively. We write

$$
a(x)=\left(\begin{array}{ll}
x & 0 \\
0 & 1
\end{array}\right), \quad d(x)=\left(\begin{array}{ll}
1 & 0 \\
0 & x
\end{array}\right), \quad t(x)=\left(\begin{array}{cc}
x & 0 \\
0 & x^{-1}
\end{array}\right)
$$

and

$$
\operatorname{diag}(x, y)=\left(\begin{array}{ll}
x & 0 \\
0 & y
\end{array}\right), \quad k_{\theta}=\left(\begin{array}{cc}
\cos \theta & \sin \theta \\
-\sin \theta & \cos \theta
\end{array}\right) .
$$

We put $\mathrm{GL}_{2}(\mathbb{R})^{+}=\left\{g \in \mathrm{GL}_{2}(\mathbb{R}) \mid \operatorname{det}(g)>0\right\}$. For $N \in \mathbb{Z}_{p}$, we define

$$
\mathbf{K}_{0}\left(N ; \mathbb{Z}_{p}\right)=\left\{\left(\begin{array}{ll}
a & b \\
c & d
\end{array}\right) \in \mathrm{GL}_{2}\left(\mathbb{Z}_{p}\right) \mid c \in N \mathbb{Z}_{p}\right\}
$$

and $K_{0}\left(N ; \mathbb{Z}_{p}\right)=\mathbf{K}_{0}\left(N ; \mathbb{Z}_{p}\right) \cap \mathrm{SL}_{2}\left(\mathbb{Z}_{p}\right)$.

Let $\mathfrak{H}=\{z \in \mathbb{C} \mid \operatorname{Im}(z)>0\}$ be the complex upper half plane. For $z=x+\sqrt{-1} y \in \mathfrak{H}$, we put $d z=d x d y$ and $q=e^{2 \pi \sqrt{-1} z}$. Here $d x, d y$ are the Lebesgue measures. Note that $\operatorname{vol}\left(\mathrm{SL}_{2}(\mathbb{Z}) \backslash \mathfrak{H}, y^{-2} d z\right)=\pi / 3$.

For an algebraic group $G$ over $\mathbb{Q}$, we put $[G]=G(\mathbb{Q}) \backslash G\left(\mathbb{A}_{\mathbb{Q}}\right)$.

We put $\Gamma_{\mathbb{R}}(s)=\pi^{-s / 2} \Gamma(s / 2), \Gamma_{\mathbb{C}}(s)=2(2 \pi)^{-s} \Gamma(s)$ and $\xi_{\mathbb{Q}}(s)=\Gamma_{\mathbb{R}}(s) \zeta(s)$.

Measures. Let $d x_{\infty}$ be the Lebesgue measure on $\mathbb{R}$. For each prime $p$, let $d x_{p}$ be the Haar measure on $\mathbb{Q}_{p}$ with $\operatorname{vol}\left(\mathbb{Z}_{p}, d x_{p}\right)=1$. We take the Haar measure $d^{\times} x_{v}=\left|x_{v}\right|_{v}^{-1} d x_{v}$ on $\mathbb{Q}_{v}^{\times}$.

We normalize the Haar measures on $\mathrm{SL}_{2}\left(\mathbb{Z}_{p}\right)$ and $\mathrm{SO}(2)$ so that the total volumes are equal to 1 . For a place $v$ of $\mathbb{Q}$, we define a Haar measure $d g_{v}$ on $\mathrm{SL}_{2}\left(\mathbb{Q}_{v}\right)$ by

$$
d g_{v}=\left|a_{v}\right|_{v}^{-2} d x_{v} d^{\times} a_{v} d k_{v}
$$

for $g_{v}=n\left(x_{v}\right) t\left(a_{v}\right) k_{v}$ with $x_{v} \in \mathbb{Q}_{v}, a_{v} \in \mathbb{Q}_{v}^{\times}$, and

$$
k_{v} \in \begin{cases}\mathrm{SL}_{2}\left(\mathbb{Z}_{p}\right) & \text { if } v=p, \\ \mathrm{SO}(2) & \text { if } v=\infty .\end{cases}
$$

We take the product measure $d g=\prod_{v} d g_{v}$ on $\mathrm{SL}_{2}\left(\mathbb{A}_{\mathbb{Q}}\right)$. Note that the measure $\xi_{\mathbb{Q}}(2)^{-1} d g$ is the Tamagawa measure on $\mathrm{SL}_{2}\left(\mathbb{A}_{\mathbb{Q}}\right)$. For another connected linear algebraic group $G$ over $\mathbb{Q}$, we take the Tamagawa measure on $G\left(\mathbb{A}_{\mathbb{Q}}\right)$. In particular, for a quadratic space $V$ over $\mathbb{Q}$ which is neither a hyperbolic plane nor $\operatorname{dim}(V)=1$, we have $\operatorname{vol}([\operatorname{SO}(V)])=2$. We normalize the Haar measure on $\mathrm{O}(V)\left(\mathbb{A}_{\mathbb{Q}}\right)$ so that $\operatorname{vol}([\mathrm{O}(V)])=1$.

\section{HeRmitian MaAss LifTS}

In this section, we review the theory of hermitian modular forms and hermitian Maass lifts. See [17].

2.1. Hermitian modular forms. The similitude unitary group $\mathrm{GU}(2,2)$ is an algebraic group over $\mathbb{Q}$ defined by

$$
\mathrm{GU}(2,2)(R)=\left\{\left.g \in \mathrm{GL}_{4}(K \otimes R)\right|^{t} \bar{g} J g=\lambda(g) J, \lambda(g) \in R^{\times}\right\}
$$

with

$$
J=\left(\begin{array}{cc}
0 & -\mathbf{1}_{2} \\
\mathbf{1}_{2} & 0
\end{array}\right) \in \mathrm{GL}_{4}
$$


for any $\mathbb{Q}$-algebra $R$. The homomorphism $\lambda: \mathrm{GU}(2,2) \rightarrow \mathrm{GL}_{1}$ is called the similitude norm. Let $\mathrm{U}(2,2)=\operatorname{ker}(\lambda)$ be the unitary group and $\mathrm{SU}(2,2)=\mathrm{U}(2,2) \cap \operatorname{Res}_{K / \mathbb{Q}}\left(\mathrm{SL}_{4}\right)$ the special unitary group.

We define the hermitian upper half space $\mathcal{H}_{2}$ of degree 2 by

$$
\mathcal{H}_{2}=\left\{Z \in \mathrm{M}_{2}(\mathbb{C}) \mid \frac{1}{2 \sqrt{-1}}\left(Z-{ }^{t} \bar{Z}\right)>0\right\} .
$$

For a fractional ideal $\mathfrak{c}$ of $K$, we define a subgroup $\Gamma_{K}^{(2)}[\mathfrak{c}]$ of $\mathrm{U}(2,2)(\mathbb{Q})$ by

$$
\Gamma_{K}^{(2)}[\mathfrak{c}]=\left\{g \in \mathrm{U}(2,2)(\mathbb{Q}) \mid g\left(\begin{array}{c}
\mathfrak{o} \\
\mathfrak{c} \\
\mathfrak{o} \\
\overline{\mathfrak{c}}^{-1}
\end{array}\right)=\left(\begin{array}{c}
\mathfrak{o} \\
\mathfrak{c} \\
\mathfrak{o} \\
\overline{\mathfrak{c}}^{-1}
\end{array}\right)\right\},
$$

where $\overline{\mathfrak{c}}$ is the conjugate ideal of $\mathfrak{c}$. Let $C=N(\mathfrak{c}) \in \mathbb{Q}_{>0}$ be the ideal norm of $\mathfrak{c}$. We put

$$
\Lambda_{2}^{\mathfrak{c}}(\mathfrak{o})=\left\{\left(\begin{array}{cc}
n & \alpha \\
\bar{\alpha} & m / C
\end{array}\right) \in \operatorname{Her}_{2}(\mathbb{Q}) \mid n, m \in \mathbb{Z}, \alpha \in \sqrt{-D}^{-1} \mathfrak{c}^{-1}\right\} .
$$

The set of positive definite elements of $\Lambda_{2}^{\mathfrak{c}}(\mathfrak{o})$ is denoted by $\Lambda_{2}^{\mathfrak{c}}(\mathfrak{o})^{+}$.

Let $\mathrm{GU}(2,2)(\mathbb{R})^{+}=\{g \in \mathrm{GU}(2,2)(\mathbb{R}) \mid \lambda(g)>0\}$. Note that $\mathrm{GU}(2,2)(\mathbb{R})^{+}$is generated by its center and $\mathrm{U}(2,2)(\mathbb{R})$. We put

$$
g\langle Z\rangle=(A Z+B)(C Z+D)^{-1} \quad \text { and } \quad j(g, Z)=\operatorname{det}(C Z+D)
$$

for $Z \in \mathcal{H}_{2}$ and

$$
g=\left(\begin{array}{ll}
A & B \\
C & D
\end{array}\right) \in \mathrm{GU}(2,2)(\mathbb{R})^{+} .
$$

We find that $g\langle\cdot\rangle$ gives an action of $\mathrm{GU}(2,2)(\mathbb{R})^{+}$on $\mathcal{H}_{2}$ and the center of $\mathrm{GU}(2,2)(\mathbb{R})^{+}$acts trivially. For a holomorphic function $F$ on $\mathcal{H}_{2}$, an even integer $l$ and $g \in \mathrm{GU}(2,2)(\mathbb{R})^{+}$, we define

$$
\left(F \|_{l} g\right)(Z)=\operatorname{det}(g)^{l / 2} F(g\langle Z\rangle) j(g, Z)^{-l} .
$$

We put

$$
M_{l}\left(\Gamma_{K}^{(2)}[\mathfrak{c}], \operatorname{det}^{-l / 2}\right)=\left\{F \mid F \|_{l} \gamma=F \text { for any } \gamma \in \Gamma_{K}^{(2)}[\mathfrak{c}]\right\} .
$$

Then $F \in M_{l}\left(\Gamma_{K}^{(2)}[\mathfrak{c}], \operatorname{det}^{-l / 2}\right)$ has a Fourier expansion of the form

$$
F(Z)=\sum_{H \in \Lambda_{2}^{c}(\mathfrak{o}), H \geq 0} A(H) \exp (2 \pi \sqrt{-1} \operatorname{Tr}(H Z)) .
$$

The space of cusp forms $S_{l}\left(\Gamma_{K}^{(2)}[\mathfrak{c}], \operatorname{det}^{-l / 2}\right)$ is defined by

$$
S_{l}\left(\Gamma_{K}^{(2)}[\mathfrak{c}], \operatorname{det}^{-l / 2}\right)=\left\{F \in M_{l}\left(\Gamma_{K}^{(2)}[\mathfrak{c}], \operatorname{det}^{-l / 2}\right) \mid A(H)=0 \text { unless } H \in \Lambda_{2}^{\mathfrak{c}}(\mathfrak{o})^{+}\right\} .
$$

2.2. Hermitian Maass lifts. For $x \in \mathbb{Q}^{\times}$and each prime $p$, we put

$$
x^{\prime}=\prod_{p \nmid D} p^{\operatorname{ord}_{p}(x)} \quad \text { and } \quad x_{p}=p^{\operatorname{ord}_{p}(x)} .
$$

Let $Q_{D}$ be the set of all primes which divide $D$. We define a primitive Dirichlet character $\chi_{p}$ by

$$
\chi_{p}(n)= \begin{cases}\chi(m) & \text { if }(n, p)=1, \\ 0 & \text { if } p \mid n,\end{cases}
$$

where $m$ is an integer such that

$$
m \equiv \begin{cases}n & \bmod D_{p} \\ 1 & \bmod D_{p}^{-1} D .\end{cases}
$$


One should not confuse $\chi_{p}$ with $\underline{\chi}_{p}$. For $Q \subset Q_{D}$, we set

$$
\chi_{Q}=\prod_{p \in Q} \chi_{p} \text { and } \chi_{Q}^{\prime}=\prod_{p \in Q_{D} \backslash Q} \chi_{p} .
$$

Note that $\chi_{\emptyset}=1$ and $\chi_{Q_{D}}=\chi$.

Let $\kappa$ be a positive integer. We fix a normalized Hecke eigenform $f=\sum_{n>0} a_{f}(n) q^{n} \in$ $S_{2 \kappa+1}\left(\Gamma_{0}(D), \chi\right)$. By Theorem 4.6.16 of [20], for each subset $Q \subset Q_{D}$, there exists a normalized Hecke eigenform

$$
f_{Q}=\sum_{n>0} a_{f_{Q}}(n) q^{n} \in S_{2 \kappa+1}\left(\Gamma_{0}(D), \chi\right)
$$

such that for each prime $p$, the Fourier coefficient $a_{f_{Q}}(p)$ satisfies

$$
a_{f_{Q}}(p)= \begin{cases}\chi_{Q}(p) a_{f}(p) & \text { if } p \notin Q, \\ \chi_{Q}^{\prime}(p) \overline{a_{f}(p)} & \text { if } p \in Q .\end{cases}
$$

We fix $\mathfrak{c} \in J_{\mathfrak{o}}^{D}$ and put $C=N(\mathfrak{c}) \in \mathbb{Z}_{>0}$. Note that $\chi(C)=1$. Following [17] Definition 15.2, we define

$$
f^{\mathfrak{*} *}=\sum_{Q \subset Q_{D}} \chi_{Q}(-C) f_{Q}
$$

Lemma 2.1. The Fourier coefficients of $f^{\mathfrak{c} *}$ are purely imaginary.

Proof. In the case when $\mathfrak{c}=\mathfrak{o}$, the assertion is Lemma 1.1 of [16]. Let $Q \subset Q_{D}$ and put $Q^{\prime}=Q_{D} \backslash Q$. Then we find that

$$
a_{f_{Q^{\prime}}}(n)=\overline{a_{f_{Q}}(n)}
$$

for all $n \in \mathbb{Z}_{>0}$. Since $(D,-C)=1$, we have $\chi_{Q^{\prime}}(-C)=\chi(-C) \chi_{Q}(-C)^{-1}=\chi(-1) \chi_{Q}(-C)=$ $-\chi_{Q}(-C)$. Therefore, the Fourier coefficients of $\chi_{Q}(-C) f_{Q}+\chi_{Q^{\prime}}(-C) f_{Q^{\prime}}$ are purely imaginary.

By [17] Corollary 15.5, the $n$-th Fourier coefficient of $f^{\mathfrak{c} *}$ is given by

$$
a_{f^{\mathfrak{} *}}(n)=\mathbf{a}_{D}^{\mathfrak{c}}(n) \alpha_{F_{\mathfrak{c}}}(n),
$$

where

$$
\mathbf{a}_{D}^{\mathfrak{c}}(n)=\prod_{p \mid D}\left(1+\chi_{p}(-C n)\right), \quad \alpha_{F_{\mathfrak{c}}}(n)=a_{f}\left(n^{\prime}\right) \prod_{p \mid(D, n)}\left(a_{f}\left(n_{p}\right)+\underline{\chi}_{p}(-C n) \overline{a_{f}\left(n_{p}\right)}\right) .
$$

By [17] $\$ 16$, we can define $F_{\mathfrak{c}} \in S_{2 \kappa+2}\left(\Gamma_{K}^{(2)}[\mathfrak{c}], \operatorname{det}^{-\kappa-1}\right)$ by

$$
F_{\mathfrak{c}}(Z)=\sum_{H \in \Lambda_{2}^{\mathfrak{c}}(\mathfrak{o})^{+}}\left(\sum_{d \mid \varepsilon(H)} d^{2 \kappa+1} \alpha_{F_{\mathfrak{c}}}\left(\frac{C D \operatorname{det}(H)}{d^{2}}\right)\right) \exp (2 \pi \sqrt{-1} \operatorname{Tr}(H Z)) .
$$

Here

$$
\varepsilon(H)=\varepsilon_{\mathfrak{c}}(H)=\max \left\{m \in \mathbb{Z}_{>0} \mid m^{-1} H \in \Lambda_{2}^{\mathfrak{c}}(\mathfrak{o})\right\} .
$$

We call $F_{\mathfrak{c}}$ the hermitian Maass lift of $f$ which satisfies the Maass relation for $\mathfrak{c}$.

Lemma 2.2. Let $\mathfrak{c} \in J_{\mathfrak{o}}^{D}$ and $\alpha \in K^{\times}$such that $\alpha \mathfrak{c} \in J_{\mathfrak{o}}^{D}$. Then

$$
F_{\mathfrak{c}}(Z)=F_{\alpha \mathfrak{c}}(d(\alpha) Z d(\bar{\alpha})) .
$$

Proof. Since ${ }^{t} \overline{(d(\alpha) Z d(\bar{\alpha}))}=d(\alpha)^{t} \bar{Z} d(\bar{\alpha})$, we find that $d(\alpha) Z d(\bar{\alpha}) \in \mathcal{H}_{2}$ for all $Z \in \mathcal{H}_{2}$. Note that $\operatorname{Tr}(H d(\alpha) Z d(\bar{\alpha}))=\operatorname{Tr}(d(\bar{\alpha}) H d(\alpha) Z)$. The map

$$
H \mapsto d(\bar{\alpha}) H d(\alpha)
$$

gives a bijection $\Lambda_{2}^{\alpha \mathfrak{c}}(\mathfrak{o})^{+} \rightarrow \Lambda_{2}^{\mathfrak{c}}(\mathfrak{o})^{+}$which satisfies

$$
\varepsilon_{\mathfrak{c}}(d(\bar{\alpha}) H d(\alpha))=\varepsilon_{\alpha \mathfrak{c}}(H) \text { and } \quad N(\alpha \mathfrak{c}) D \operatorname{det}(H)=N(\mathfrak{c}) D \operatorname{det}(d(\bar{\alpha}) H d(\alpha)) .
$$

This completes the proof. 
By Lemma 2.2, we can define $F_{\mathfrak{c}} \in S_{2 \kappa+2}\left(\Gamma_{K}^{(2)}[\mathfrak{c}]\right.$, det $\left.{ }^{-\kappa-1}\right)$ for a fractional ideal $\mathfrak{c} \in J_{K}^{D}$ by the same formula. Moreover the function

$$
\mathfrak{H} \times \mathfrak{H} \ni\left(z_{1}, z_{2}\right) \mapsto F_{\mathfrak{c}}\left(\operatorname{diag}\left(z_{1}, C z_{2}\right)\right)
$$

depends only on the ideal class of $\mathfrak{c}$ and defines an element in $S_{2 \kappa+2}\left(\mathrm{SL}_{2}(\mathbb{Z})\right) \otimes S_{2 \kappa+2}\left(\mathrm{SL}_{2}(\mathbb{Z})\right)$.

Lemma 2.3. Let $T(p)$ be the Hecke operator on $S_{2 \kappa+2}\left(\mathrm{SL}_{2}(\mathbb{Z})\right)$. Then

$$
(T(p) \otimes \mathrm{id})\left(F_{\mathfrak{c}}\left(\operatorname{diag}\left(z_{1}, C z_{2}\right)\right)\right)=(\mathrm{id} \otimes T(p))\left(F_{\mathfrak{c}}\left(\operatorname{diag}\left(z_{1}, C z_{2}\right)\right)\right)
$$

for all primes $p$.

Proof. The proof is similar to that of Lemma 1.1 of [14].

\section{Statement of the main theorem}

In this section, we state the main theorem and we give numerical examples.

Let $\kappa$ be a positive integer and

$$
f(z)=\sum_{n=1}^{\infty} a_{f}(n) q^{n} \in S_{2 \kappa+1}\left(\Gamma_{0}(D), \chi\right)
$$

be a normalized Hecke eigenform. For $\mathfrak{c} \in J_{K}^{D}$, let $F_{\mathfrak{c}} \in S_{2 \kappa+2}\left(\Gamma_{K}^{(2)}[\mathfrak{c}], \operatorname{det}^{-\kappa-1}\right)$ be the hermitian Maass lift of $f$ defined in the previous section. Let $C=N(\mathfrak{c}) \in \mathbb{Q}>0$ be the ideal norm of $\mathfrak{c}$. For each normalized Hecke eigenform

$$
g(z)=\sum_{n=1}^{\infty} a_{g}(n) q^{n} \in S_{2 \kappa+2}\left(\mathrm{SL}_{2}(\mathbb{Z})\right),
$$

we set $g_{C}(z)=g(z / C)$. Note that $g_{C} \in S_{2 \kappa+2}\left(d(C)^{-1} \mathrm{SL}_{2}(\mathbb{Z}) d(C)\right)$. We consider the period integral $\left\langle\left. F_{\mathfrak{c}}\right|_{\mathfrak{H} \times \mathfrak{H}}, g \times g_{C}\right\rangle$ given by

$$
\begin{aligned}
& \left\langle\left. F_{\mathfrak{c}}\right|_{\mathfrak{H} \times \mathfrak{H}}, g \times g_{C}\right\rangle \\
& =\int_{d(C)^{-1} \mathrm{SL}_{2}(\mathbb{Z}) d(C) \backslash \mathfrak{H}} \int_{\mathrm{SL}_{2}(\mathbb{Z}) \backslash \mathfrak{H}} F_{\mathfrak{c}}\left(\left(\begin{array}{cc}
z_{1} & 0 \\
0 & z_{2}
\end{array}\right)\right) \overline{g\left(z_{1}\right) g_{C}\left(z_{2}\right)} y_{1}^{2 \kappa} y_{2}^{2 \kappa} d z_{1} d z_{2} .
\end{aligned}
$$

Define the Petersson norms of $g$ and $g_{C}$ by

$$
\langle g, g\rangle=\int_{\mathrm{SL}_{2}(\mathbb{Z}) \backslash \mathfrak{H}}|g(z)|^{2} y^{2 \kappa} d z, \quad\left\langle g_{C}, g_{C}\right\rangle=\int_{d(C)^{-1} \mathrm{SL}_{2}(\mathbb{Z}) d(C) \backslash \mathfrak{H}}\left|g_{C}(z)\right|^{2} y^{2 \kappa} d z .
$$

By Lemma 2.2, we get the following lemma.

Lemma 3.1. The map

$$
J_{K}^{D} \ni \mathfrak{c} \mapsto \frac{\left\langle\left. F_{\mathfrak{c}}\right|_{\mathfrak{H} \times \mathfrak{H}}, g \times g_{C}\right\rangle}{\langle g, g\rangle\left\langle g_{C}, g_{C}\right\rangle} \in \mathbb{C}
$$

factors through the ideal class group $\mathrm{Cl}_{K}$.

For $p \notin Q_{D}$, we define the Satake parameter $\left\{\alpha_{f, p}, \chi(p) \alpha_{f, p}^{-1}\right\}$ of $f$ at $p$ by

$$
1-a_{f}(p) X+\chi(p) p^{2 \kappa} X^{2}=\left(1-p^{\kappa} \alpha_{f, p} X\right)\left(1-p^{\kappa} \chi(p) \alpha_{f, p}^{-1} X\right) .
$$

For $p \in Q_{D}$, we put $\alpha_{f, p}=p^{-\kappa} a_{f}(p)$. For each prime $p$, we define the Satake parameter $\left\{\alpha_{g, p}, \alpha_{g, p}^{-1}\right\}$ of $g$ at $p$ by

$$
1-a_{g}(p) X+p^{2 \kappa+1} X^{2}=\left(1-p^{\kappa+1 / 2} \alpha_{g, p} X\right)\left(1-p^{\kappa+1 / 2} \alpha_{g, p}^{-1} X\right) .
$$

The Ramanujan conjecture proved by Deligne states that $\left|\alpha_{f, p}\right|=\left|\alpha_{g, p}\right|=1$ for all $p$. In particular, we have $\left|D^{-k} a_{f}(D)\right|=1$ and $a_{g}(n) \in \mathbb{R}$ for all $n \in \mathbb{Z}_{>0}$. We put

$$
A_{p}=\left\{\begin{array}{cc}
\left(\begin{array}{cc}
\alpha_{f, p} & 0 \\
0 & \chi(p) \alpha_{f, p}^{-1}
\end{array}\right) & \text { if } p \nmid D, \\
\alpha_{f, p} & \text { if } p \mid D,
\end{array} \text { and } \quad B_{p}=\left(\begin{array}{cc}
\alpha_{g, p} & 0 \\
0 & \alpha_{g, p}^{-1}
\end{array}\right) .\right.
$$


Define the $L$-functions $L(s, f \times g)$ and $L(s, f \times g \times \chi)$ by Euler products

$$
\begin{gathered}
L(s, f \times g)=\prod_{p \nmid D} \operatorname{det}\left(\mathbf{1}_{4}-A_{p} \otimes B_{p} \cdot p^{-s}\right)^{-1} \times \prod_{p \mid D} \operatorname{det}\left(\mathbf{1}_{2}-A_{p} \otimes B_{p} \cdot p^{-s}\right)^{-1}, \\
L(s, f \times g \times \chi)=\prod_{p \nmid D} \operatorname{det}\left(\mathbf{1}_{4}-A_{p}^{-1} \otimes B_{p} \cdot p^{-s}\right)^{-1} \times \prod_{p \mid D} \operatorname{det}\left(\mathbf{1}_{2}-A_{p}^{-1} \otimes B_{p} \cdot p^{-s}\right)^{-1} .
\end{gathered}
$$

for $\operatorname{Re}(s) \gg 0$. Note that $L(s, f \times g \times \chi)=\overline{L(\bar{s}, f \times g)}$ by the Ramanujan conjecture. We also define

$$
\mathcal{D}(s, f, g)=\sum_{n=1}^{\infty} n^{-s} a_{f}(n) a_{g}(n)=\sum_{n=1}^{\infty} n^{-s} a_{f}(n) \overline{a_{g}(n)} .
$$

Then, by [24] Lemma 1, we have

$$
\mathcal{D}(s+2 \kappa+1, f, g)=L(2 s+1, \chi)^{-1} L(s+1 / 2, f \times g),
$$

where $L(s, \chi)$ is the Dirichlet $L$-function associated to $\chi$.

Let $\Lambda(s, f \times g)$ and $\Lambda(s, f \times g \times \chi)$ be the completed $L$-functions given by

$$
\begin{aligned}
\Lambda(s, f \times g) & =\Gamma_{\mathbb{C}}(s+1 / 2) \Gamma_{\mathbb{C}}(s+2 \kappa+1 / 2) L(s, f \times g), \\
\Lambda(s, f \times g \times \chi) & =\Gamma_{\mathbb{C}}(s+1 / 2) \Gamma_{\mathbb{C}}(s+2 \kappa+1 / 2) L(s, f \times g \times \chi) .
\end{aligned}
$$

By [18] Theorem 19.14, they have meromorphic continuations to the whole $s$-plane and satisfy the functional equation

$$
\Lambda(s, f \times g)=\varepsilon(s, f \times g) \Lambda(1-s, f \times g \times \chi) .
$$

Here, $\varepsilon(s, f \times g)$ is the $\varepsilon$-factor which will be defined in the next section.

Our main result is as follows.

Theorem 3.2. The identity

$$
L\left(\frac{1}{2}, f \times g\right)=\frac{L(1, \chi)(4 \pi)^{2 \kappa+1}}{a_{f}(D)(2 \kappa) !} \cdot \frac{1}{h_{K}} \sum_{[\mathfrak{c}] \in C l_{K}} \frac{\left\langle\left. F_{\mathfrak{c}}\right|_{\mathfrak{H} \times \mathfrak{H}}, g \times g_{C}\right\rangle}{\left\langle g_{C}, g_{C}\right\rangle}
$$

holds.

Remark 3.3. A special case of a result of Shimura ([24] Theorem 3) asserts that

$$
\pi^{-(2 \kappa+1)}\langle g, g\rangle^{-1} \mathcal{D}(2 \kappa+1, f, g) \in \mathbb{Q}(f) \mathbb{Q}(g) \subset \overline{\mathbb{Q}}
$$

and for all $\sigma \in \operatorname{Aut}(\mathbb{C})$, one has

$$
\left[\pi^{-(2 \kappa+1)}\langle g, g\rangle^{-1} \mathcal{D}(2 \kappa+1, f, g)\right]^{\sigma}=\pi^{-(2 \kappa+1)}\left\langle g^{\sigma}, g^{\sigma}\right\rangle^{-1} \mathcal{D}\left(2 \kappa+1, f^{\sigma}, g^{\sigma}\right) .
$$

Here $\mathbb{Q}(f)($ resp. $\mathbb{Q}(g))$ is the algebraic number field generated by the coefficients $\left\{a_{f}(n)\right\}$ of $f$ (resp. $\left\{a_{g}(n)\right\}$ of $\left.g\right)$. Note that in Shimura's paper, one takes the measure on $\mathrm{SL}_{2}(\mathbb{Z}) \backslash \mathfrak{H}$ so that $\operatorname{vol}\left(\mathrm{SL}_{2}(\mathbb{Z}) \backslash \mathfrak{H}\right)=1$. Since

and for all $\sigma \in \operatorname{Aut}(\mathbb{C})$, one has

$$
\frac{\left\langle\left. F_{\mathfrak{c}}\right|_{\mathfrak{H} \times \mathfrak{H}}, g \times g_{C}\right\rangle}{\left\langle g_{C}, g_{C}\right\rangle\langle g, g\rangle} \in \mathbb{Q}(f) \mathbb{Q}(g)
$$

$$
\left[\frac{\left\langle\left. F_{\mathfrak{c}}\right|_{\mathfrak{H} \times \mathfrak{H}}, g \times g_{C}\right\rangle}{\left\langle g_{C}, g_{C}\right\rangle\langle g, g\rangle}\right]^{\sigma}=\frac{\left\langle\left. F_{\mathfrak{c}}^{\sigma}\right|_{\mathfrak{H} \times \mathfrak{H}}, g^{\sigma} \times g_{C}^{\sigma}\right\rangle}{\left\langle g_{C}^{\sigma}, g_{C}^{\sigma}\right\rangle\left\langle g^{\sigma}, g^{\sigma}\right\rangle},
$$

we find that Theorem 3.2 is compatible with this result.

Remark 3.4. Let $g, g_{1}, g_{2} \in S_{2 \kappa+2}\left(\mathrm{SL}_{2}(\mathbb{Z})\right)$ be normalized Hecke eigenforms. If $g_{1} \neq g_{2}$, then

$$
\left\langle\left. F_{\mathfrak{c}}\right|_{\mathfrak{H} \times \mathfrak{H}}, g_{1} \times\left(g_{2}\right)_{C}\right\rangle=0
$$

by Lemma 2.3 and the multiplicity one theorem. On the other hand, by Lemma 2.1, we find that

$$
\left\langle\left. F_{\mathfrak{c}}\right|_{\mathfrak{H} \times \mathfrak{H}}, g \times g_{C}\right\rangle \in \sqrt{-1} \mathbb{R} \text {. }
$$


Example 3.5. We discuss the case $D=3$ and $\kappa=5$. Then the class number of $K=\mathbb{Q}(\sqrt{-3})$ is $h_{K}=1$. Let $f \in S_{11}\left(\Gamma_{0}(3), \chi\right)$ be the Hecke eigenform such that

$$
f(z)=q+a q^{2}+(9 a-27) q^{3}+304 q^{4}-106 a q^{5}+(-27 a-6480) q^{6}+17234 q^{7}+O\left(q^{8}\right),
$$

with $a=12 \sqrt{-5}$. Here we have used MAGMA [2]. Let $g \in S_{12}\left(\mathrm{SL}_{2}(\mathbb{Z})\right)$ be the normalized Hecke eigenform. We put $\mathcal{A}=\left\{\alpha \in \sqrt{-D}^{-1} \mathfrak{o} \mid N(\alpha)<1\right\}$. Then we have

$$
\frac{\left\langle\left. F_{\mathfrak{o}}\right|_{\mathfrak{H} \times \mathfrak{H}}, g \times g\right\rangle}{\langle g, g\rangle^{2}}=\sum_{\alpha \in \mathcal{A}} \alpha_{F}\left(D \operatorname{det}\left(\begin{array}{cc}
1 & \alpha \\
\bar{\alpha} & 1
\end{array}\right)\right) .
$$

It is easy to see that

$$
\mathcal{A}=\left\{\frac{a}{\sqrt{-D}}+\frac{(1+\sqrt{-D}) b}{2 \sqrt{-D}} \mid(a, b) \in\{(0, \pm 1),(1,-1),( \pm 1,0),(0,0),(-1,1)\}\right\} .
$$

Since $\underline{\chi}_{3}(-3)=-1$, we have

$$
\frac{\left\langle\left. F_{\mathfrak{o}}\right|_{\mathfrak{H} \times \mathfrak{H}}, g \times g\right\rangle}{\langle g, g\rangle^{2}}=\alpha_{F}(3)+6 \alpha_{F}(2)=\left(a_{f}(3)+\underline{\chi}_{3}(-3) \overline{a_{f}(3)}\right)+6 a_{f}(2)=24 a .
$$

On the other hand, by the Dirichlet class number formula, we have

$$
L(1, \chi)=\frac{2 \pi}{w_{K} \sqrt{D}} h_{K}=\frac{2 \pi}{6 \sqrt{3}}=\frac{\pi}{3 \sqrt{3}},
$$

where $w_{K}$ is the number of roots of unity contained in $K$.

Next, by using Dokchitser's program [4], we have computed

$$
\begin{aligned}
&\langle g, g\rangle=0.00000103536205680432092234781681222516459322490796 \cdots, \\
& L(1 / 2 . f \times g)=(0.56063396812989843884129232097782681505979754470872 \cdots) \\
&-(0.06268078316169517780418095155244859459590450529843 \cdots) \sqrt{-1}
\end{aligned}
$$

Therefore we have

$$
\begin{aligned}
& \frac{L(1 / 2, f \times g) a_{f}(D)(2 \kappa) !}{L(1, \chi)(4 \pi)^{2 \kappa+1}\langle g, g\rangle} \\
& =(643.987577519939432565842016594607555806898087568119408590018 \cdots) \sqrt{-1}
\end{aligned}
$$

This numerical value coincides with $24 a=24 \times 12 \sqrt{-5}$.

Example 3.6. We discuss the case $D=15$ and $\kappa=5$. Then the class number of $K=\mathbb{Q}(\sqrt{-15})$ is $h_{K}=2$. Let $\mathfrak{c}=\mathfrak{p}=(17,(-11+\sqrt{-15}) / 2)$. This is a prime ideal above $p=N(\mathfrak{p})=17$. The set $\{\mathfrak{o}, \mathfrak{p}\} \subset J_{K}^{D}$ gives a complete system of representatives of $C l_{K}$. By using MAGMA [2], we find that there is a normalized Hecke eigenform $f \in S_{11}\left(\Gamma_{0}(15), \chi\right)$ given by

$$
\begin{aligned}
f(z) & =q+(50.905 \cdots) q^{2}+((190.983 \cdots)+(150.247 \cdots) \sqrt{-1}) q^{3} \\
& +(1567.405 \cdots) q^{4}+((-553.573 \cdots)+(3075.578 \cdots) \sqrt{-1}) q^{5}+O\left(q^{6}\right) .
\end{aligned}
$$

This satisfies $[\mathbb{Q}(f): \mathbb{Q}]=16$. Let $g \in S_{12}\left(\mathrm{SL}_{2}(\mathbb{Z})\right)$ be the normalized Hecke eigenform. Then

$$
\begin{aligned}
a_{f}(15) & =-(567822.22270986528973314404962089700180196520977555 \cdots) \\
& +(504210.58499582110937058027520540332841350431820609 \cdots \sqrt{-1})
\end{aligned}
$$


and

$$
\begin{aligned}
& \frac{\left\langle\left. F_{\mathfrak{o}}\right|_{\mathfrak{H} \times \mathfrak{H}}, g \times g\right\rangle}{\langle g, g\rangle^{2}} \\
& =-(1835447.760700852448788571784258603965801252414612281062 \cdots) \sqrt{-1}, \\
& \frac{\left\langle\left. F_{\mathfrak{p}}\right|_{\mathfrak{H} \times \mathfrak{H}}, g \times g_{p}\right\rangle}{\langle g, g\rangle\left\langle g_{p}, g_{p}\right\rangle} \\
& =(3003750.957757391660006591292629544475281083622485436259 \cdots) \sqrt{-1}
\end{aligned}
$$

Next, by using Dokchitser's program [4], we have computed

$$
\begin{aligned}
L(1, \chi) & =1.6223114703894447587811843081191756199820036252694 \cdots \\
L(1 / 2, f \times g) & =(0.2917406142511291654221274607868148913729458549395 \cdots) \\
& -(0.3285468591267089836525964409676435446168105516850 \cdots) \sqrt{-1}
\end{aligned}
$$

Therefore the numerical values of

$$
\frac{L(1 / 2, f \times g) a_{f}(D)(2 \kappa) !}{L(1, \chi)(4 \pi)^{2 \kappa+1}\langle g, g\rangle} \text { and } \frac{1}{h_{K}}\left(\frac{\left\langle\left. F\right|_{\mathfrak{H} \times \mathfrak{H}}, g \times g\right\rangle}{\langle g, g\rangle^{2}}+\frac{\left\langle\left. F_{\mathfrak{p}}\right|_{\mathfrak{H} \times \mathfrak{H}}, g \times g_{p}\right\rangle}{\langle g, g\rangle\left\langle g_{p}, g_{p}\right\rangle}\right)
$$

are both

$$
(584151.598528269605609009754185470254739915603936577598344426 \cdots) \sqrt{-1} .
$$

\section{Automorphic forms ON $\mathrm{GL}_{2}$}

In this section, we recall the theory of automorphic forms on $\mathrm{GL}_{2}$.

4.1. Automorphic forms and representations. Let $f$ be an automorphic form on $\mathrm{GL}_{2}\left(\mathbb{A}_{\mathbb{Q}}\right)$ and $\chi$ be a character of $\mathbb{A}_{\mathbb{Q}}^{\times} / \mathbb{Q}^{\times}$. We say that $f$ has a central character $\chi$ if $f$ satisfies

$$
f(a g)=\chi(a) f(g)
$$

for $a \in \mathbb{A}_{\mathbb{Q}}^{\times}$and $g \in \mathrm{GL}_{2}\left(\mathbb{A}_{\mathbb{Q}}\right)$. Let $\psi=\psi_{0}$ be the standard character of $\mathbb{A}_{\mathbb{Q}}$. For $\xi \in \mathbb{Q}$, we define the $\xi$-th Fourier coefficient $W_{f, \xi}$ of $f$ by

$$
W_{f, \xi}(g)=\int_{\mathbb{Q} \backslash \mathbb{A}_{\mathbb{Q}}} f(n(x) g) \overline{\psi(\xi x)} d x .
$$

Let $f=\sum_{n>0} a_{f}(n) q^{n} \in S_{2 \kappa+1}\left(\Gamma_{0}(D), \chi\right)$ and $g=\sum_{n>0} a_{g}(n) q^{n} \in S_{2 \kappa+2}\left(\mathrm{SL}_{2}(\mathbb{Z})\right)$ be normalized Hecke eigenforms. The automorphic form $f$ gives a cusp form $\mathbf{f}$ on $\mathrm{GL}_{2}\left(\mathbb{A}_{\mathbb{Q}}\right)$ by the formula

$$
\mathbf{f}(\alpha)=\underline{\chi}(d)\left(f \mid \alpha_{\infty}\right)(\sqrt{-1})
$$

for $\alpha=\gamma \alpha_{\infty} k \in \mathrm{GL}_{2}\left(\mathbb{A}_{\mathbb{Q}}\right)$ with $\gamma \in \mathrm{GL}_{2}(\mathbb{Q}), \alpha_{\infty} \in \mathrm{GL}_{2}(\mathbb{R})^{+}$and

$$
k=\left(\begin{array}{ll}
a & b \\
c & d
\end{array}\right) \in \mathbf{K}_{0}(D ; \hat{\mathbb{Z}}), \quad \text { where } \quad \mathbf{K}_{0}(D ; \hat{\mathbb{Z}})=\left\{\left(\begin{array}{ll}
a & b \\
c & d
\end{array}\right) \in \mathrm{GL}_{2}(\hat{\mathbb{Z}}) \mid c \in D \hat{\mathbb{Z}}\right\} .
$$

Note that the central character of $\mathbf{f}$ is $\chi$. The automorphic form $g$ gives a cusp form $\mathbf{g}$ on $\mathrm{GL}_{2}\left(\mathbb{A}_{\mathbb{Q}}\right)$ by the formula

$$
\mathbf{g}(\beta)=\left(g \mid \beta_{\infty}\right)(\sqrt{-1})
$$

for $\beta=\gamma^{\prime} \beta_{\infty} k^{\prime}$ with $\gamma^{\prime} \in \mathrm{GL}_{2}(\mathbb{Q}), \beta_{\infty} \in \mathrm{GL}_{2}(\mathbb{R})^{+}$and $k^{\prime} \in \mathrm{GL}_{2}(\hat{\mathbb{Z}})$. Note that the central character of $\mathbf{g}$ is the trivial character.

Let $\pi_{f} \cong \otimes_{v}^{\prime} \pi_{f, v}$ (resp. $\left.\pi_{g} \cong \otimes_{v}^{\prime} \pi_{g, v}\right)$ be the irreducible cuspidal automorphic representation of $\mathrm{GL}_{2}\left(\mathbb{A}_{\mathbb{Q}}\right)$ generated by $\mathbf{f}$ (resp. $\mathbf{g}$ ). Then the central character of $\pi_{f}$ (resp. $\pi_{g}$ ) is $\chi$ (resp. the 
trivial character). The $\infty$-component $\pi_{f, \infty}$ (resp. $\left.\pi_{g, \infty}\right)$ is the (limit of) discrete series representation of $\mathrm{GL}_{2}(\mathbb{R})$ with minimal weight $\pm(2 \kappa+1)$ (resp. $\pm(2 \kappa+2)$ ). The $p$-component $\pi_{f, p}$ (resp. $\pi_{g, p}$ ) is the principal series representation

$$
\pi_{f, p} \cong \operatorname{Ind}_{\mathbf{B}_{p}}^{\mathbf{G}_{p}}\left(|\cdot|_{p}^{s_{f, p}} \otimes \underline{\chi}_{p}|\cdot|_{p}^{-s_{f, p}}\right), \quad\left(\text { resp. } \pi_{g, p} \cong \operatorname{Ind}_{\mathbf{B}_{p}}^{\mathbf{G}_{p}}\left(|\cdot|_{p}^{s_{g, p}} \otimes|\cdot|_{p}^{-s_{g, p}}\right)\right) .
$$

Here we put $\mathbf{G}_{p}=\mathrm{GL}_{2}\left(\mathbb{Q}_{\mathfrak{p}}\right)$ and we denote $\mathbf{B}_{p}$ the Borel subgroup of $\mathbf{G}_{p}$ consisting all upper triangle matrices, and $s_{f, p}, s_{g, p} \in \mathbb{C}$ satisfy $|p|_{p}^{s_{f, p}}=\alpha_{f, p}$ and $|p|_{p}^{s_{g, p}}=\alpha_{g, p}$. The restriction of $\pi_{f, \infty}$ to $\mathrm{SL}_{2}(\mathbb{R})$ is the direct sum of two irreducible representations $\pi_{f}^{+}$and $\pi_{f}^{-}$, i.e., $\left.\pi_{f, \infty}\right|_{\mathrm{SL}_{2}(\mathbb{R})} \cong \pi_{f}^{+} \oplus \pi_{f}^{-}$. Here, $\pi_{f}^{+}$(resp. $\pi_{f}^{-}$) is the holomorphic (resp. anti-holomorphic) discrete series representation of $\mathrm{SL}_{2}(\mathbb{R})$ with minimal weight $2 \kappa+1$ (resp. $-(2 \kappa+1)$ ). Note that $\mathbf{f} \in \pi_{f}^{+} \otimes\left(\otimes_{p}^{\prime} \pi_{f, p}\right)$.

4.2. The $L$-functions. Let $\pi=\pi_{f} \times \pi_{g}$. Then by [18] $\S 14, \S 17$ and $\S 19$, we can define the $L$-functions and the $\varepsilon$-factor

$$
L(s, \pi)=\prod_{v} L\left(s, \pi_{v}\right), \quad L\left(s, \pi^{\vee}\right)=\prod_{v} L\left(s, \pi_{v}^{\vee}\right), \quad \varepsilon(s, \pi)=\prod_{v} \varepsilon\left(s, \pi_{v}, \psi_{v}\right)
$$

where $\pi^{\vee}$ is the contragredient representation of $\pi$. By [18] Theorem 19.14, we have the functional equation

$$
L(s, \pi)=\varepsilon(s, \pi) L\left(1-s, \pi^{\vee}\right) .
$$

Let $\Lambda(s, f \times g)$ and $\Lambda(s, f \times g \times \chi)$ be the completed $L$-functions defined in Sect. 3 .

Lemma 4.1. We have

$$
\Lambda(s, f \times g)=L(s, \pi), \quad \Lambda(s, f \times g \times \chi)=L\left(s, \pi^{\vee}\right) .
$$

and

$$
\varepsilon(s, \pi)=-D^{1-2 s+2 \kappa} a_{f}(D)^{-2} .
$$

Proof. Since $\prod_{p<\infty} \underline{\chi}_{p}(-1)=\underline{\chi}(-1) \underline{\chi}_{\infty}(-1)^{-1}=-1$, it is enough to show the equations

$$
\begin{aligned}
L\left(s, \pi_{p}\right) & =\operatorname{det}\left(\mathbf{1}_{r}-A_{p} \otimes B_{p} \cdot p^{-s}\right)^{-1}, \\
L\left(s, \pi_{p}^{\vee}\right) & =\operatorname{det}\left(\mathbf{1}_{r}-A_{p}^{-1} \otimes B_{p} \cdot p^{-s}\right)^{-1}, \\
\varepsilon\left(s, \pi_{p}, \psi_{p}\right) & =\underline{\chi}_{p}(-1)\left(p^{d}\right)^{1-2 s+2 \kappa} a_{f}\left(p^{d}\right)^{-2}
\end{aligned}
$$

for $p<\infty$ with $d=\operatorname{ord}_{p}(D)$ and

$$
r= \begin{cases}4 & \text { if } p \nmid D, \\ 2 & \text { if } p \mid D,\end{cases}
$$

and

$$
\begin{aligned}
L\left(s, \pi_{\infty}\right)=L\left(s, \pi_{\infty}^{\vee}\right) & =\Gamma_{\mathbb{C}}(s+1 / 2) \Gamma_{\mathbb{C}}(s+2 \kappa+1 / 2), \\
\varepsilon\left(s, \pi_{\infty}, \psi_{\infty}\right) & =1 .
\end{aligned}
$$

For $v=p<\infty$, by [18] Theorem 15.1, we have

$$
\begin{aligned}
\varepsilon\left(s, \pi_{p}, \psi_{p}\right)= & \varepsilon\left(s, \pi_{f, p} \otimes|\cdot|_{p}^{s_{g, p}}, \psi_{p}\right) \varepsilon\left(s,\left.\pi_{f, p} \otimes|\cdot|\right|_{p} ^{-s_{g, p}}, \psi_{p}\right) \\
= & \varepsilon\left(s,|\cdot|_{p}^{s_{f, p}+s_{g, p}}, \psi_{p}\right) \varepsilon\left(s,|\cdot|_{p}^{s_{f, p}-s_{g, p}}, \psi_{p}\right) \\
& \times \varepsilon\left(s, \underline{\chi}_{p}|\cdot|_{p}^{-s_{f, p}+s_{g, p}}, \psi_{p}\right) \varepsilon\left(s, \underline{\chi}_{p}|\cdot|_{p}^{-s_{f, p}-s_{g, p}}, \psi_{p}\right) \\
= & \varepsilon\left(s-s_{f, p}+s_{g, p}, \underline{\chi}_{p}, \psi_{p}\right) \varepsilon\left(s-s_{f, p}-s_{g, p}, \underline{\chi}_{p}, \psi_{p}\right) .
\end{aligned}
$$

If $p \nmid D$, then the character $\underline{\chi}_{p}$ is unramified. So we have

$$
\varepsilon\left(s, \pi_{p}, \psi_{p}\right)=1=\underline{\chi}_{p}(-1)\left(p^{d}\right)^{1-2 s+2 \kappa} a_{f}\left(p^{d}\right)^{-2} .
$$


If $p \mid D$, then $d=\operatorname{ord}_{p}(D)>0$. Let $U_{p}^{(0)}=\mathbb{Z}_{p}^{\times}$and $U_{p}^{(n)}=1+p^{n} \mathbb{Z}_{p}$ for $n \geq 1$. We have $\underline{\chi}_{p} \mid U_{p}^{(d)}=1$ and $\underline{\chi}_{p} \mid U_{p}^{(d-1)} \neq 1$. Then it is well-known that

$$
\varepsilon\left(s, \underline{\chi}_{p}, \psi_{p}\right)=p^{d(1 / 2-s)} \varepsilon\left(1 / 2, \underline{\chi}_{p}, \psi_{p}\right)
$$

(see e.g., [3] 23.5 Theorem). So we have

$$
\varepsilon\left(s, \pi_{p}, \psi_{p}\right)=p^{d(1-2 s)} p^{2 d s_{f, p}} \varepsilon\left(1 / 2, \underline{\chi}_{p}, \psi_{p}\right)^{2} .
$$

It is well-know that $\varepsilon\left(1 / 2, \underline{\chi}_{p}, \psi_{p}\right)^{2}=\underline{\chi}_{p}(-1)$ (see e.g., [3] 23.4 Corollary 2). On the other hand, we have $p^{2 d s_{f, p}}=\alpha_{f, p}^{-2 d}=\left(p^{-\kappa} a_{f}(p)\right)^{-2 d}=\left(p^{d}\right)^{2 \kappa} a_{f}\left(p^{d}\right)^{-2}$. This gives the desired formula for $\varepsilon\left(s, \pi_{p}, \psi_{p}\right)$. By calculations similar to that of $\varepsilon\left(s, \pi_{p}, \psi_{p}\right)$, we have the desired formulas for $L\left(s, \pi_{p}\right)$ and $L\left(s, \pi_{p}^{\vee}\right)$.

Next, we assume that $v=\infty$. Let $W_{\mathbb{R}}=\mathbb{C}^{\times} \cup j \mathbb{C}^{\times}$be the Weil group of $\mathbb{R}$ with $j^{2}=-1$ and $j z j^{-1}=\bar{z}$ for $z \in \mathbb{C}^{\times}$. Let $\rho_{f, \infty}\left(\operatorname{resp} \rho_{g, \infty}\right)$ be the two dimensional representation of $W_{\mathbb{R}}$ which corresponds to $\pi_{f, \infty}$ (resp. $\pi_{g, \infty}$ ) by the local Langlands correspondence. Then by [18] Proposition 17.3, we have

$$
\begin{gathered}
L\left(s, \pi_{\infty}\right)=L\left(s, \rho_{f, \infty} \otimes \rho_{g, \infty}\right), \quad L\left(s, \pi_{\infty}^{\vee}\right)=L\left(s, \rho_{f, \infty}^{\vee} \otimes \rho_{g, \infty}^{\vee}\right), \\
\varepsilon\left(s, \pi_{\infty}, \psi_{\infty}\right)=\varepsilon\left(s, \rho_{f, \infty} \otimes \rho_{g, \infty}, \psi_{\infty}\right) .
\end{gathered}
$$

For $n \in \mathbb{Z}$, we define the two dimensional representation $\rho_{n}$ of $W_{\mathbb{R}}$ by

$$
\begin{gathered}
\rho_{n}: W_{\mathbb{R}}=\mathbb{C}^{\times} \cup j \mathbb{C}^{\times} \rightarrow \mathrm{GL}_{2}(\mathbb{C}), \\
r e^{\sqrt{-1} \theta} \mapsto\left(\begin{array}{cc}
e^{n \sqrt{-1} \theta} & 0 \\
0 & e^{-n \sqrt{-1} \theta}
\end{array}\right), \quad j \mapsto\left(\begin{array}{cc}
0 & (-1)^{n} \\
1 & 0
\end{array}\right) .
\end{gathered}
$$

Then we have $\rho_{-n} \cong \rho_{n}$ for $n \in \mathbb{Z}$. Hence $\rho_{n}$ is a self-dual representation. Moreover, for $n_{1}, n_{2} \in \mathbb{Z}$, we have $\rho_{n_{1}} \otimes \rho_{n_{2}} \cong \rho_{n_{1}+n_{2}} \oplus \rho_{n_{1}-n_{2}}$. For $n \in \mathbb{Z}_{>0}$, the $L$-function and the $\varepsilon$-factor associated to $\rho_{n}$ are given by

$$
L\left(s, \rho_{n}\right)=\Gamma_{\mathbb{C}}(s+n / 2), \quad \varepsilon\left(s, \rho_{n}, \psi_{\infty}\right)=\sqrt{-1}^{n+1} .
$$

See [25]. It is well-known that $\rho_{f, \infty} \cong \rho_{2 \kappa}$ and $\rho_{g, \infty} \cong \rho_{2 \kappa+1}$. So we have

$$
\begin{gathered}
L\left(s, \pi_{\infty}\right)=L\left(s, \pi_{\infty}^{\vee}\right)=\Gamma_{\mathbb{C}}(s+2 \kappa+1 / 2) \Gamma_{\mathbb{C}}(s+1 / 2), \\
\varepsilon\left(s, \pi_{\infty}, \psi_{\infty}\right)=\sqrt{-1}^{(4 \kappa+2)+2}=1 .
\end{gathered}
$$

This completes the proof.

We put $\varepsilon(s, f \times g)=\varepsilon(s, \pi)$. Then we have the functional equation noted in Sect. 3. In particular, this functional equation and the Ramanujan conjecture imply that

$$
a_{f}(D) D^{-\kappa} \Lambda(1 / 2, f \times g)=-\left(a_{f}(D) D^{-\kappa}\right)^{-1} \Lambda(1 / 2, f \times g \times \chi)=-\overline{a_{f}(D) D^{-\kappa} \Lambda(1 / 2, f \times g)} .
$$

So we find that

$$
a_{f}(D) L(1 / 2, f \times g) \in \sqrt{-1} \mathbb{R} .
$$

4.3. The Whittaker function on $\mathrm{GL}_{2}$. Let $\psi=\psi_{0}$ be the standard character of $\mathbb{A}_{\mathbb{Q}}$. The Whittaker function $W_{\mathbf{f}}\left(\right.$ resp. $W_{\mathbf{g}}$ ) of $\mathbf{f}$ (resp. $\mathbf{g}$ ) is defined by

$$
W_{\mathbf{f}}(\alpha)=\int_{\mathbb{Q} \backslash \mathbb{A}_{\mathbb{Q}}} \mathbf{f}(n(x) \alpha) \overline{\psi(x)} d x \quad\left(\text { resp. } \quad W_{\mathbf{g}}(\alpha)=\int_{\mathbb{Q} \backslash \mathbb{A}_{\mathbb{Q}}} \mathbf{g}(n(x) \alpha) \overline{\psi(x)} d x\right) .
$$


Note that $W_{\mathbf{f}, \xi}(\alpha)=W_{\mathbf{f}}(a(\xi) \alpha)$ and $W_{\mathbf{g}, \xi}(\alpha)=W_{\mathbf{g}}(a(\xi) \alpha)$ for $\xi \in \mathbb{Q}^{\times}$. The function $W_{\mathbf{f}}$ (resp. $W_{\mathbf{g}}$ ) has a product expansion $W_{\mathbf{f}}=\prod_{v} W_{\mathbf{f}, v}\left(\right.$ resp. $\left.W_{\mathbf{g}}=\prod_{v} W_{\mathbf{g}, v}\right)$, where

$$
\begin{aligned}
& W_{\mathbf{f}, \infty}\left(n(y) a(x) k_{\theta}\right)= \begin{cases}e^{2 \pi \sqrt{-1} y} x^{\kappa+(1 / 2)} e^{-2 \pi x} e^{\sqrt{-1}(2 \kappa+1) \theta} & \text { if } x>0, \\
0 & \text { if } x<0,\end{cases} \\
& W_{\mathbf{g}, \infty}\left(n(y) a(x) k_{\theta}\right)= \begin{cases}e^{2 \pi \sqrt{-1} y} x^{\kappa+1} e^{-2 \pi x} e^{\sqrt{-1}(2 \kappa+2) \theta} & \text { if } x>0, \\
0 & \text { if } x<0 .\end{cases}
\end{aligned}
$$

For $p \nmid D$, the function $W_{\mathbf{f}, p}$ satisfies $W_{\mathbf{f}, p}(\alpha k)=W_{\mathbf{f}, p}(\alpha)$ for all $\alpha \in \mathrm{GL}_{2}\left(\mathbb{Q}_{p}\right)$ and $k \in \mathrm{GL}_{2}\left(\mathbb{Z}_{p}\right)$. For $p \mid D$, the function $W_{\mathbf{f}, p}$ satisfies $W_{\mathbf{f}, p}(\alpha k)=\underline{\chi}_{p}(k) W_{\mathbf{f}, p}(\alpha)$ for all $\alpha \in \mathrm{GL}_{2}\left(\mathbb{Q}_{p}\right)$ and $k \in \mathbf{K}_{0}\left(D ; \mathbb{Z}_{p}\right)$. Here, we define

$$
\underline{\chi}_{p}(k)=\underline{\chi}_{p}(d), \quad \text { for } \quad k=\left(\begin{array}{ll}
a & b \\
c & d
\end{array}\right) \in \mathbf{K}_{0}\left(D ; \mathbb{Z}_{p}\right) .
$$

For each prime $p$, the function $W_{\mathbf{g}, p}$ satisfies $W_{\mathbf{g}, p}(\alpha k)=W_{\mathbf{g}, p}(\alpha)$ for all $\alpha \in \mathrm{GL}_{2}\left(\mathbb{Q}_{p}\right)$ and $k \in \mathrm{GL}_{2}\left(\mathbb{Z}_{p}\right)$.

The following lemma is a reformulation of Lemma 6.3 of [16] in terms of the Fourier coefficients of $f$ and $g$. Here, we put $a_{f}(x)=0$ and $a_{g}(x)=0$ unless $x \in \mathbb{Z}_{>0}$. For $x=p^{n} u \in \mathbb{Q}_{p}^{\times}$with $u \in \mathbb{Z}_{p}^{\times}$, we set $x_{p}=p^{n}$.

Lemma 4.2. We put $d=\operatorname{ord}_{p}(D), \zeta_{8}=\exp (\pi \sqrt{-1} / 4)$,

$$
\varepsilon= \begin{cases}1 & \text { if } \underline{\chi}_{p}(-1)=1, \\ \sqrt{-1} & \text { if } \underline{\chi}_{p}(-1)=-1,\end{cases}
$$

and

$$
w=\left(\begin{array}{cc}
0 & -1 \\
1 & 0
\end{array}\right), \quad k_{1}=\left(\begin{array}{cc}
1 & 0 \\
p^{d-1} & 1
\end{array}\right), \quad k_{2}=\left(\begin{array}{cc}
1 & 0 \\
p^{d-2} & 1
\end{array}\right) .
$$

(1) For each prime p, we have

$$
W_{\mathbf{g}, p}(a(x))=|x|_{p}^{\kappa+1} a_{g}\left(x_{p}\right) .
$$

(2) For $p \nmid D$, we have

$$
W_{\mathbf{f}, p}(a(x))=|x|_{p}^{\kappa+1 / 2} a_{f}\left(x_{p}\right) .
$$

(3) For $p \mid D$, we have

$$
W_{\mathbf{f}, p}(a(x) w)=\underline{\chi}_{p}\left(-D_{p} x\right) \varepsilon^{-1} p^{-d / 2} a_{f}\left(D_{p}\right)^{-1}|x|_{p}^{\kappa+1 / 2} \overline{a_{f}\left((D x)_{p}\right)} .
$$

(4) For $p=2 \mid D$, we have

$$
W_{\mathbf{f}, p}\left(a(x) k_{1}\right)= \begin{cases}|x|_{2}^{\kappa+1 / 2} a_{f}(2)^{-1} & \text { if } \operatorname{ord}_{2}(x)=-1, \\ 0 & \text { otherwise. }\end{cases}
$$

(5) For $p=2, d=3$ and $x=2^{n} u$ with $u \in \mathbb{Z}_{2}^{\times}$, we have

$$
W_{\mathbf{f}, p}\left(a(x) k_{2}\right)= \begin{cases}2^{-1 / 2} \underline{\chi}_{p}(u) \varepsilon \zeta_{8}^{-u}|x|_{2}^{\kappa+1 / 2} a_{f}(2)^{-2} & \text { if } \operatorname{ord}_{2}(x)=-2, \\ 0 & \text { otherwise. }\end{cases}
$$

\section{WeIL REPRESENTATIONS AND THETA LIFTS}

In this section, we recall the theory of Weil representations and theta lifts. 
5.1. Quadratic spaces. Let $F$ be a field of characteristic not 2 and $V$ a quadratic space over $F$. Namely, $V$ is a vector space over $F$ of dimension $m$ equipped with a non-degenerate symmetric bilinear form $($,$) . We assume that m$ is even. Let $Q$ denote the associated quadratic form on $V$. Then

$$
Q[x]=\frac{1}{2}(x, x)
$$

for $x \in V$. We fix a basis $\left\{v_{1}, \ldots, v_{m}\right\}$ of $V$ and identify $V$ with the space of column vectors $F^{m}$. Define $Q \in \mathrm{GL}_{m}(F)$ by

$$
Q=\left(\left(v_{i}, v_{j}\right)\right)_{i, j}
$$

and let $\operatorname{det}(V)$ denote the image of $\operatorname{det}(Q)$ in $F^{\times} / F^{\times 2}$. Let

$$
\mathrm{GO}(V)=\left\{\left.h \in \mathrm{GL}_{m}\right|^{t} h Q h=\nu(h) Q, \nu(h) \in \mathrm{GL}_{1}\right\}
$$

be the orthogonal similitude group and $\nu: \mathrm{GO}(V) \rightarrow \mathrm{GL}_{1}$ the similitude norm. We put

$$
\operatorname{GSO}(V)=\left\{h \in \mathrm{GO}(V) \mid \operatorname{det}(h)=\nu(h)^{m / 2}\right\} .
$$

Let $\mathrm{O}(V)=\operatorname{ker}(\nu)$ be the orthogonal group and $\mathrm{SO}(V)=\mathrm{O}(V) \cap \mathrm{SL}_{m}$ the special orthogonal group.

5.2. Weil representations. Let $F$ be a local field of characteristic not 2 and $V$ a quadratic space over $F$ of dimension $m$. We assume that $m$ is even. We fix a non-trivial additive character $\psi$ of $F$. For $a \in F^{\times}$, we define a non-trivial additive character $a \psi$ of $F$ by $(a \psi)(x)=\psi(a x)$. We define a quadratic character $\chi_{V}$ of $F^{\times}$by

$$
\chi_{V}(a)=\left((-1)^{m / 2} \operatorname{det}(V), a\right)_{F}
$$

for $a \in F^{\times}$, where $(x, y)_{F}$ is the Hilbert symbol of $F$. We define an 8-th root of unity $\gamma_{V}$ by

$$
\gamma_{V}=\gamma_{F}\left(\operatorname{det}(V), \frac{1}{2} \psi\right) \gamma_{F}\left(\frac{1}{2} \psi\right)^{m} h_{F}(V) .
$$

Here $h_{F}(V)$ is the Hasse invariant of $V$. Note that $\chi_{V}$ and $\gamma_{V}$ depend only on the anisotropic kernel of $V$. To calculate $\gamma_{F}(a, b \psi)$ and $\gamma_{F}(a \psi)$, see [14] A.1.

Let $\omega=\omega_{V, \psi}$ denote the Weil representation of $\mathrm{SL}_{2}(F) \times \mathrm{O}(V)$ on $\mathcal{S}(V)$ with respect to $\psi$. Let $\varphi \in \mathcal{S}(V)$ and $x \in V$. Then,

$$
\begin{aligned}
\omega(1, h) \varphi(x) & =\varphi\left(h^{-1} x\right), \\
\omega(t(a), 1) \varphi(x) & =\chi_{V}(a)|a|_{F}^{m / 2} \varphi(x a), \\
\omega(n(b), 1) \varphi(x) & =\varphi(x) \psi(b Q[x]), \\
\omega(w, 1) \varphi(x) & =\gamma_{V}^{-1} \int_{V} \varphi(y) \psi(-(x, y)) d y
\end{aligned}
$$

for $a \in F^{\times}, b \in F$ and $h \in \mathrm{O}(V)(F)$. Here, $d y$ is the self-dual measure on $V$ with respect to $\psi((x, y))$ given by

$$
d y=|\operatorname{det}(Q)|^{1 / 2} \prod_{j} d y_{j}
$$

where $d y_{j}$ is the self-dual measure on $F$ with respect to $\psi$.

Following [11] $\S 5.1$, we extend the Weil representation $\omega$. We put

$$
R=\mathrm{G}\left(\mathrm{SL}_{2} \times \mathrm{O}(V)\right)=\left\{(g, h) \in \mathrm{GL}_{2} \times \mathrm{GO}(V) \mid \operatorname{det}(g)=\nu(h)\right\} .
$$

For $h \in \mathrm{GO}(V)$ and $\varphi \in \mathcal{S}(V)$, we put

$$
L(h) \varphi(x)=|\nu(h)|_{F}^{-m / 4} \varphi\left(h^{-1} x\right)
$$

for $x \in V$. Then we define the Weil representation $\omega$ of $R(F)$ on $\mathcal{S}(V)$ by

$$
\omega(g, h)=\omega\left(g \cdot d\left(\operatorname{det}(g)^{-1}\right), 1\right) \circ L(h)
$$

for $(g, h) \in R(F)$. 
5.3. Theta functions and theta lifts. Let $V$ be a quadratic space over $\mathbb{Q}$ of even dimension $m$ and $\psi=\psi_{0}$ the standard additive character of $\mathbb{A}_{\mathbb{Q}}$. Let $\omega$ denote the Weil representation of $R\left(\mathbb{A}_{\mathbb{Q}}\right)$ on $\mathcal{S}\left(V\left(\mathbb{A}_{\mathbb{Q}}\right)\right)$ with respect to $\psi$. For $(g, h) \in R\left(\mathbb{A}_{\mathbb{Q}}\right)$ and $\varphi \in \mathcal{S}\left(V\left(\mathbb{A}_{\mathbb{Q}}\right)\right)$, we put

$$
\theta(g, h ; \varphi)=\sum_{x \in V(\mathbb{Q})} \omega(g, h) \varphi(x) .
$$

Then $\theta(g, h ; \varphi)$ is an automorphic form on $R\left(\mathbb{A}_{\mathbb{Q}}\right)$. The function $\theta(g, h ; \varphi)$ is called a theta function.

Let $f$ be a cusp form on $\mathrm{GL}_{2}\left(\mathbb{A}_{\mathbb{Q}}\right)$. For $h \in \mathrm{GO}(V)\left(\mathbb{A}_{\mathbb{Q}}\right)$, choose $g^{\prime} \in \mathrm{GL}_{2}\left(\mathbb{A}_{\mathbb{Q}}\right)$ such that $\operatorname{det}\left(g^{\prime}\right)=\nu(h)$, and put

$$
\theta(h ; f, \varphi)=\int_{\left[\mathrm{SL}_{2}\right]} \theta\left(g g^{\prime}, h ; \varphi\right) f\left(g g^{\prime}\right) d g .
$$

Note that this integral does not depend on the choice of $g^{\prime}$. Then $\theta(f, \varphi)$ is an automorphic form on $\operatorname{GO}(V)\left(\mathbb{A}_{\mathbb{Q}}\right)$. The function $\theta(f, \varphi)$ is called a theta lift. Similarly, we define $\theta\left(f^{\prime}, \varphi\right)$ for a cusp form $f^{\prime}$ on $\mathrm{GO}(V)\left(\mathbb{A}_{\mathbb{Q}}\right)$. More precisely, see [11].

5.4. Change of polarizations. Let $F$ be a field of characteristic not 2 and $V$ a quadratic space over $F$ of even dimension $m$. We assume that the matrix $Q \in \mathrm{GL}_{m}(F)$ associated to $V$ is of the form

$$
Q=\left(\begin{array}{ccc}
0 & 0 & 1 \\
0 & Q_{1} & 0 \\
1 & 0 & 0
\end{array}\right)
$$

for some $Q_{1} \in \mathrm{GL}_{m-2}(F)$. Let $V_{1}=F^{m-2}$ be the quadratic space with bilinear form

$$
(v, w)={ }^{t} v Q_{1} w .
$$

The associated quadratic form on $V_{1}$ is also denoted by $Q_{1}$. For $v \in V_{1}$, we define an element $\ell(v) \in \mathrm{O}(V)(F)$ by

$$
\ell(v)=\left(\begin{array}{ccc}
1 & -{ }^{t} v Q_{1} & -Q_{1}[v] \\
0 & \mathbf{1}_{m-2} & v \\
0 & 0 & 1
\end{array}\right) .
$$

Let $F=\mathbb{Q}_{v}$ (resp. $F=\mathbb{Q}$ ) and $\psi=\psi_{v}\left(\right.$ resp. $\left.\psi=\psi_{0}\right)$ be the standard character of $\mathbb{Q}_{v}$ $\left(\right.$ resp. $\left.\mathbb{A}_{\mathbb{Q}}\right)$. For $\varphi \in \mathcal{S}(V)$ (resp. $\varphi \in \mathcal{S}\left(V\left(\mathbb{A}_{F}\right)\right)$ ), we define the partial Fourier transform by the formula

$$
\hat{\varphi}\left(x_{1} ; y_{1}, y_{2}\right)=\int_{X_{F}} \varphi\left(\begin{array}{c}
z \\
x_{1} \\
y_{1}
\end{array}\right) \psi\left(y_{2} z\right) d z
$$

for $x_{1} \in V_{1}, y_{1}, y_{2} \in \mathbb{Q}_{v}$ and $X_{F}=\mathbb{Q}_{v}$ (resp. $x_{1} \in V_{1}\left(\mathbb{A}_{\mathbb{Q}}\right), y_{1}, y_{2} \in \mathbb{A}_{\mathbb{Q}}$ and $X_{F}=\mathbb{A}_{\mathbb{Q}}$ ). Here $d z$ is the self-dual measure on $X_{F}$ with respect to $\psi$. We define a representation $\hat{\omega}$ of $R\left(X_{F}\right)$ on $\mathcal{S}\left(V_{1}\left(X_{F}\right)\right) \otimes \mathcal{S}\left(X_{F}^{2}\right)$ by

$$
\hat{\omega}(g, h) \hat{\varphi}=(\omega(g, h) \varphi) .
$$

If $\hat{\varphi}=\varphi_{1} \otimes \varphi_{2}$ with $\varphi_{1} \in \mathcal{S}\left(V_{1}\right)\left(X_{F}\right)$ and $\varphi_{2} \in \mathcal{S}\left(X_{F}^{2}\right)$, then

$$
\hat{\omega}(g, 1) \hat{\varphi}\left(x_{1} ; y_{1}, y_{2}\right)=\omega_{V_{1}, \psi}(g, 1) \varphi_{1}\left(x_{1}\right) \cdot \varphi_{2}\left(\left(y_{1}, y_{2}\right) g\right)
$$

for $g \in \mathrm{SL}_{2}\left(X_{F}\right)$. See also [14] $\S 4.2$.

Let $f$ be a cusp form on $\mathrm{GL}_{2}\left(\mathbb{A}_{\mathbb{Q}}\right)$ and $\varphi \in \mathcal{S}\left(V\left(\mathbb{A}_{\mathbb{Q}}\right)\right)$. For $\Xi \in V_{1}(\mathbb{Q})$, define the $\Xi$-th Fourier coefficient $\mathcal{W}_{\Xi}=\mathcal{W}_{\theta(f, \varphi), \Xi}$ of $\theta(f, \varphi)$ by

$$
\mathcal{W}_{\Xi}(h)=\int_{V_{1}(\mathbb{Q}) \backslash V_{1}\left(\mathbb{A}_{\mathbb{Q}}\right)} \theta(\ell(v) h ; f, \varphi) \overline{\psi((\Xi, v))} d v
$$

for $h \in \mathrm{GO}(V)\left(\mathbb{A}_{\mathbb{Q}}\right)$. We need a modification of Lemma 4.2 of [14]. 
Lemma 5.1. (1) If $\Xi \neq 0$, then for $h \in \mathrm{GO}(V)\left(\mathbb{A}_{\mathbb{Q}}\right)$, we have

$$
\mathcal{W}_{\Xi}(h)=\int_{N\left(\mathbb{A}_{\mathbb{Q}}\right) \backslash \mathrm{SL}_{2}\left(\mathbb{A}_{\mathbb{Q}}\right)} \hat{\omega}(g \cdot d(\nu(h)), h) \hat{\varphi}(-\Xi ; 0,1) W_{f,-Q_{1}[\Xi]}(g \cdot d(\nu(h))) d g .
$$

Here, $W_{f, \xi}$ is the $\xi$-th Fourier coefficient of $f$ defined in Sect. 4.

(2) For $h \in \mathrm{GO}(V)\left(\mathbb{A}_{\mathbb{Q}}\right)$, we have

$$
\mathcal{W}_{0}(h)=\int_{\left[\mathrm{SL}_{2}\right]} \sum_{x_{1} \in V_{1}(\mathbb{Q})} \hat{\omega}(g \cdot d(\nu(h)), h) \hat{\varphi}\left(x_{1} ; 0,0\right) f(g \cdot d(\nu(h))) d g .
$$

Proof. For $h \in \mathrm{O}(V)\left(\mathbb{A}_{\mathbb{Q}}\right)$, apply Lemma 4.2 of [14] to $\left.f\right|_{\mathrm{SL}_{2}\left(\mathbb{A}_{\mathbb{Q}}\right)}$. In general, we put $f^{\prime}(g)=$ $f(g \cdot d(\nu(h)))$. Then by [11] Lemma 5.1.7, we have

$$
\begin{aligned}
\theta(\ell(v) h ; f, \varphi) & =\theta\left(\ell(v) ; f^{\prime}, L(h) \varphi\right), \\
\hat{\omega}(g, 1)(L(h) \varphi)^{\wedge}\left(-\Xi, y_{1}, y_{2}\right) & =\hat{\omega}(g \cdot d(\nu(h)), h) \hat{\varphi}\left(-\Xi, y_{1}, y_{2}\right) .
\end{aligned}
$$

On the other hand, we have

$$
W_{f^{\prime}, \xi}(g)=W_{f, \xi}(g \cdot d(\nu(h))) .
$$

These equations imply the formulas.

\section{Theta correspondence for $\left(\mathrm{GU}(2,2), \mathrm{GL}_{2}\right)$}

In this section, we study the hermitian Maass lifting in terms of theta lifts. The main result of this section is Proposition 6.2 which states that the automorphic form Lift $^{(2)}(f)$ on $\mathrm{U}(2,2)\left(\mathbb{A}_{\mathbb{Q}}\right)$ given by the hermitian Maass lifts $\left\{F_{\mathfrak{c}}\right\}$ of $f$ can be written by a certain theta lift via a homomorphism $\phi: \mathrm{GU}(2,2)\left(\mathbb{A}_{\mathbb{Q}}\right) \rightarrow \operatorname{PGSO}(4,2)\left(\mathbb{A}_{\mathbb{Q}}\right)$. This proposition is a key point of the proof of Theorem 3.2.

6.1. Preliminaries. For a $\mathbb{Q}$-algebra $R$, we define the similitude unitary group $\mathrm{GU}(2,2)$ and the similitude orthogonal groups $\mathrm{GO}(4,2)$ by

$$
\begin{aligned}
\mathrm{GU}(2,2)(R) & =\left\{\left.g \in \mathrm{GL}_{4}(K \otimes R)\right|^{t} \bar{g} J g=\lambda(g) J, \lambda(g) \in R^{\times}\right\}, \\
\mathrm{GO}(4,2) & =\left\{\left.g \in \mathrm{GL}_{6}\right|^{t} g Q g=\nu(g) Q, \nu(g) \in \mathrm{GL}_{1}\right\}
\end{aligned}
$$

with

$$
Q=\left(\begin{array}{ccccc} 
& & & 1 & 1 \\
& 2 & 0 & & \\
& & 0 & 2 D & \\
& 1 & & &
\end{array}\right) \in \mathrm{GL}_{6} \quad \text { and } \quad J=\left(\begin{array}{cc}
0 & -\mathbf{1}_{2} \\
\mathbf{1}_{2} & 0
\end{array}\right) \in \mathrm{GL}_{4} .
$$

The homomorphisms $\lambda: \mathrm{GU}(2,2) \rightarrow \mathrm{GL}_{1}$ and $\nu: \mathrm{GO}(4,2) \rightarrow \mathrm{GL}_{1}$ are the similitude norms. We define the subgroups $\operatorname{GSU}(2,2)$ of $\mathrm{GU}(2,2)$ and $\operatorname{GSO}(4,2)$ of $\mathrm{GO}(4,2)$ by

$$
\begin{aligned}
& \operatorname{GSU}(2,2)=\left\{g \in \mathrm{GU}(2,2) \mid \operatorname{det}(g)=\lambda(g)^{2}\right\}, \\
& \operatorname{GSO}(4,2)=\left\{g \in \mathrm{GO}(4,2) \mid \operatorname{det}(g)=\nu(g)^{3}\right\} .
\end{aligned}
$$

For $\alpha \in \operatorname{Res}_{K / \mathbb{Q}}\left(\mathrm{GL}_{1}\right)$, we put

$$
r_{\alpha}=\left(\begin{array}{cccc}
1 & 0 & 0 & 0 \\
0 & \alpha & 0 & 0 \\
0 & 0 & 1 & 0 \\
0 & 0 & 0 & \bar{\alpha}^{-1}
\end{array}\right) \in \mathrm{U}(2,2) .
$$

Then we have an exact sequence

$$
1 \longrightarrow \mathrm{GL}_{1} \stackrel{\iota}{\longrightarrow} \mathrm{GSU}(2,2) \rtimes \operatorname{Res}_{K / \mathbb{Q}}\left(\mathrm{GL}_{1}\right) \stackrel{\rho}{\longrightarrow} \mathrm{GU}(2,2) \longrightarrow 1,
$$


where

$$
\iota(a)=\left(r_{a}, a^{-1}\right) \quad \text { and } \quad \rho(h, \alpha)=h r_{\alpha} .
$$

For each $p$, we put $\mathrm{GU}(2,2)\left(\mathbb{Z}_{\mathfrak{p}}\right)=\mathrm{GU}(2,2)\left(\mathbb{Q}_{p}\right) \cap \mathrm{GL}_{4}\left(\mathfrak{o} \otimes_{\mathbb{Z}} \mathbb{Z}_{p}\right)$ and $\operatorname{GSU}(2,2)\left(\mathbb{Z}_{p}\right)=$ $\mathrm{GU}(2,2)\left(\mathbb{Z}_{p}\right) \cap \mathrm{GSU}(2,2)\left(\mathbb{Q}_{p}\right)$.

Lemma 6.1. For $g \in \mathrm{GU}(2,2)\left(\mathbb{Z}_{p}\right)$, there exist $h \in \mathrm{GSU}(2,2)\left(\mathbb{Z}_{p}\right)$ and $\alpha \in\left(\mathfrak{o} \otimes_{\mathbb{Z}} \mathbb{Z}_{p}\right)^{\times}$such that

$$
g=h r_{\alpha} .
$$

Proof. Let $\mathfrak{o}_{\mathfrak{p}}=\mathfrak{o} \otimes_{\mathbb{Z}} \mathbb{Z}_{p}$. We define the subgroups $H$ and $\mathfrak{o}_{\mathfrak{p}}^{1}$ of $K^{1}\left(\mathbb{Q}_{p}\right)$ by

$$
H=\left\{\operatorname{det}(g) / \lambda(g)^{2} \mid g \in \mathrm{GU}(2,2)\left(\mathbb{Z}_{p}\right)\right\} \quad \text { and } \quad \mathfrak{o}_{\mathfrak{p}}^{1}=\left\{\alpha / \bar{\alpha} \mid \alpha \in \mathfrak{o}_{\mathfrak{p}}^{\times}\right\} .
$$

Note that $H \supset \mathfrak{o}_{\mathfrak{p}}^{1}$. It is enough to show that $H=\mathfrak{o}_{\mathfrak{p}}^{1}$. If $g \in \mathrm{GU}(2,2)\left(\mathbb{Z}_{p}\right)$, then

$$
g^{\prime}=g\left(\begin{array}{cc}
\mathbf{1}_{2} & 0 \\
0 & \lambda(g) \cdot \mathbf{1}_{2}
\end{array}\right)^{-1} \in \mathrm{U}(2,2)\left(\mathbb{Z}_{p}\right)
$$

and we have $\operatorname{det}\left(g^{\prime}\right) / \lambda\left(g^{\prime}\right)^{2}=\operatorname{det}\left(g^{\prime}\right)=\operatorname{det}(g) / \lambda(g)^{2}$. Hence we find that $H=\operatorname{det}\left(\mathrm{U}(2,2)\left(\mathbb{Z}_{p}\right)\right)$.

If $p$ is split in $K / \mathbb{Q}$, we have

$$
K \otimes \mathbb{Q}_{p} \cong \mathbb{Q}_{p} \times \mathbb{Q}_{p}, \quad \mathfrak{o} \otimes_{\mathbb{Z}} \mathbb{Z}_{p} \cong \mathbb{Z}_{p} \times \mathbb{Z}_{p}
$$

and the non-trivial element of $\operatorname{Gal}(K / \mathbb{Q})$ acts by $(x, y) \mapsto(y, x)$. If $g \in \mathrm{U}(2,2)\left(\mathbb{Z}_{p}\right)$, then we have $\operatorname{det}(g)=(x, y)$ for some $x, y \in \mathbb{Z}_{p}^{\times}$. Since $\operatorname{det}(g) \in K^{1}\left(\mathbb{Q}_{p}\right)$, we have $x y=1$, i.e., $y=x^{-1}$. So we have $\operatorname{det}(g)=\alpha / \bar{\alpha}$ with $\alpha=(x, 1) \in \mathfrak{o}_{\mathfrak{p}}^{\times}$. Therefore we have $H=\mathfrak{o}_{\mathfrak{p}}^{1}$.

If $p$ is inert in $K / \mathbb{Q}$, we can regard $p$ as a uniformizer of $K \otimes \mathbb{Q}_{p}$. By Hilbert 90, we have $K^{1}\left(\mathbb{Q}_{p}\right)=\left\{\alpha / \bar{\alpha} \mid \alpha \in K\left(\mathbb{Q}_{p}\right)^{\times}=\left(K \otimes \mathbb{Q}_{p}\right)^{\times}\right\}$. Hence we have $K^{1}\left(\mathbb{Q}_{p}\right)=\mathfrak{o}_{\mathfrak{p}}^{1}$. So we get $H=\mathfrak{o}_{\mathfrak{p}}^{1}$.

Now we assume that $p$ is ramified in $K / \mathbb{Q}$. Let $\mathfrak{p}$ be the prime ideal of $K$ above $p$. Then we have $\mathfrak{o}_{\mathfrak{p}} / \mathfrak{p} \cong \mathbb{F}_{p}$ and the non-trivial element of $\operatorname{Gal}(K / \mathbb{Q})$ acts on $\mathbb{F}_{p}$ by trivially. So we may consider the reduction map

$$
\pi: \mathrm{U}(2,2)\left(\mathbb{Z}_{p}\right) \rightarrow \operatorname{Sp}_{2}\left(\mathbb{F}_{p}\right) .
$$

Let

$$
B=\left\{\left(\begin{array}{cc|cc}
* & * & * & * \\
0 & * & * & * \\
\hline 0 & 0 & * & 0 \\
0 & 0 & * & *
\end{array}\right) \in \mathrm{U}(2,2)\right\}=T N, \quad \mathbf{B}=\left\{\left(\begin{array}{cc|cc}
* & * & * & * \\
0 & * & * & * \\
\hline 0 & 0 & * & 0 \\
0 & 0 & * & *
\end{array}\right) \in \mathrm{Sp}_{2}\right\}
$$

be Borel subgroups of $\mathrm{U}(2,2)$ and $\mathrm{Sp}_{2}$, respectively. We denote by $T$ the torus of diagonal matrices, and by $N$ the unipotent radical of $B$. Let $I=\pi^{-1}\left(\mathbf{B}\left(\mathbb{F}_{p}\right)\right) \subset \mathrm{U}(2,2)\left(\mathbb{Z}_{p}\right)$ denote the Iwahori subgroup of $\mathrm{U}(2,2)\left(\mathbb{Z}_{p}\right)$. Then, the Bruhat decomposition of $\mathrm{Sp}_{2}$ shows that there is a subset $W$ of $\mathrm{U}(2,2)\left(\mathbb{Z}_{p}\right)$ such that $\mathrm{U}(2,2)\left(\mathbb{Z}_{p}\right)$ is generated by $I$ and $W$. We can take $W$ such that $\operatorname{det}(w)=1$ for all $w \in W$. Hence we have $H=\operatorname{det}\left(\mathrm{U}(2,2)\left(\mathbb{Z}_{p}\right)\right)=\operatorname{det}(I)$. Moreover, by the Iwahori decomposition, we have

$$
I=\left(I \cap N^{-}\right)(I \cap T)(I \cap N),
$$

where $N^{-}$is the opposite of $N$. Since $\operatorname{det}(N)=\operatorname{det}\left(N^{-}\right)=\{1\}$, we have $\operatorname{det}(I)=\operatorname{det}(I \cap T)$. Clearly, this is equal to $\mathfrak{o}_{\mathfrak{p}}^{1}$. Therefore we have $H=\mathfrak{o}_{p}^{1}$.

As in $[21] \S 2.1$, we set the six dimensional vector space over $\mathbb{Q}$ by

$$
V=\left\{B\left(x_{1}, \ldots, x_{6}\right) \in \mathrm{M}_{4}(K) \mid x_{i} \in \mathbb{Q}(1 \leq i \leq 6)\right\}
$$

where $B\left(x_{1}, \ldots, x_{6}\right)$ is defined by

$$
B\left(x_{1}, \ldots, x_{6}\right)=\left(\begin{array}{cccc}
0 & x_{1} & x_{3}+x_{4} \sqrt{-D} & x_{2} \\
-x_{1} & 0 & x_{5} & -x_{3}+x_{4} \sqrt{-D} \\
-x_{3}-x_{4} \sqrt{-D} & -x_{5} & 0 & x_{6} \\
-x_{2} & x_{3}-x_{4} \sqrt{-D} & -x_{6} & 0
\end{array}\right) .
$$


We define a mapping $\Psi: V \rightarrow \mathbb{Q}$ by $\Psi(B)=\operatorname{Tr}\left(B J^{t} \bar{B} J\right)$. As in [21] $\S 2.1$, we have

$$
\Psi\left(B\left(x_{1}, \ldots, x_{6}\right)\right)=-4 Q\left[{ }^{t}\left(x_{1}, \ldots, x_{6}\right)\right] .
$$

As a basis for $V$, we may take $e_{i}=B\left(\delta_{i, 1}, \ldots, \delta_{i, 6}\right)$ for $1 \leq i \leq 6$. Then with respect to the basis $\left\{e_{i} \mid 1 \leq i \leq 6\right\}$, we may identify $\mathrm{GO}(V)$ as $\mathrm{GO}(4,2)$.

We define homomorphisms

$$
\begin{array}{rlrl}
\phi_{1}: \operatorname{GSU}(2,2) & \rightarrow \operatorname{GSO}(V) \cong \operatorname{GSO}(4,2), & \phi_{1}(g): V \ni B \rightarrow g B^{t} g \in V, \\
\phi_{2}: K^{\times} \rightarrow \operatorname{GSO}(V) \cong \operatorname{GSO}(4,2), & \phi_{2}(\alpha): V \ni B \rightarrow \bar{\alpha} r_{\alpha} B r_{\alpha} \in V .
\end{array}
$$

Note that $\nu\left(\phi_{1}(g)\right)=\lambda(g)^{2}$ for $g \in \operatorname{GSU}(2,2)$ and $\nu\left(\phi_{2}(\alpha)\right)=N_{K / \mathbb{Q}}(\alpha)$ for $\alpha \in K^{\times}$. For $\alpha=x+y \sqrt{-D} \in K^{\times}$, we have

$$
\phi_{2}(\alpha)=\left(\begin{array}{cccccc}
N_{K / \mathbb{Q}}(\alpha) & & & & & \\
& 1 & & & & \\
& & x & D y & & \\
& -y & x & & \\
& & & & N_{K / \mathbb{Q}}(\alpha) & \\
& & & & & 1
\end{array}\right) \in \operatorname{GSO}(4,2) .
$$

We find that $\phi_{1}\left(\operatorname{GSU}(2,2)\left(\mathbb{Z}_{p}\right)\right) \subset \mathrm{GSO}(V)\left(\mathbb{Z}_{p}\right)$. However, in general, it is not true that

$$
\alpha \in \mathfrak{o} \otimes \mathbb{Z}_{p} \Rightarrow \phi_{2}(\alpha) \in \operatorname{GSO}(V)\left(\mathbb{Z}_{p}\right) .
$$

For $g \in \mathrm{GU}(2,2)$, we decompose $g=h r_{\alpha}$ with $h \in \mathrm{GSU}(2,2)$ and $\alpha \in K^{\times}$. Then the image of $\phi_{1}(h) \phi_{2}(\alpha)$ to PGSO $(4,2)$ does not depend on the choice of $(h, \alpha)$. We denote this element by $\phi(g) \in \operatorname{PGSO}(4,2)$. Note that for all $\alpha \in K^{\times}$, we have $\phi\left(\alpha \mathbf{1}_{4}\right)=1$ in $\operatorname{PGSO}(4,2)$ and $\phi$ induces an isomorphism

$$
\phi: \operatorname{PGU}(2,2) \stackrel{\cong}{\rightarrow} \operatorname{PGSO}(4,2) .
$$

6.2. Theta lifts. Let $\mathcal{K}=\mathrm{GU}(2,2)\left(\mathbb{A}_{\mathbb{Q}, \text { fin }}\right) \cap\left(\prod_{p} \mathrm{GL}_{4}\left(\mathfrak{o}_{p}\right)\right)$, where $\mathfrak{o}_{p}=\mathfrak{o} \otimes \mathbb{Z}_{p}$. Put $\mathcal{K}_{0}=$ $\mathcal{K} \cap \mathrm{U}(2,2)\left(\mathbb{A}_{\mathbb{Q}}\right)$. We easily find that the canonical injection $\mathrm{U}(2,2)\left(\mathbb{A}_{\mathbb{Q}}\right) \hookrightarrow \mathrm{GU}(2,2)\left(\mathbb{A}_{\mathbb{Q}}\right)$ induces a bijection

$$
\mathrm{U}(2,2)(\mathbb{Q}) \backslash \mathrm{U}(2,2)\left(\mathbb{A}_{\mathbb{Q}}\right) / \mathrm{U}(2,2)(\mathbb{R}) \mathcal{K}_{0} \rightarrow \mathrm{GU}(2,2)(\mathbb{Q}) \backslash \mathrm{GU}(2,2)\left(\mathbb{A}_{\mathbb{Q}}\right) / \mathrm{GU}(2,2)(\mathbb{R})^{+} \mathcal{K}
$$

By the Chebotarev density theorem, as a complete system of representatives of $C l_{K}$, we can take integral ideals $\mathfrak{c}_{1}=\mathfrak{o}, \mathfrak{c}_{2}, \ldots, \mathfrak{c}_{h}$ which are prime to $2 D$. We choose $t_{i}=\left(t_{i, \mathfrak{p}}\right)_{\mathfrak{p}} \in \mathbb{A}_{K, \text { fin }}^{\times}$ such that $\operatorname{ord}_{\mathfrak{p}}\left(t_{i, \mathfrak{p}}\right)=\operatorname{ord}_{\mathfrak{p}}\left(\mathfrak{c}_{i}\right)$ for all prime ideals $\mathfrak{p}$ of $K$. We put $\gamma_{i}=r_{t_{i}} \in \mathrm{U}(2,2)\left(\mathbb{A}_{\mathbb{Q}}\right)$ and $C_{i}=N\left(\mathfrak{c}_{i}\right) \in \mathbb{Z}_{>0}$. Then by Lemma 13.1 of [17], the set $\left\{\gamma_{1}, \ldots, \gamma_{h}\right\}$ gives a complete system of $\mathrm{U}(2,2)(\mathbb{Q}) \backslash \mathrm{U}(2,2)\left(\mathbb{A}_{\mathbb{Q}}\right) / \mathrm{U}(2,2)(\mathbb{R}) \mathcal{K}_{0}$.

Let $f \in S_{2 \kappa+1}\left(\Gamma_{0}(D), \chi\right)$ be a normalized Hecke eigenform and $F_{\mathfrak{c}_{i}}$ the hermitian Maass lift of $f$ defined in Sect. 2. Then by a result of Ikeda ([17] Theorem 13.2 and Theorem 15.18), there is an automorphic form Lift $^{(2)}(f)$ on $\mathrm{U}(2,2)\left(\mathbb{A}_{\mathbb{Q}}\right)$ defined by

$$
\operatorname{Lift}^{(2)}(f)\left(u \gamma_{i} x k\right)=C_{i}^{-\kappa-1}\left(F_{\mathfrak{c}_{i}} \|_{2 \kappa+2} x\right)(\mathbf{i})=C_{i}^{-\kappa-1} F_{\mathfrak{c}_{i}}(x\langle\mathbf{i}\rangle) j(x, \mathbf{i})^{-2 \kappa-2}(\operatorname{det} x)^{\kappa+1}
$$

for $u \in \mathrm{U}(2,2)(\mathbb{Q}), x \in \mathrm{U}(2,2)(\mathbb{R})$ and $k \in \mathcal{K}_{0}$. Here, we put $\mathbf{i}=\sqrt{-1} \cdot \mathbf{1}_{4} \in \mathcal{H}_{2}$.

Let $\mathbf{f}$ be the cusp form on $\mathrm{GL}_{2}\left(\mathbb{A}_{\mathbb{Q}}\right)$ given by $f$. We define $\varphi=\otimes_{v} \varphi_{v} \in \mathcal{S}\left(V\left(\mathbb{A}_{\mathbb{Q}}\right)\right)$ as follows:

- If $v=p<\infty$ and $p \neq 2$, then $\varphi_{p}$ is the characteristic function of $V\left(\mathbb{Z}_{p}\right)$.

- If $v=2$, then $\varphi_{2}$ is the characteristic function of

$$
L= \begin{cases}V\left(\mathbb{Z}_{2}\right) \cup\left(\mathbb{Z}_{2} e_{1}+\mathbb{Z}_{2} e_{2}+2^{-1} \mathbb{Z}_{2}^{\times} e_{3}+2^{-1} \mathbb{Z}_{2}^{\times} e_{4}+\mathbb{Z}_{2} e_{5}+\mathbb{Z}_{2} e_{6}\right) & \text { if } D \text { is odd }, \\ V\left(\mathbb{Z}_{2}\right)+2^{-1} \mathbb{Z}_{2} e_{4} & \text { if } D \text { is even. }\end{cases}
$$

- If $v=\infty$, then

$$
\varphi_{\infty}\left(x_{1}, \ldots, x_{6}\right)=\left(-\sqrt{-1} x_{1}+x_{2}-x_{5}+\sqrt{-1} x_{6}\right)^{2 \kappa+2} e^{-\pi\left(x_{1}^{2}+x_{2}^{2}+2 x_{3}^{2}+2 D x_{4}^{2}+x_{5}^{2}+x_{6}^{2}\right)} .
$$


We consider the theta lift $\theta(\mathbf{f}, \varphi)$ on $\operatorname{GO}(V)\left(\mathbb{A}_{\mathbb{Q}}\right)$. Recall that $\theta(\mathbf{f}, \varphi)$ is defined by

$$
\theta(h ; \mathbf{f}, \varphi)=\int_{\left[\mathrm{SL}_{2}\right]} \theta\left(\alpha \cdot \alpha_{h}, h ; \varphi\right) \mathbf{f}\left(\alpha \cdot \alpha_{h}\right) d \alpha
$$

for $h \in \mathrm{GO}(V)\left(\mathbb{A}_{\mathbb{Q}}\right)$, where $\alpha_{h}$ is an element in $\mathrm{GL}_{2}\left(\mathbb{A}_{\mathbb{Q}}\right)$ satisfying $\operatorname{det}\left(\alpha_{h}\right)=\nu(h)$. In particular, we can take $\alpha_{h}=d(\nu(h))$. Note that $\nu\left(\operatorname{GSO}(V)\left(\mathbb{A}_{\mathbb{Q}}\right)\right)=N_{K / \mathbb{Q}}\left(\mathbb{A}_{K}^{\times}\right)$. Since the central character of $\mathbf{f}$ is $\underline{\chi}=\chi_{V}$, by [11] Lemma 5.1.9, the center of $\operatorname{GSO}(4,2)$ acts on $\theta(\mathbf{f}, \varphi)$ trivially, i.e., the quantity

$$
\theta(\phi(g) ; \mathbf{f}, \varphi)=\int_{\left[\mathrm{SL}_{2}\right]} \theta\left(\alpha \cdot d\left(\nu\left(h_{g}\right)\right), h_{g} ; \varphi\right) \mathbf{f}\left(\alpha \cdot d\left(\nu\left(h_{g}\right)\right)\right) d \alpha
$$

does not depend on the choice of a representative $h_{g} \in \operatorname{GSO}(V)\left(\mathbb{A}_{\mathbb{Q}}\right)$ of $\phi(g) \in \operatorname{PGSO}(V)\left(\mathbb{A}_{\mathbb{Q}}\right)$. Hence we may consider the function $\theta(\phi(g) ; \mathbf{f}, \varphi)$ on $\mathrm{GU}(2,2)\left(\mathbb{A}_{\mathbb{Q}}\right)$.

Proposition 6.2. For all $g \in \mathrm{U}(2,2)\left(\mathbb{A}_{\mathbb{Q}}\right)$, we have

$$
\theta(\phi(g) ; \mathbf{f}, \varphi)=\left(\prod_{p \mid D} q_{p}^{-1}\right) \cdot 2^{2 \kappa+2} a_{f}(D)^{-1} \operatorname{Lift}^{(2)}(f)(g),
$$

where $q_{p}=\left(\mathrm{SL}_{2}\left(\mathbb{Z}_{p}\right): K_{0}\left(D ; \mathbb{Z}_{p}\right)\right)$.

The rest of this section is devoted to the proof of Proposition 6.2. The proof is similar to that of Lemma 7.1 of [14]. To prove this proposition, we compare Fourier coefficients of $\theta(\phi(g) ; \mathbf{f}, \varphi)$ with those of Lift $^{(2)}(f)$.

6.3. Fourier coefficients of Lift $^{(2)}(f)$. For $B \in \operatorname{Her}_{2}(\mathbb{Q})$, we define the $B$-th Fourier coefficient $\mathcal{W}_{\mathbf{F}, B}\left(\operatorname{resp} . \mathcal{W}_{B}\right)$ of $\mathbf{F}=$ Lift $^{(2)}(f)(\operatorname{resp} . \theta(\mathbf{f}, \varphi))$ by

$$
\mathcal{W}_{\mathbf{F}, B}(g)=\int_{\left[\mathrm{Her}_{2}\right]} \mathbf{F}(n(X) g) \overline{\psi(\operatorname{Tr}(B X))} d X
$$

for $g \in \mathrm{U}(2,2)\left(\mathbb{A}_{\mathbb{Q}}\right)($ resp.

$$
\mathcal{W}_{B}(g)=\int_{\left[\mathrm{Her}_{2}\right]} \theta(\phi(n(X) g) ; \mathbf{f}, \varphi) \overline{\psi(\operatorname{Tr}(B X))} d X
$$

for $\left.g \in \mathrm{GU}(2,2)\left(\mathbb{A}_{\mathbb{Q}}\right)\right)$. Here we put

$$
n(X)=\left(\begin{array}{cc}
\mathbf{1}_{2} & X \\
0 & \mathbf{1}_{2}
\end{array}\right) \in \mathrm{U}(2,2) \quad \text { for } X \in \mathrm{Her}_{2} .
$$

Then, we have

$$
\mathbf{F}(g)=\sum_{B \in \operatorname{Her}_{2}(\mathbb{Q})} \mathcal{W}_{\mathbf{F}, B}(g) \text { and } \theta(\phi(g) ; \mathbf{f}, \varphi)=\sum_{B \in \operatorname{Her}_{2}(\mathbb{Q})} \mathcal{W}_{B}(g) .
$$

First, we compute $\mathcal{W}_{\mathbf{F}, B}$ in Proposition 6.5. We define $\mathbb{K}=\{g \in \mathrm{U}(2,2)(\mathbb{R}) \mid g\langle\mathbf{i}\rangle=\mathbf{i}\}$ and $\mathbb{K}_{0}=\mathbb{K} \cap \mathrm{SU}(2,2)(\mathbb{R})$.

Lemma 6.3. We have

$$
\mathbb{K}=\left\{\left(\begin{array}{cc}
\alpha & \beta \\
-\beta & \alpha
\end{array}\right) \mid \alpha, \beta \in \mathrm{M}_{2}(\mathbb{C}),{ }^{t} \bar{\alpha} \beta={ }^{t} \bar{\beta} \alpha,{ }^{t} \bar{\alpha} \alpha+{ }^{t} \bar{\beta} \beta=1\right\} .
$$

Proof. Let $k=\left(\begin{array}{ll}A & B \\ C & D\end{array}\right) \in \mathbb{K}$ with $A, B, C, D \in \mathrm{M}_{2}(\mathbb{C})$. We put

$$
\begin{aligned}
& X=A \sqrt{-1}+B=-C+D \sqrt{-1} \\
& Y=A \sqrt{-1}+C=-B+D \sqrt{-1} \\
& Z=B+C=(D-A) \sqrt{-1}
\end{aligned}
$$

Then, it is easily to check that ${ }^{t} \bar{X} X=\mathbf{1}_{2},{ }^{t} \bar{Y} Y=\mathbf{1}_{2}$ and ${ }^{t} \bar{Z} Z={ }^{t} \bar{Y} Y-\mathbf{1}_{2}=\mathbf{0}_{2}$. Hence $Z=\mathbf{0}_{2}$. 
For $X \in \operatorname{Her}_{2}(\mathbb{R})$ and $A \in \mathrm{GL}_{2}(\mathbb{C})$, we put

$$
n(X)=\left(\begin{array}{ll}
\mathbf{1}_{2} & X \\
& \mathbf{1}_{2}
\end{array}\right) \text { and } m(A)=\left(\begin{array}{ll}
A & \\
& t^{A}
\end{array}{ }^{-1}\right) .
$$

Lemma 6.4. Any element $g$ in $\mathrm{GU}(2,2)(\mathbb{R})^{+}$can be written as

$$
g=z \mathbf{1}_{4} \cdot n(X) \cdot m(A) \cdot k \cdot r_{t},
$$

where $z \in \mathbb{C}^{\times}, X \in \operatorname{Her}_{2}(\mathbb{R}), A \in \mathrm{GL}_{2}(\mathbb{C})$ with $\operatorname{det}(A) \in \mathbb{R}^{\times}, k \in \mathbb{K}_{0}$ and $t \in \mathbb{C}^{\times}$with $|t|=1$.

Proof. Since $\mathrm{GU}(2,2)(\mathbb{R})^{+}$is generated by its center and $\mathrm{U}(2,2)(\mathbb{R})$, it suffices to consider $g \in \mathrm{U}(2,2)(\mathbb{R})$. Since $Z=g\langle\mathbf{i}\rangle \in \mathcal{H}_{2}$, we fined that $\left(Z+{ }^{t} \bar{Z}\right) / 2$ is a hermitian matrix and $\left(Z-{ }^{t} \bar{Z}\right) /(2 \sqrt{-1})$ is a positive definite hermitian matrix. So there exist $A \in \mathrm{GL}_{2}(\mathbb{C})$ and $X \in \mathrm{Her}_{2}(\mathbb{R})$ such that

$$
g\langle\mathbf{i}\rangle=A^{t} \bar{A} \mathbf{i}+X=(n(X) \cdot m(A))\langle\mathbf{i}\rangle .
$$

Hence we find $k=(n(X) \cdot m(A))^{-1} g \in \mathbb{K}$. Since $\operatorname{det}(m(A))=\operatorname{det}(A) \overline{\operatorname{det}(A)}^{-1}$, there exists $\theta \in \mathbb{R}$ such that $e^{4 \sqrt{-1} \theta}=\operatorname{det}(m(A))$. Then, we find that $\operatorname{det}\left(e^{-\sqrt{-1} \theta} A\right) \in \mathbb{R}$ and $e^{\sqrt{-1} \theta} \mathbf{1}_{4} \in \mathbb{K}$. Therefore we have the decomposition

$$
g=n(X) \cdot m\left(e^{-\sqrt{-1} \theta} A\right) \cdot\left(e^{\sqrt{-1} \theta} k\right) .
$$

We put $k^{\prime}=e^{\sqrt{-1} \theta} k \in \mathbb{K}$. Since $N_{\mathbb{C} / \mathbb{R}}\left(\operatorname{det}\left(k^{\prime}\right)\right)=1$, there exists $t \in \mathbb{C}^{\times}$so that $|t|=1$ and $\operatorname{det}\left(k^{\prime}\right)=t^{2}=\operatorname{det}\left(r_{t}\right)$. Hence we have $k^{\prime}\left(r_{t}\right)^{-1} \in \mathbb{K}_{0}$.

The action of $r_{t}$ on $\mathbf{F}$ is trivial for all $t \in \mathbb{C}^{\times}$with $|t|=1$. Let

$$
k=\left(\begin{array}{cc}
\alpha & \beta \\
-\beta & \alpha
\end{array}\right) \in \mathbb{K}_{0} .
$$

Since $\operatorname{det}(k)=\operatorname{det}(\alpha+\sqrt{-1} \beta)(\alpha-\sqrt{-1} \beta)=1$, the action of $k$ on $\mathbf{F}$ is the scalar multiplication of

$$
j(k, \mathbf{i})^{-2 \kappa-2} \operatorname{det}(k)^{\kappa+1}=\operatorname{det}(\alpha-\sqrt{-1} \beta)^{-2 \kappa-2}=\operatorname{det}(\alpha+\sqrt{-1} \beta)^{2 \kappa+2} .
$$

Proposition 6.5. Let $x_{\infty}=n(X) \cdot m(A) \in \mathrm{U}(2,2)(\mathbb{R})$, where $X \in \operatorname{Her}_{2}(\mathbb{R})$ and $A \in \mathrm{GL}_{2}(\mathbb{C})$ with $\operatorname{det}(A) \in \mathbb{R}^{\times}$. Put $Y=A^{t} \bar{A}$. Then,

$$
\mathcal{W}_{\mathbf{F}, B}\left(\gamma_{i} x_{\infty}\right)=C_{i}^{-\kappa-1} \operatorname{det}(Y)^{\kappa+1} A_{F_{\mathrm{c}_{i}}}(B) \exp (2 \pi \sqrt{-1} \operatorname{Tr}(B(X+Y \sqrt{-1}))) .
$$

Here, we set $A_{F_{\mathfrak{c}_{i}}}(B)=0$ if $B \notin \Lambda_{2}^{\mathfrak{c}_{i}}(\mathfrak{o})^{+}$.

Proof. We fix a $\mathbb{Z}$-basis $\left\{\lambda_{1}, \lambda_{2}\right\}$ of $\overline{\mathfrak{c}}_{i}$, i.e., $\overline{\mathfrak{c}}_{i}=\lambda_{1} \mathbb{Z}+\lambda_{2} \mathbb{Z}$. As a coordinate of $\mathrm{Her}_{2}$, we use

$$
Z=\left(\begin{array}{cc}
\frac{n}{\lambda_{1} a+\lambda_{2} b} & \lambda_{1} a+\lambda_{2} b \\
C_{i} m
\end{array}\right) \in \operatorname{Her}_{2} .
$$

Then,

$$
\begin{aligned}
\mathcal{W}_{\mathbf{F}, B}\left(\gamma_{i} x_{\infty}\right) & =\int_{\left[\mathrm{Her}_{2}\right]} \mathbf{F}\left(n(Z) \gamma_{i} x_{\infty}\right) \overline{\psi(\operatorname{Tr}(B Z))} d Z \\
& =\int_{(\hat{\mathbb{Z}} \times[0,1))^{4}} \mathbf{F}\left(\gamma_{i} n\left(d\left(t_{i}\right)^{-1} Z d\left(\overline{t_{i}}\right)^{-1}\right) x_{\infty}\right) \overline{\psi(\operatorname{Tr}(B Z))} d Z .
\end{aligned}
$$

Write $Z=Z_{\text {fin }}+Z_{\infty}$ with $Z_{\text {fin }} \in \operatorname{Her}_{2}\left(\mathbb{A}_{\mathbb{Q}, \text { fin }}\right)$ and $Z_{\infty} \in \operatorname{Her}_{2}(\mathbb{R})$. If $a, b, n, m \in \hat{\mathbb{Z}}$, then we find that $n\left(d\left(t_{i}\right)^{-1} Z_{\text {fin }} d\left(\bar{t}_{i}\right)^{-1}\right) \in \mathcal{K}_{0}$. Hence $\mathcal{W}_{\mathbf{F}, B}\left(\gamma_{i} x_{\infty}\right)$ is equal to

$$
\left(\int_{\hat{\mathbb{Z}}^{4}} \overline{\psi\left(\operatorname{Tr}\left(B Z_{\mathrm{fin}}\right)\right)} d Z_{\mathrm{fin}}\right)\left(\int_{[0,1)^{4}} \mathbf{F}\left(\gamma_{i} n\left(Z_{\infty}\right) x_{\infty}\right) \overline{\psi\left(\operatorname{Tr}\left(B Z_{\infty}\right)\right)} d Z_{\infty}\right) .
$$

The first integral is not vanish if and only if $\operatorname{Tr}\left(B Z_{\text {fin }}\right) \in \hat{\mathbb{Z}}$ whenever $a, b, n, m \in \hat{\mathbb{Z}}$. This condition is equivalent to the condition that $B \in \Lambda_{2}^{\mathfrak{c}_{i}}(\mathfrak{o})$. In this case, the first integral is equal to 1 . 
Since $\mathbf{F}\left(\gamma_{i} n\left(Z_{\infty}\right) x_{\infty}\right)=C_{i}^{-\kappa-1} \operatorname{det}(Y)^{\kappa+1} F_{\mathfrak{c}_{i}}\left(X+Y \sqrt{-1}+Z_{\infty}\right)$, the second integral is equal to

$$
\begin{aligned}
C_{i}^{-\kappa-1} \operatorname{det}(Y)^{\kappa+1} \sum_{H \in \Lambda_{2}^{\mathfrak{c}_{i}}(\mathfrak{o})^{+}} A_{F_{\mathfrak{c}_{i}}}(H) \exp (2 \pi \sqrt{-1} \operatorname{Tr}(H(X+Y \sqrt{-1}))) \\
\times \int_{[0,1)^{4}} \exp \left(2 \pi \sqrt{-1} \operatorname{Tr}\left((H-B) Z_{\infty}\right)\right) d Z_{\infty} .
\end{aligned}
$$

Since $H-B \in \Lambda_{2}^{\mathfrak{c}_{i}}(\mathfrak{o})$, we find that

$$
\int_{[0,1)^{4}} \exp \left(2 \pi \sqrt{-1} \operatorname{Tr}\left((H-B) Z_{\infty}\right)\right) d Z_{\infty}= \begin{cases}1 & \text { if } B=H, \\ 0 & \text { if } B \neq H .\end{cases}
$$

This completes the proof.

6.4. Fourier coefficients of $\theta(\mathbf{f}, \varphi)$. Next we compute $\mathcal{W}_{B}(g)$. Let $V_{0}=\left\langle e_{1}, e_{6}\right\rangle$ and $V_{1}=$ $\left\langle e_{2}, e_{3}, e_{4}, e_{5}\right\rangle$ be subspaces of $V$. We identify $V_{0}$ (resp. $V_{1}$ ) with the space of column vectors $\mathbb{Q}^{2}$ (resp. $\left.\mathbb{Q}^{4}\right)$ via this basis. For

$$
n(X)=\left(\begin{array}{cc}
\mathbf{1}_{2} & X \\
0 & \mathbf{1}_{2}
\end{array}\right) \in \mathrm{SU}(2,2) \quad \text { with } \quad X=\left(\begin{array}{cc}
x_{1} & x_{2}+\sqrt{-D} x_{3} \\
x_{2}-\sqrt{-D} x_{3} & x_{4}
\end{array}\right) \in \mathrm{Her}_{2},
$$

we have

$$
\phi_{1}(n(X))=\left(\begin{array}{cccccc}
1 & x_{4} & 2 x_{2} & 2 D x_{3} & -x_{1} & x_{1} x_{4}-x_{2}^{2}-D x_{3}^{2} \\
0 & 1 & 0 & 0 & 0 & x_{1} \\
0 & 0 & 1 & 0 & 0 & -x_{2} \\
0 & 0 & 0 & 1 & 0 & -x_{3} \\
0 & 0 & 0 & 0 & 1 & -x_{4} \\
0 & 0 & 0 & 0 & 0 & 1
\end{array}\right) .
$$

There are two isomorphisms of the $\mathbb{Q}$-vector spaces

$$
\begin{aligned}
& v: \operatorname{Her}_{2}(\mathbb{Q}) \rightarrow V_{1}(\mathbb{Q}) \cong \mathbb{Q}^{4}, X=\left(\begin{array}{cc}
x_{1} & x_{2}+\sqrt{-D} x_{3} \\
x_{2}-\sqrt{-D} x_{3} & x_{4}
\end{array}\right) \mapsto\left(\begin{array}{c}
x_{1} \\
-x_{2} \\
-x_{3} \\
-x_{4}
\end{array}\right), \\
& \beta: \operatorname{Her}_{2}(\mathbb{Q}) \rightarrow V_{1}(\mathbb{Q}) \cong \mathbb{Q}^{4}, B=\left(\begin{array}{cc}
b_{1} & b_{2}+\sqrt{-D} b_{3} \\
b_{2}-\sqrt{-D} b_{3} & b_{4}
\end{array}\right) \mapsto\left(\begin{array}{c}
-b_{4} \\
-b_{2} \\
-b_{3} \\
b_{1}
\end{array}\right) .
\end{aligned}
$$

Put $v=v(X)$ and $\beta=\beta(B)$. Then we have

$$
\phi_{1}(n(X))=\ell(v), \quad \operatorname{Tr}(B X)=(\beta, v), \quad-Q_{1}[\beta]=\operatorname{det}(B) .
$$

Hence we have $\mathcal{W}_{B}(g)=\mathcal{W}_{\beta}\left(h_{g}\right)$, where $\mathcal{W}_{\beta}$ is the $\beta$-th Fourier coefficient of $\theta(\mathbf{f}, \varphi)$ defined in Sect. 5.4.

For $B=0$, by Paul's result [22], we get the following lemma.

Lemma 6.6. For all $g \in \mathrm{GU}(2,2)\left(\mathbb{A}_{\mathbb{Q}}\right)$, we have

$$
\mathcal{W}_{0}(g)=0 .
$$

Proof. Let $\pi_{f} \cong \otimes_{v}^{\prime} \pi_{f, v}$ be the automorphic representation of $\mathrm{GL}_{2}\left(\mathbb{A}_{\mathbb{Q}}\right)$ generated by $\mathbf{f}$. We write $\mathbf{f}=\otimes_{v}^{\prime} \mathbf{f}_{v}$ with $\mathbf{f}_{v} \in \pi_{f, v}$. As we noted in Sect. 4 , we have $\left.\pi_{f, \infty}\right|_{\mathrm{SL}_{2}(\mathbb{R})} \cong \pi_{f}^{+} \oplus \pi_{f}^{-}$and $\mathbf{f}_{\infty} \in \pi_{f}^{+}$, where $\pi_{f}^{+}$(resp. $\pi_{f}^{-}$) is the holomorphic (resp. anti-holomorphic) discrete series of $\mathrm{SL}_{2}(\mathbb{R})$ with minimal weight $2 \kappa+1$ (resp. $-(2 \kappa+1)$ ). Let $g \in \mathrm{GU}(2,2)\left(\mathbb{A}_{\mathbb{Q}}\right)$ and take a representative $h_{g} \in \operatorname{GSO}(V)\left(\mathbb{A}_{\mathbb{Q}}\right)$ of $\phi(g) \in \operatorname{PGSO}(V)\left(\mathbb{A}_{\mathbb{Q}}\right)$. Fix $\varphi_{0} \in \mathcal{S}\left(V_{0}\left(\mathbb{A}_{\mathbb{Q}}\right)\right)$ and $\varphi_{1}^{\prime} \in \otimes_{p<\infty}^{\prime} \mathcal{S}\left(V_{1}\left(\mathbb{Q}_{p}\right)\right)$. Consider the linear map

$$
\Phi: \omega_{V_{1}(\mathbb{R}), \psi_{\infty}} \otimes \pi_{f}^{+} \rightarrow \mathbb{C}, \varphi_{1} \otimes f_{\infty} \mapsto \mathcal{W}_{0}\left(g ; \theta\left(f_{\infty} \otimes\left(\otimes_{p<\infty}^{\prime} \mathbf{f}_{p}\right), \varphi_{0} \otimes\left(\varphi_{1} \otimes \varphi_{1}^{\prime}\right)\right)\right),
$$


where $\mathcal{W}_{0}\left(g ; \theta\left(\mathbf{f}^{\prime}, \varphi^{\prime}\right)\right)=\mathcal{W}_{0}\left(h_{g} ; \theta\left(\mathbf{f}^{\prime}, \varphi^{\prime}\right)\right)$ is the 0 -th Fourier coefficient of $\theta\left(\mathbf{f}^{\prime}, \varphi^{\prime}\right)$ for $\mathbf{f}^{\prime} \in \pi$ and $\varphi^{\prime} \in \mathcal{S}\left(V\left(\mathbb{A}_{\mathbb{Q}}\right)\right)$. Note that this integral does not depend on the choice of $h_{g}$ since $\mathbf{f}^{\prime}=$ $f_{\infty} \otimes\left(\otimes_{p<\infty}^{\prime} \mathbf{f}_{p}\right)$ has the central character $\underline{\chi}$. It suffices to show that $\Phi=0$.

Now, we claim that the linear map $\Phi: \omega_{V_{1}(\mathbb{R}), \psi_{\infty}} \otimes \pi_{f}^{+} \rightarrow \mathbb{C}$ is $\mathrm{SL}_{2}\left(\mathbb{A}_{\mathbb{Q}}\right)$-invariant. Indeed, by Lemma $5.1(2)$, we find that $\mathcal{W}_{0}\left(g ; \theta\left(\mathbf{f}^{\prime}, \varphi^{\prime}\right)\right)$ is equal to

$$
\int_{\left[\mathrm{SL}_{2}\right]} \sum_{x_{1} \in V_{1}(\mathbb{Q})} \hat{\omega}\left(\alpha \cdot d\left(\nu\left(h_{g}\right)\right), h_{g}\right)\left(\varphi^{\prime}\right)^{\wedge}\left(x_{1} ; 0,0\right) \mathbf{f}^{\prime}\left(\alpha \cdot d\left(\nu\left(h_{g}\right)\right)\right) d \alpha .
$$

We put

$$
\hat{\varphi}_{0}\left(y_{1}, y_{2}\right)=\int_{\mathbb{A} Q} \varphi_{0}\left(z, y_{1}\right) \psi\left(y_{2} z\right) d z \text {. }
$$

Then we have $\left(\varphi_{0} \otimes\left(\varphi_{1} \otimes \varphi_{1}^{\prime}\right)\right)^{\wedge}\left(x_{1} ; y_{1}, y_{2}\right)=\hat{\varphi}_{0}\left(y_{1}, y_{2}\right) \cdot\left(\varphi_{1} \otimes \varphi_{1}^{\prime}\right)\left(x_{1}\right)$. As we noted in Sect. 5.4, we then find that

$$
\hat{\omega}\left(\alpha_{0}, 1\right)\left(\varphi_{0} \otimes\left(\varphi_{1} \otimes \varphi_{1}^{\prime}\right)\right)^{\wedge}\left(x_{1} ; y\right)=\hat{\varphi}_{0}\left(y \alpha_{0}\right) \cdot\left[\omega_{V_{1}\left(\mathbb{A}_{\mathbb{Q}}\right), \psi}\left(\alpha_{0}, 1\right)\left(\varphi_{1} \otimes \varphi_{1}^{\prime}\right)\right]\left(x_{1}\right)
$$

for $y \in V_{0}\left(\mathbb{A}_{\mathbb{Q}}\right) \cong \mathbb{A}_{\mathbb{Q}}^{2}$ and $\alpha_{0} \in \mathrm{SL}_{2}\left(\mathbb{A}_{\mathbb{Q}}\right)$. In particular, for $\alpha_{0} \in \mathrm{SL}_{2}(\mathbb{R})$, we have

$$
\begin{aligned}
\hat{\omega}\left(\alpha_{0}, 1\right)\left(\varphi_{0} \otimes\left(\varphi_{1} \otimes \varphi_{1}^{\prime}\right)\right)^{\wedge}\left(x_{1} ; 0,0\right) & =\hat{\varphi}_{0}(0,0) \cdot\left[\omega_{V_{1}\left(\mathbb{A}_{\mathbb{Q}}\right), \psi}\left(\alpha_{0}, 1\right)\left(\varphi_{1} \otimes \varphi_{1}^{\prime}\right)\right]\left(x_{1}\right) \\
& =\left(\varphi_{0} \otimes\left(\left[\omega_{V_{1}(\mathbb{R}), \psi_{\infty}}\left(\alpha_{0}, 1\right) \varphi_{1}\right] \otimes \varphi_{1}^{\prime}\right)\right)^{\wedge}\left(x_{1} ; 0,0\right) .
\end{aligned}
$$

This implies that

$$
\Phi\left(\left[\omega_{V_{1}(\mathbb{R}), \psi_{\infty}}\left(\alpha_{0}, 1\right) \varphi_{1}\right] \otimes\left[\pi_{f}^{+}\left(\alpha_{0}\right) f_{\infty}\right]\right)=\Phi\left(\varphi_{1} \otimes f_{\infty}\right)
$$

for all $\alpha_{0} \in \mathrm{SL}_{2}(\mathbb{R})$ as desired.

We put $\mathfrak{g}=\operatorname{Lie}\left(\mathrm{SL}_{2}(\mathbb{R})\right) \otimes_{\mathbb{R}} \mathbb{C}$ and $K=\mathrm{SO}(2)$. By [22] Theorem 15 and Corollary 23, we find that

$$
\operatorname{Hom}_{(\mathfrak{g}, K)}\left(\omega_{V_{1}(\mathbb{R}), \psi_{\infty}},\left(\pi_{f}^{+}\right)^{\vee}\right)=0,
$$

since the Harish-Chandra parameter of $\left(\pi_{f}^{+}\right)^{\vee} \cong \pi_{f}^{-}$is $(-(2 \kappa+1))$ and $\mathrm{O}\left(V_{1}\right)(\mathbb{R}) \cong \mathrm{O}(3,1)$. Therefore, we have $\Phi=0$.

Next, we consider the case $B \neq 0$. By Lemma 5.1 (1), we have

$$
\mathcal{W}_{B}(g)=\int_{N\left(\mathbb{A}_{\mathbb{Q}}\right) \backslash \mathrm{SL}_{2}\left(\mathbb{A}_{\mathbb{Q}}\right)} \hat{\omega}\left(\alpha \cdot d\left(\nu\left(h_{g}\right)\right), h_{g}\right) \hat{\varphi}(-\beta ; 0,1) W_{\mathbf{f}, \operatorname{det}(B)}\left(\alpha \cdot d\left(\nu\left(h_{g}\right)\right)\right) d \alpha,
$$

where $h_{g}=\left(h_{g, v}\right)_{v} \in \operatorname{GSO}(V)\left(\mathbb{A}_{\mathbb{Q}}\right)$ is a representative of $\phi(g) \in \operatorname{PGSO}(V)\left(\mathbb{A}_{\mathbb{Q}}\right)$. We put $\xi=\operatorname{det}(B)$. If $\xi=0$, we find that $\mathcal{W}_{B}(g)=0$ since $\mathbf{f}$ is a cusp form. If $\xi \neq 0$, we find that $\mathcal{W}_{B}(g)=\prod_{v} \mathcal{W}_{B, v}\left(g_{v}\right)$, where for $g \in \mathrm{GU}(2,2)\left(\mathbb{Q}_{v}\right)$, we put

$$
\mathcal{W}_{B, v}(g)=\int_{N\left(\mathbb{Q}_{v}\right) \backslash \mathrm{SL}_{2}\left(\mathbb{Q}_{v}\right)} \hat{\omega}\left(\alpha \cdot d\left(\nu\left(h_{g}\right)\right), h_{g}\right) \hat{\varphi}_{v}(-\beta ; 0,1) W_{\mathbf{f}, v}\left(a(\xi) \cdot \alpha \cdot d\left(\nu\left(h_{g}\right)\right)\right) d \alpha .
$$

The following lemma will be proved in the last section. Here, we use the coordinate

$$
B=\left(\begin{array}{cc}
b_{1} & b_{2}+\sqrt{-D} b_{3} \\
b_{2}-\sqrt{-D} b_{3} & b_{4}
\end{array}\right) .
$$

Lemma 6.7. Fix $B \in \mathrm{Her}_{2}(\mathbb{Q})$.

(1) Let $x_{\infty}=\left(z \mathbf{1}_{4}\right) \cdot n(X) \cdot m(A) \cdot k \cdot r_{t} \in \mathrm{GU}(2,2)(\mathbb{R})^{+}$for $z \in \mathbb{C}^{\times}, X \in \operatorname{Her}_{2}(\mathbb{R})$, $A \in \mathrm{GL}_{2}(\mathbb{C})$ with $\operatorname{det}(A) \in \mathbb{R}^{\times}, t \in \mathbb{C}^{\times}$with $|t|=1$, and

$$
k=\left(\begin{array}{cc}
\alpha & \beta \\
-\beta & \alpha
\end{array}\right) \in \mathbb{K}_{0}=\mathbb{K} \cap \mathrm{SU}(2,2)(\mathbb{R}) .
$$

Put $Y=A^{t} \bar{A}$ and $Z=X+Y \sqrt{-1}$. Then, $\mathcal{W}_{B, \infty}\left(x_{\infty}\right)$ is equal to

$$
\begin{cases}2^{2 \kappa+2} \operatorname{det}(Y)^{\kappa+1} \xi^{\kappa+(1 / 2)} e^{2 \pi \sqrt{-1} \operatorname{Tr}(B Z)} \operatorname{det}(\alpha+\sqrt{-1} \beta)^{2 \kappa+2} & \text { if } B>0, \\ 0 & \text { otherwise. }\end{cases}
$$


(2) Assume that $p \nmid D$. We put

$$
m_{0}= \begin{cases}\min \left(\operatorname{ord}_{p}\left(b_{1}\right), \operatorname{ord}_{p}\left(b_{2}\right), \operatorname{ord}_{p}\left(b_{3}\right), \operatorname{ord}_{p}\left(b_{4}\right)\right) & \text { if } p \neq 2, \\ \min \left(\operatorname{ord}_{p}\left(b_{1}\right), \operatorname{ord}_{p}\left(b_{2}\right)+1, \operatorname{ord}_{p}\left(b_{3}\right)+1, \operatorname{ord}_{p}\left(b_{4}\right)\right) & \text { if } p=2 .\end{cases}
$$

Then for all $k \in \mathrm{GU}(2,2)\left(\mathbb{Z}_{p}\right)$, we have

$$
\mathcal{W}_{B, p}(k)=|\xi|_{p}^{\kappa+1 / 2} \sum_{n=0}^{m_{0}}\left(p^{n}\right)^{2 \kappa+1} a_{f}\left(\left(\xi p^{-2 n}\right)_{p}\right) .
$$

(3) Assume that $p \mid D$. We put

$$
m_{0}= \begin{cases}\min \left(\operatorname{ord}_{p}\left(b_{1}\right), \operatorname{ord}_{p}\left(b_{2}\right), \operatorname{ord}_{p}\left(b_{3}\right)+1, \operatorname{ord}_{p}\left(b_{4}\right)\right) & \text { if } \operatorname{ord}_{p}(D)=1, \\ \min \left(\operatorname{ord}_{p}\left(b_{1}\right), \operatorname{ord}_{p}\left(b_{2}\right)+1, \operatorname{ord}_{p}\left(b_{3}\right)+2, \operatorname{ord}_{p}\left(b_{4}\right)\right) & \text { if } \operatorname{ord}_{p}(D)=2, \\ \min \left(\operatorname{ord}_{p}\left(b_{1}\right), \operatorname{ord}_{p}\left(b_{2}\right)+1, \operatorname{ord}_{p}\left(b_{3}\right)+3, \operatorname{ord}_{p}\left(b_{4}\right)\right) & \text { if } \operatorname{ord}_{p}(D)=3 .\end{cases}
$$

Then for all $k \in \mathrm{GU}(2,2)\left(\mathbb{Z}_{p}\right)$, we have

$$
\mathcal{W}_{B, p}(k)=q_{p}^{-1} a_{f}\left(D_{p}\right)^{-1}|\xi|_{p}^{\kappa+1 / 2} \sum_{n=0}^{m_{0}}\left(p^{n}\right)^{2 \kappa+1} X_{n, p}
$$

where $q_{p}=\left(\mathrm{SL}_{2}\left(\mathbb{Z}_{p}\right): K_{0}\left(D ; \mathbb{Z}_{p}\right)\right)$ and

$$
X_{n, p}= \begin{cases}1 & \text { if }\left(D \xi p^{-2 n}\right)_{p}=1, \\ a_{f}\left(\left(D \xi p^{-2 n}\right)_{p}\right)+\underline{\chi}_{p}(-\xi) \overline{a_{f}\left(\left(D \xi p^{-2 n}\right)_{p}\right)} & \text { otherwise. }\end{cases}
$$

(4) Assume that $\operatorname{ord}_{p}\left(C_{i}\right) \neq 0$. Write $t_{i, p}=x_{p} \otimes 1+y_{p} \otimes \sqrt{-D} \in \mathbb{Q}_{p} \otimes K$. Then for all $k \in \mathrm{GU}(2,2)\left(\mathbb{Z}_{p}\right)$, we have

$$
\mathcal{W}_{B, p}\left(\gamma_{i, p} k\right)=\left|C_{i}\right|_{p}^{\kappa+1}|\xi|_{p}^{\kappa+1 / 2} \sum_{n=0}^{\infty} \hat{\varphi}_{p}\left(-\phi^{\prime}\left(t_{i, p}\right) \beta p^{-n} ; 0, p^{n}\right)\left(p^{n}\right)^{2 \kappa+1} a_{f}\left(\left(\xi C_{i} p^{-2 n}\right)_{p}\right) \text {. }
$$

Here $\phi^{\prime}\left(t_{i, p}\right) \in \mathrm{GL}\left(V_{1}\right) \cong \mathrm{GL}_{4}\left(\mathbb{Q}_{p}\right)$ is given by

$$
\phi^{\prime}\left(t_{i, p}\right)=\left(\begin{array}{cccc}
N_{K_{p} / \mathbb{Q}_{p}}\left(t_{i, p}\right) & & & \\
& x_{p} & -D y_{p} & \\
& y_{p} & x_{p} & \\
& & & 1
\end{array}\right),
$$

which makes the following diagram commutative.

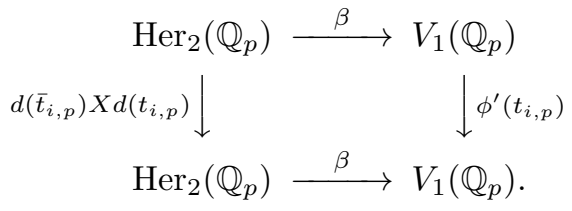

6.5. Proof of Proposition 6.2. The ring of integers $\mathfrak{o}$ of $K=\mathbb{Q}(\sqrt{-D})$ is given by

$$
\mathfrak{o}= \begin{cases}\mathbb{Z}+\frac{1+\sqrt{-D}}{2} \mathbb{Z} & \text { if } D \text { is odd } \\ \mathbb{Z}+\frac{\sqrt{-D}}{2} \mathbb{Z} & \text { if } D \text { is even. }\end{cases}
$$

So we change the coordinate $B=B\left(h_{1}, \ldots, h_{4}\right)$ of $\operatorname{Her}_{2}\left(\mathbb{A}_{\mathbb{Q}}\right)$ such that $d\left(\bar{t}_{i}\right) B d\left(t_{i}\right)$ is equal to

$$
\left\{\begin{array}{cc}
\left(\begin{array}{cc}
h_{1} & \left(h_{2}+h_{3} / 2\right) / \sqrt{-D}+h_{3} / 2 \\
-\left(h_{2}+h_{3} / 2\right) / \sqrt{-D}+h_{3} / 2 & h_{4}
\end{array}\right) & \text { if } D \text { is odd, } \\
\left(\begin{array}{cc}
h_{1} & h_{2} / \sqrt{-D}+h_{3} / 2 \\
-h_{2} / \sqrt{-D}+h_{3} / 2 & h_{4}
\end{array}\right) & \text { if } D \text { is even }
\end{array}\right.
$$


for $h_{1}, \ldots, h_{4} \in \mathbb{A}_{\mathbb{Q}}$. Note that $B \in \Lambda_{2}^{\mathfrak{c}_{i}}(\mathfrak{o})$ if and only if $\operatorname{ord}_{p}\left(h_{i}\right) \geq 0$ for all $i=1, \ldots, 4$ and all prime $p$.

Lemma 6.8. We put $m_{i}=\operatorname{ord}_{p}\left(b_{i}\right)$ and $l_{i}=\operatorname{ord}_{p}\left(h_{i}\right)$.

(1) If $p \nmid 2 D C_{i}$, then we have

$$
\min \left(m_{1}, m_{2}, m_{3}, m_{4}\right)=\min \left(l_{1}, l_{2}, l_{3}, l_{4}\right) .
$$

(2) If $p \neq 2$ and $p \mid D$, then we have

$$
\min \left(m_{1}, m_{2}, m_{3}+1, m_{4}\right)=\min \left(l_{1}, l_{2}, l_{3}, l_{4}\right) .
$$

(3) Let $p=2 \nmid D$. Unless $l_{2}<\min \left(l_{1}, l_{3}, l_{4}\right)$, we have

$$
\min \left(m_{1}, m_{2}+1, m_{3}+1, m_{4}\right)=\min \left(l_{1}, l_{2}, l_{3}, l_{4}\right) .
$$

In this case, we have

$$
\min \left(m_{1}, m_{2}+1, m_{3}+1, m_{4}\right)=l_{2}+1, \quad \min \left(l_{1}, l_{2}, l_{3}, l_{4}\right)=l_{2},
$$

and we have $\xi / 2^{2\left(l_{2}+1\right)} \notin \mathbb{Z}_{2}$.

(4) If $p=2 \mid D$, then we have

$$
\min \left(m_{1}, m_{2}+1, m_{3}+\operatorname{ord}_{2}(D), m_{4}\right)=\min \left(l_{1}, l_{2}, l_{3}, l_{4}\right) .
$$

(5) If $p \mid C_{i}$, we have

$$
\sum_{n=0}^{\infty} \hat{\varphi}_{p}\left(-\phi^{\prime}\left(t_{i, p}\right) \beta p^{-n} ; 0, p^{n}\right)\left(p^{n}\right)^{2 \kappa+1} a_{f}\left(\left(\xi C p^{-2 n}\right)_{p}\right)=\sum_{n=0}^{\min \left(l_{i}\right)}\left(p^{n}\right)^{2 \kappa+1} a_{f}\left(\left(\xi C p^{-2 n}\right)_{p}\right) \text {. }
$$

Proof. (5) is easily followed from the last assertion of Lemma 6.7 (4). If $D$ is even, the assertions (1) to (4) are obvious. So we assume $D$ is odd.

If $p \nmid 2 D C_{i}$, then we have $m_{1}=l_{1}, m_{2}=l_{3}, m_{4}=l_{4}$ and $m_{3}=\operatorname{ord}_{p}\left(h_{2}+h_{3} / 2\right)$. If $l_{2} \geq l_{3}$, then we have

Hence we have

$$
m_{3}=\operatorname{ord}_{p}\left(h_{2}+h_{3} / 2\right) \geq l_{3}=m_{2} .
$$

$$
\min \left(m_{1}, m_{2}, m_{3}, m_{4}\right)=\min \left(m_{1}, m_{2}, m_{4}\right)=\min \left(l_{1}, l_{3}, l_{4}\right)=\min \left(l_{1}, l_{2}, l_{3}, l_{4}\right) .
$$

If $l_{2}<l_{3}$, then we have $m_{3}=l_{2}$. So we have (1).

If $p \neq 2$ and $p \mid D$, then we have $m_{1}=l_{1}, m_{2}=l_{3}, m_{4}=l_{4}$ and $m_{3}+1=\operatorname{ord}_{p}\left(h_{2}+h_{3} / 2\right)$. Then, the proof of (2) is similar to that of (1).

If $p=2 \nmid D$, then we have $m_{1}=l_{1}, m_{2}+1=l_{3}, m_{4}=l_{4}$ and $m_{3}+1=\operatorname{ord}_{p}\left(2 h_{2}+h_{3}\right)$. If $l_{2}+1>l_{3}$, then we have

Hence we have

$$
m_{3}+1=l_{3}=m_{2}+1 \text {. }
$$

$$
\begin{aligned}
\min \left(m_{1}, m_{2}+1, m_{3}+1, m_{4}\right) & =\min \left(m_{1}, m_{2}+1, m_{4}\right) \\
& =\min \left(l_{1}, l_{3}, l_{4}\right)=\min \left(l_{1}, l_{2}, l_{3}, l_{4}\right) .
\end{aligned}
$$

If $l_{2}+1<l_{3}$, then we have

$$
m_{3}+1=l_{2}+1<m_{2}+1 \text {. }
$$

Hence we have

$$
\begin{aligned}
\min \left(m_{1}, m_{2}+1, m_{3}+1, m_{4}\right) & =\min \left(m_{1}, m_{3}+1, m_{4}\right)=\min \left(l_{1}, l_{2}+1, l_{4}\right), \\
\min \left(l_{1}, l_{2}, l_{3}, l_{4}\right) & =\min \left(l_{1}, l_{2}, l_{4}\right) .
\end{aligned}
$$

So if

$$
\min \left(m_{1}, m_{2}+1, m_{3}+1, m_{4}\right) \neq \min \left(l_{1}, l_{2}, l_{3}, l_{4}\right),
$$

then we have $l_{2}<\min \left(l_{1}, l_{4}\right)$ and

$$
\min \left(m_{1}, m_{2}+1, m_{3}+1, m_{4}\right)=l_{2}+1 \quad \text { and } \min \left(l_{1}, l_{2}, l_{3}, l_{4}\right)=l_{2} .
$$


In this case, we have $l_{1}+l_{4}>2 l_{2}, 2 l_{3}-2>2 l_{2}$, and $\operatorname{ord}_{2}\left(h_{2}+h_{3} / 2\right)^{2}=2 l_{2}$. Now, we find that

$$
\xi=\operatorname{det}(H)=h_{1} h_{4}-\frac{h_{3}^{2}}{4}-\frac{1}{D}\left(h_{2}+\frac{h_{3}}{2}\right)^{2} .
$$

Hence we have $\operatorname{ord}_{2}(\xi)=2 l_{2}<2\left(l_{2}+1\right)$. If $l_{2}+1=l_{3}$, then we find $m_{3}+1 \geq l_{2}+1$ and $l_{2}<l_{3}$. So we have

$$
\begin{aligned}
\min \left(m_{1}, m_{2}+1, m_{3}+1, m_{4}\right) & =\min \left(l_{1}, l_{2}+1, l_{4}\right), \\
\min \left(l_{1}, l_{2}, l_{3}, l_{4}\right) & =\min \left(l_{1}, l_{2}, l_{4}\right) .
\end{aligned}
$$

So if

$$
\min \left(m_{1}, m_{2}+1, m_{3}+1, m_{4}\right) \neq \min \left(l_{1}, l_{2}, l_{3}, l_{4}\right),
$$

then we have $l_{2}<\min \left(l_{1}, l_{4}\right)$ and

$$
\min \left(m_{1}, m_{2}+1, m_{3}+1, m_{4}\right)=l_{2}+1 \quad \text { and } \min \left(l_{1}, l_{2}, l_{3}, l_{4}\right)=l_{2} .
$$

Since the rational numbers $h_{2}, h_{3}$ satisfy that $\operatorname{ord}_{2}\left(h_{2}\right)=\operatorname{ord}_{2}\left(h_{3} / 2\right)$, we have

$$
\operatorname{ord}_{2}\left(h_{2}+h_{3} / 2\right)>\operatorname{ord}_{2}\left(h_{2}\right)=l_{2} .
$$

However we have $\operatorname{ord}_{2}\left(h_{1} h_{4}\right)=l_{1}+l_{4}>2 l_{2}$ and $\operatorname{ord}_{2}\left(h_{3}^{2} / 4\right)=2 l_{3}-2=2 l_{2}$. Hence we have $\operatorname{ord}_{2}(\xi)=2 l_{2}<2\left(l_{2}+1\right)$. We get (3). This completes the proof.

Now, we start to prove Proposition 6.2. By Sect. 6.3 and Lemma 6.7, the compact group $\mathbb{K} \cdot \mathcal{K}_{0} \subset \mathrm{U}(2,2)\left(\mathbb{A}_{\mathbb{Q}}\right)$ acts on $\mathbf{F}=$ Lift $^{(2)}(f)$ and on $\theta(\mathbf{f}, \varphi)$ by the same character. Hence, it suffices to show that

$$
\mathcal{W}_{B}\left(\gamma_{i} x_{\infty}\right)=\left(\prod_{p \mid D} q_{p}^{-1}\right) 2^{2 \kappa+2} a_{f}(D)^{-1} \mathcal{W}_{\mathbf{F}, B}\left(\gamma_{i} x_{\infty}\right)
$$

for all $B \in \operatorname{Her}_{2}(\mathbb{Q})$ and $x_{\infty}=n(X) \cdot m(A)$, where $X \in \operatorname{Her}_{2}(\mathbb{R}), A \in \mathrm{GL}_{2}(\mathbb{C})$ with $\operatorname{det}(A) \in \mathbb{R}^{\times}$ and $q_{p}=\left(\mathrm{SL}_{2}\left(\mathbb{Z}_{p}\right): K_{0}\left(D ; \mathbb{Z}_{p}\right)\right)$. We may assume that $B \in \Lambda_{2}^{\mathfrak{c}_{i}}(\mathfrak{o})^{+}$. Then $\mathcal{W}_{B}\left(\gamma_{i} x_{\infty}\right)$ is equal to

$$
\begin{aligned}
& 2^{2 \kappa+2} \operatorname{det}(Y)^{\kappa+1} e^{2 \pi \sqrt{-1} \operatorname{Tr}(B Z)} C_{i}^{-\kappa-1}\left(\prod_{p \mid D} q_{p}^{-1}\right) a_{f}(D)^{-1} \\
& \times\left(\prod_{p \nmid D} \sum_{n=0}^{\min \left(l_{i}\right)}\left(p^{n}\right)^{2 \kappa+1} a_{f}\left(\left(\xi C_{i} p^{-2 n}\right)_{p}\right)\right)\left(\prod_{p \mid D} \sum_{n=0}^{\min \left(l_{i}\right)}\left(p^{n}\right)^{2 \kappa+1} X_{n, p}\right) \\
& =2^{2 \kappa+2} \operatorname{det}(Y)^{\kappa+1} e^{2 \pi \sqrt{-1} \operatorname{Tr}(B Z)} C_{i}^{-\kappa-1}\left(\prod_{p \mid D} q_{p}^{-1}\right) a_{f}(D)^{-1} \\
& \times \sum_{d \mid\left(h_{1}, h_{2}, h_{3}, h_{4}\right)} d^{2 \kappa+1} \alpha_{F_{\mathrm{c}_{i}}}\left(\frac{C_{i} D \operatorname{det}(B)}{d^{2}}\right) \\
& =2^{2 \kappa+2}\left(\prod_{p \mid D} q_{p}^{-1}\right) a_{f}(D)^{-1} \mathcal{W}_{\mathbf{F}, B}\left(\gamma_{i} x_{\infty}\right) .
\end{aligned}
$$

This completes the proof of Proposition 6.2 using Lemma 6.7.

Corollary 6.9. The automorphic form Lift ${ }^{(2)}(f)$ on $\mathrm{U}(2,2)\left(\mathbb{A}_{\mathbb{Q}}\right)$ can be extended to $\mathrm{GU}(2,2)\left(\mathbb{A}_{\mathbb{Q}}\right)$ by

$$
\operatorname{Lift}^{(2)}(f)\left(u r_{t} x k\right)=C^{-\kappa-1}\left(F_{\mathfrak{c}} \|_{2 \kappa+2} x\right)(\mathbf{i})=C^{-\kappa-1} F_{\mathfrak{c}}(x\langle\mathbf{i}\rangle) j(x, \mathbf{i})^{-2 \kappa-2}(\operatorname{det} x)^{\kappa+1}
$$


for all $u \in \mathrm{GU}(2,2)(\mathbb{Q}), x \in \mathrm{GU}(2,2)(\mathbb{R})^{+}, k \in \mathcal{K}$ and $t \in \mathbb{A}_{K, \text { fin }}$ such that the ideal $\mathfrak{c}$ given by $t$ is in $J_{K}^{D}$. Here, $C=N(\mathfrak{c}) \in \mathbb{Q}_{>0}$ is the ideal norm of $\mathfrak{c}$. Moreover, it satisfies

$$
\theta(\phi(g) ; \mathbf{f}, \varphi)=\left(\prod_{p \mid D} q_{p}^{-1}\right) 2^{2 \kappa+2} a_{f}(D)^{-1} \operatorname{Lift}^{(2)}(f)(g)
$$

for all $g \in \mathrm{GU}(2,2)\left(\mathbb{A}_{\mathbb{Q}}\right)$ with $q_{p}=\left(\mathrm{SL}_{2}\left(\mathbb{Z}_{p}\right): K_{0}\left(D ; \mathbb{Z}_{p}\right)\right)$.

Proof. This follows from Proposition 6.2 and Lemma 2.2.

\section{ThetA CORRESPONDENCE FOR $\left(\mathrm{GL}_{2}, \mathrm{GO}(2,2)\right)$}

In this section, we recall the theta correspondence for $\left(\mathrm{GL}_{2}, \mathrm{GO}(2,2)\right)$ following [14] §6. For the proof of Theorem 3.2, we need Lemma 7.1 which calculates a certain theta lift on $G_{2}\left(\mathbb{A}_{\mathbb{Q}}\right)$.

Let $\mathrm{M}_{2}$ be the vector space of $2 \times 2$ matrices. We regard $\mathrm{M}_{2}$ as the quadratic space with the quadratic form

$$
Q[x]=\operatorname{det}(x) .
$$

Let

$$
\mathrm{G}\left(\mathrm{SL}_{2} \times \mathrm{SL}_{2}\right)=\left\{\left(h_{1}, h_{2}\right) \in \mathrm{GL}_{2} \times \mathrm{GL}_{2} \mid \operatorname{det}\left(h_{1}\right)=\operatorname{det}\left(h_{2}\right)\right\} .
$$

For $\left(h_{1}, h_{2}\right) \in \mathrm{G}\left(\mathrm{SL}_{2} \times \mathrm{SL}_{2}\right)$, we put $\Delta\left(h_{1}, h_{2}\right)=\operatorname{det}\left(h_{1}\right)=\operatorname{det}\left(h_{2}\right)$. Recall that there is a following exact commutative diagram:

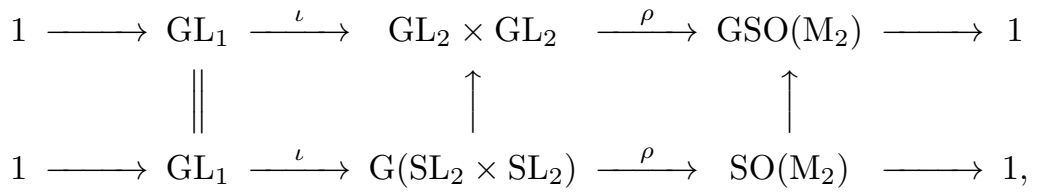

where

$$
\iota(a)=\left(a \mathbf{1}_{2}, a \mathbf{1}_{2}\right) \quad \text { and } \quad \rho\left(h_{1}, h_{2}\right) x=h_{1} x h_{2}^{-1},
$$

for $a \in \mathrm{GL}_{1}, h_{1}, h_{2} \in \mathrm{GL}_{2}$ and $x \in \mathrm{M}_{2}$.

Let $V^{\prime}$ be the subspace of $V$ generated by $\left\{e_{1}, e_{2}, e_{5}, e_{6}\right\}$. Then there is an isomorphism of quadratic spaces over $\mathbb{Q}$ as follows:

$$
V^{\prime}(\mathbb{Q}) \stackrel{\cong}{\rightarrow} \mathrm{M}_{2}(\mathbb{Q}), \quad x_{1} e_{1}+x_{2} e_{2}+x_{5} e_{5}+x_{6} e_{6} \mapsto\left(\begin{array}{cc}
x_{2} & -x_{1} \\
x_{6} & x_{5}
\end{array}\right) .
$$

Via this isomorphism, we regard $\rho$ as a map $\rho: \mathrm{GL}_{2} \times \mathrm{GL}_{2} \rightarrow \mathrm{GSO}\left(V^{\prime}\right)$.

Let $\mu_{2}$ be the subgroup of $\mathrm{O}\left(\mathrm{M}_{2}\right)$ generated be the involution $*$ on $\mathrm{M}_{2}$ given by

$$
x=\left(\begin{array}{ll}
x_{1} & x_{2} \\
x_{3} & x_{4}
\end{array}\right) \mapsto x^{*}=\left(\begin{array}{cc}
x_{4} & -x_{2} \\
-x_{3} & x_{1}
\end{array}\right) .
$$

Via the isomorphism $\mathrm{M}_{2} \cong V^{\prime}$, the involution $*$ on $\mathrm{M}_{2}$ corresponds to an element $h_{0}^{\prime} \in \mathrm{O}\left(V^{\prime}\right)$ given by

$$
x_{1} \mapsto-x_{1}, x_{2} \mapsto x_{5}, x_{5} \mapsto x_{2}, x_{6} \mapsto-x_{6} .
$$

For $\alpha \in K^{\times}$, we have $\phi_{2}(\alpha) V^{\prime} \subset V^{\prime}$. We put $\phi_{2}^{\prime}(\alpha)=\left.\phi_{2}(\alpha)\right|_{V^{\prime}}$ for $\alpha \in K^{\times}$. Then we find that

$$
\phi_{2}^{\prime}(\alpha)=\left(\begin{array}{cccc}
N_{K / \mathbb{Q}}(\alpha) & & & \\
& 1 & & \\
& & N_{K / \mathbb{Q}}(\alpha) & \\
& & & 1
\end{array}\right)=\rho\left(\mathbf{1}_{2}, d\left(N_{K / \mathbb{Q}}(\alpha)^{-1}\right)\right) \in \operatorname{GSO}\left(V^{\prime}\right) .
$$


We extend $g \in \mathrm{SO}\left(V^{\prime}\right)$ to $\mathrm{SO}(V)$ by $g e_{3}=e_{3}$ and $g e_{4}=e_{4}$. We define the inclusion $\tau: \mathrm{G}\left(\mathrm{SL}_{2} \times\right.$ $\left.\mathrm{SL}_{2}\right) \hookrightarrow \mathrm{GSU}(2,2)$ by

$$
\tau\left(\left(\begin{array}{ll}
a_{1} & b_{1} \\
c_{1} & d_{1}
\end{array}\right),\left(\begin{array}{ll}
a_{2} & b_{2} \\
c_{2} & d_{2}
\end{array}\right)\right) \mapsto\left(\begin{array}{cccc}
a_{1} & 0 & b_{1} & 0 \\
0 & a_{2} & 0 & b_{2} \\
c_{1} & 0 & d_{1} & 0 \\
0 & c_{2} & 0 & d_{2}
\end{array}\right) .
$$

Then we find that the following diagram is commutative:

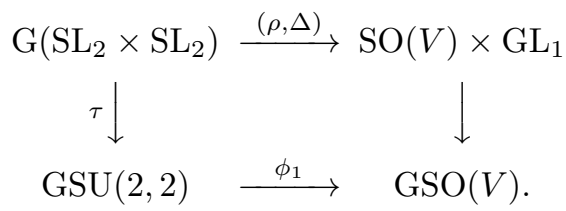

Here, the map $\mathrm{SO}(V) \times \mathrm{GL}_{1} \rightarrow \mathrm{GSO}(V)$ is the multiplication $(h, a) \mapsto a h$.

For a normalized Hecke eigenform $g \in S_{2 \kappa+2}\left(\mathrm{SL}_{2}(\mathbb{Z})\right)$, let $\mathbf{g}$ denote the cusp form on $\mathrm{GL}_{2}\left(\mathbb{A}_{\mathbb{Q}}\right)$ given by $g$. Following [14] $\S 6.3$, we extend the cusp form $\mathbf{g} \otimes \mathbf{g}$ on $\mathrm{GSO}\left(\mathrm{M}_{2}\right)\left(\mathbb{A}_{\mathbb{Q}}\right)$ to a cusp form $\mathcal{G}$ on $\mathrm{GO}\left(\mathrm{M}_{2}\right)\left(\mathbb{A}_{\mathbb{Q}}\right)$ by $\mathcal{G}\left(h h^{\prime}\right)=\mathcal{G}(h)$ for $h \in \mathrm{GO}\left(\mathrm{M}_{2}\right)\left(\mathbb{A}_{\mathbb{Q}}\right)$ and $h^{\prime} \in \mu_{2}\left(\mathbb{A}_{\mathbb{Q}}\right)$.

We define $\varphi^{\prime}=\otimes \varphi_{v}^{\prime} \in \mathcal{S}\left(V^{\prime}\left(\mathbb{A}_{\mathbb{Q}}\right)\right)$ as follows:

- If $v=p<\infty$, then $\varphi_{p}^{\prime}$ is the characteristic function of $V^{\prime}\left(\mathbb{Z}_{p}\right)$.

- If $v=\infty$, then

$$
\varphi_{\infty}^{\prime}\left(x_{1} e_{1}+x_{2} e_{2}+x_{5} e_{5}+x_{6} e_{6}\right)=\left(-\sqrt{-1} x_{1}+x_{2}-x_{5}+\sqrt{-1} x_{6}\right)^{2 \kappa+2} e^{-\pi\left(x_{1}^{2}+x_{2}^{2}+x_{5}^{2}+x_{6}^{2}\right)} .
$$

We may regard $\mathcal{G}$ as a cusp form on $\operatorname{GO}\left(V^{\prime}\right)\left(\mathbb{A}_{\mathbb{Q}}\right)$ via the isomorphism $\mathrm{M}_{2} \cong V^{\prime}$. So we can consider the theta lift $\theta\left(\overline{\mathcal{G}}, \varphi^{\prime}\right)$ on $\mathrm{GL}_{2}\left(\mathbb{A}_{\mathbb{Q}}\right)$. The following lemma is Lemma 6.3 in [14].

Lemma 7.1. For all $\alpha \in \mathrm{GL}_{2}\left(\mathbb{A}_{\mathbb{Q}}\right)$, we have

$$
\theta\left(\alpha ; \overline{\mathcal{G}}, \varphi^{\prime}\right)=2^{2 \kappa+1} \xi_{\mathbb{Q}}(2)^{-2}\langle g, g\rangle \overline{\mathbf{g}(\alpha)} .
$$

\section{Theta correspondence for $\left(\mathrm{SL}_{2}, \mathrm{O}(2)\right)$ and the Siegel-Weil formula}

In this section, we study the theta correspondence for $\left(\mathrm{SL}_{2}, \mathrm{O}(2)\right)$. For the proof of Theorem 3.2 , we need the Siegel-Weil formula (Proposition 8.1) which calculates a certain theta lift on $\mathrm{SL}_{2}\left(\mathbb{A}_{\mathbb{Q}}\right)$.

We regard $K$ as the quadratic space over $\mathbb{Q}$ with the bilinear form

$$
(x, y)=\operatorname{Tr}_{K / \mathbb{Q}}(x \bar{y}) .
$$

Then the associated quadratic form is given by

$$
Q[x]=N_{K / \mathbb{Q}}(x) .
$$

Let $V^{\prime \prime}$ be the subspace of $V$ generated by $\left\{e_{3}, e_{4}\right\}$. Then there is an isomorphism of quadratic spaces over $\mathbb{Q}$ as follows:

$$
\ell: K \stackrel{\cong}{\rightarrow} V^{\prime \prime}, x+y \sqrt{-D} \mapsto x e_{3}+y e_{4} .
$$

This isomorphism induces an isomorphism of algebraic groups as follows:

$$
K^{\times} \cong \mathrm{GSO}\left(V^{\prime \prime}\right) \cong \mathrm{GSO}(2), x+y \sqrt{-D} \mapsto\left(\begin{array}{cc}
x & -D y \\
y & x
\end{array}\right) .
$$

The restriction of this map gives an isomorphism $K^{1} \cong \mathrm{SO}\left(V^{\prime \prime}\right)$.

For $\alpha \in K^{\times}$, we have $\phi_{2}(\alpha) V^{\prime \prime} \subset V^{\prime \prime}$. We put $\phi_{2}^{\prime \prime}(\alpha)=\left.\phi_{2}(\alpha)\right|_{V^{\prime \prime}}$. Then for $\alpha \in K^{\times}$and $x \in K$, we have

$$
\phi_{2}^{\prime \prime}(\alpha) \ell(x)=\ell(\bar{\alpha} x)
$$


i.e., the following diagram is commutative:

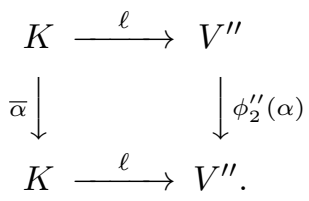

For $\varphi^{\prime \prime} \in \mathcal{S}\left(V^{\prime \prime}\left(\mathbb{A}_{\mathbb{Q}}\right)\right)$, we define

$$
\Phi(\alpha, s)=\Phi\left(\alpha, s, \varphi^{\prime \prime}\right)=\left[\omega(\alpha, 1) \varphi^{\prime \prime}\right](0)|a(\alpha)|^{s}
$$

where if $\alpha=n(x) t(a) k \in \mathrm{SL}_{2}\left(\mathbb{A}_{\mathbb{Q}}\right)$ with $x \in \mathbb{A}_{\mathbb{Q}}, a \in \mathbb{A}_{\mathbb{Q}}^{\times}$and $k \in \mathrm{SL}_{2}(\hat{\mathbb{Z}}) \operatorname{SO}(2)$, then we put

$$
|a(\alpha)|=|a| \text {. }
$$

Note that the quantity $|a(\alpha)|$ is well-defined. It satisfies that

$$
\Phi(n(x) t(a) g, s)=\chi_{V^{\prime \prime}}(a)|a|^{s+1} \Phi(g, s)
$$

for all $x \in \mathbb{A}_{\mathbb{Q}}, a \in \mathbb{A}_{\mathbb{Q}}^{\times}$and $g \in \operatorname{SL}_{2}\left(\mathbb{A}_{\mathbb{Q}}\right)$, i.e., $\Phi \in \operatorname{Ind}_{B\left(\mathbb{A}_{\mathbb{Q}}\right)}^{\mathrm{SL}_{2}\left(\mathbb{A}_{\mathbb{Q}}\right)}\left(\chi_{V^{\prime \prime}}|\cdot|^{s}\right)$. Note that $\Phi(\alpha, s)=$ $\prod_{v} \Phi_{v}\left(\alpha_{v}, s\right)$.

We define the Eisenstein series $E(\alpha, s)$ by

$$
E(\alpha, s)=\sum_{\gamma \in B(\mathbb{Q}) \backslash \mathrm{SL}_{2}(\mathbb{Q})} \Phi(\gamma \alpha, s) .
$$

This is absolutely convergent for $\operatorname{Re}(s)>1$. The Eisenstein series $E(\alpha, s)$ has a meromorphic continuation to the whole $s$-plane and is holomorphic at $s=0$. The following proposition is Main Theorem of [19].

Proposition 8.1 (Siegel-Weil formula). For $\varphi^{\prime \prime} \in \mathcal{S}\left(V^{\prime \prime}\left(\mathbb{A}_{\mathbb{Q}}\right)\right)$, we have

$$
\theta\left(\alpha ; \mathbf{1}_{\mathrm{O}\left(V^{\prime \prime}\right)\left(\mathbb{A}_{\mathbb{Q}}\right)}, \varphi^{\prime \prime}\right)=\frac{1}{2} E(\alpha, 0) .
$$

We define $\varphi^{\prime \prime}=\otimes_{v} \varphi_{v}^{\prime \prime} \in \mathcal{S}\left(V^{\prime \prime}\left(\mathbb{A}_{\mathbb{Q}}\right)\right)$ as follows:

- If $v=p<\infty$ and $p \neq 2$, then $\varphi_{p}^{\prime \prime}$ is the characteristic function of $V^{\prime \prime}\left(\mathbb{Z}_{p}\right)$.

- If $v=2$, then $\varphi_{2}^{\prime \prime}$ is the characteristic function of

$$
\begin{cases}V^{\prime \prime}\left(\mathbb{Z}_{2}\right) \cup\left(2^{-1} \mathbb{Z}_{2}^{\times} e_{3}+2^{-1} \mathbb{Z}_{2}^{\times} e_{4}\right) & \text { if } D \text { is odd } \\ \mathbb{Z}_{2} e_{3}+2^{-1} \mathbb{Z}_{2} e_{4} & \text { if } D \text { is even. }\end{cases}
$$

- If $v=\infty$, then

$$
\varphi_{\infty}^{\prime \prime}(x)=e^{-2 \pi Q^{\prime \prime}[x]}
$$

Here, $Q^{\prime \prime}$ is the quadratic form of $V^{\prime \prime}$ given by

$$
Q^{\prime \prime}\left[x_{3} e_{3}+x_{4} e_{4}\right]=x_{3}^{2}+D x_{4}^{2} .
$$

We will apply Proposition 8.1 to $L\left(\phi_{2}^{\prime \prime}(t)\right) \varphi^{\prime \prime}$ for some $t \in \mathbb{A}_{K, \text { fin }}^{\times}$.

Lemma 8.2. Via the isomorphism $\ell: K \cong V^{\prime \prime}$, the function $\varphi_{p}^{\prime \prime}$ is the characteristic function of the maximal compact subring $\mathfrak{o} \otimes_{\mathbb{Z}} \mathbb{Z}_{p}$ of $K \otimes \mathbb{Q}_{p}$ for each $p$. In particular, for $\alpha \in\left(\mathfrak{o} \otimes_{\mathbb{Z}} \mathbb{Z}_{p}\right)^{\times}$, we have

$$
L\left(\phi_{2}^{\prime \prime}(\alpha)\right) \varphi_{p}^{\prime \prime}=\varphi_{p}^{\prime \prime}
$$

Proof. This is obvious.

We define

$$
\hat{\mathfrak{o}}_{K}^{\times}=\prod_{\mathfrak{p}} \mathfrak{o}_{K_{\mathfrak{p}}}^{\times}, \quad \hat{\mathfrak{o}}_{K}^{1}=\left\{\beta \bar{\beta}^{-1} \mid \beta \in \hat{\mathfrak{o}}_{K}^{\times}\right\}
$$

and

$$
\mathbb{C}^{1}=K^{1}(\mathbb{R})=\left\{x+y \sqrt{-D} \in \mathbb{C} \mid x, y \in \mathbb{R}, x^{2}+D y^{2}=1\right\}
$$


Note that $\mathbb{C}^{1}=\left\{\alpha \in \mathbb{C}^{\times}|| \alpha \mid=1\right\}$. We easily find that $L\left(\phi_{2}^{\prime \prime}(\alpha)\right) \varphi_{\infty}^{\prime \prime}=\varphi_{\infty}^{\prime \prime}$ for $\alpha \in \mathbb{C}^{1}$. This fact and Lemma 8.2 imply that the function

$$
\alpha \mapsto \theta\left(g, \phi_{2}^{\prime \prime}(\alpha) ; \varphi^{\prime \prime}\right)
$$

on $\left[K^{1}\right]$ is right $\hat{\mathfrak{o}}_{K}^{1} \mathbb{C}^{1}$-invariant for all $g \in \mathrm{SL}_{2}\left(\mathbb{A}_{\mathbb{Q}}\right)$.

\section{Proof of Theorem 3.2}

In this section, we prove Theorem 3.2 using Lemma 6.7 and Lemma 9.6, which will be proved in the last section.

9.1. Seesaw identity. Let $\mathfrak{c} \in J_{\mathfrak{o}}^{D}$ and put $C=N(\mathfrak{c}) \in \mathbb{Z}_{>0}$. Assume that $C$ is a square free integer. Let $t \in \mathbb{A}_{K, \text { fin }}^{\times}$such that $\operatorname{ord}_{\mathfrak{p}}\left(t_{\mathfrak{p}}\right)=\operatorname{ord}_{\mathfrak{p}}(\mathfrak{c})$ for each prime ideal $\mathfrak{p}$ of $K$. Note that $C N_{K / \mathbb{Q}}\left(t_{p}\right)^{-1} \in \mathbb{Z}_{p}^{\times}$for each $p$. We consider the integral

$$
I(\mathfrak{c})=\int_{\left[\mathrm{O}\left(V^{\prime}\right)\right]} \int_{\left[\mathrm{O}\left(V^{\prime \prime}\right)\right]} \theta\left(h^{\prime} h^{\prime \prime} \phi_{2}(t) ; \mathbf{f}, \varphi\right) \overline{\mathcal{G}\left(h^{\prime} \phi_{2}^{\prime}(t)\right)} d h^{\prime \prime} d h^{\prime} .
$$

Note that this integral does not depend on the choice of $t \in \mathbb{A}_{K, \text { fin }}^{\times}$. First, using Proposition 6.2, we get the following expression of $I(\mathfrak{c})$ in terms of the period integrals.

Proposition 9.1. The integral $I(\mathfrak{c})$ is equal to

$$
2^{-1} \xi_{\mathbb{Q}}(2)^{-2}\left(\prod_{p \mid D} q_{p}^{-1}\right) 2^{2 \kappa+1} a_{f}(D)^{-1} \frac{1}{\#\left(C l_{K}^{2}\right)} \sum_{[\mathfrak{a}] \in C l_{K}^{2}} \frac{\left\langle\left. F_{\mathfrak{a c}}\right|_{\mathfrak{H} \times \mathfrak{H}}, g \times g_{A C}\right\rangle\langle g, g\rangle}{\left\langle g_{A C}, g_{A C}\right\rangle} .
$$

Here, we put $q_{p}=\left(\mathrm{SL}_{2}\left(\mathbb{Z}_{p}\right): K_{0}\left(D ; \mathbb{Z}_{p}\right)\right), A=N(\mathfrak{a})$ and $C l_{K}^{2}=\left\{[\mathfrak{b}]^{2} \mid[\mathfrak{b}] \in C l_{K}\right\}$.

Proof. We define $h_{0}^{\prime} \in \mathrm{O}\left(V^{\prime}\right)$ by

$$
x_{1} \mapsto-x_{1}, x_{2} \mapsto x_{5}, x_{5} \mapsto x_{2}, x_{6} \mapsto-x_{6},
$$

and $h_{0}^{\prime \prime} \in \mathrm{O}\left(V^{\prime \prime}\right)$ by

$$
x_{3} \mapsto-x_{3}, x_{4} \mapsto x_{4} .
$$

We put $h_{1}^{\prime}=\phi_{2}^{\prime}(t) h_{0}^{\prime} \phi_{2}^{\prime}(t)^{-1}, h_{1}^{\prime \prime}=\phi_{2}^{\prime \prime}(t) h_{0}^{\prime \prime} \phi_{2}^{\prime \prime}(t)^{-1}$. Then we find that

$$
\theta\left(h^{\prime} h_{1}^{\prime} h^{\prime \prime} h_{1}^{\prime \prime} \phi_{2}(t) ; \mathbf{f}, \varphi\right) \overline{\mathcal{G}\left(h^{\prime} h_{1}^{\prime} \phi_{2}^{\prime}(t)\right)}=\theta\left(h^{\prime} h^{\prime \prime} \phi_{2}(t) ; \mathbf{f}, \varphi\right) \overline{\mathcal{G}\left(h^{\prime} \phi_{2}^{\prime}(t)\right)}
$$

for all $\left(h^{\prime}, h^{\prime \prime}\right) \in \mathrm{O}\left(V^{\prime}\right)\left(\mathbb{A}_{\mathbb{Q}}\right) \times \mathrm{O}\left(V^{\prime \prime}\right)\left(\mathbb{A}_{\mathbb{Q}}\right)$. Hence we have

$$
I(\mathfrak{c})=2^{-2} \int_{\left[\mathrm{SO}\left(V^{\prime}\right)\right]} \int_{\left[\mathrm{SO}\left(V^{\prime \prime}\right)\right]} \theta\left(h^{\prime} h^{\prime \prime} \phi_{2}(t) ; \mathbf{f}, \varphi\right) \overline{\mathcal{G}\left(h^{\prime} \phi_{2}^{\prime}(t)\right)} d h^{\prime \prime} d h^{\prime} .
$$

Since the isomorphism $K^{1} \stackrel{\cong}{\rightarrow} \mathrm{SO}\left(V^{\prime \prime}\right) \hookrightarrow \mathrm{SO}(V)$ is given by $\alpha \mapsto \phi_{2}(\bar{\alpha})$, we find that

$$
I(\mathfrak{c})=2^{-1} \int_{\left[\mathrm{SO}\left(V^{\prime}\right)\right]} \int_{K^{1} \backslash \mathbb{A}_{K}^{1} / \hat{\mathfrak{o}}_{K}^{1} \mathbb{C}^{1}} \theta\left(h^{\prime} \phi_{2}(\alpha) \phi_{2}(t) ; \mathbf{f}, \varphi\right) \overline{\mathcal{G}\left(h^{\prime} \phi_{2}^{\prime}(t)\right)} d \alpha d h^{\prime} .
$$

We see that the map $\beta \mapsto \beta \bar{\beta}^{-1}$ gives an isomorphism

$$
K^{\times} \backslash \mathbb{A}_{K}^{\times} / \hat{\mathfrak{o}}_{K}^{\times} \mathbb{C}^{\times} \cong K^{1} \backslash \mathbb{A}_{K}^{1} / \hat{\mathfrak{o}}_{K}^{1} \mathbb{C}^{1} .
$$

Since there is a canonical isomorphism $K^{\times} \backslash \mathbb{A}_{K}^{\times} / \hat{\mathfrak{o}}_{K}^{\times} \mathbb{C}^{\times} \cong C l_{K}$, we have

$$
I(\mathfrak{c})=\frac{1}{2 h_{K}} \sum_{i=1}^{h_{K}} \int_{\left[\mathrm{SO}\left(V^{\prime}\right)\right]} \theta\left(h^{\prime} \phi_{2}\left(\beta_{i}{\overline{\beta_{i}}}^{-1} t\right) ; \mathbf{f}, \varphi\right) \overline{\mathcal{G}\left(h^{\prime} \phi_{2}^{\prime}(t)\right)} d h^{\prime},
$$

where $\left\{\beta_{1}, \ldots, \beta_{h_{K}}\right\}$ is a complete system of representatives of $K^{\times} \backslash \mathbb{A}_{K}^{\times} / \hat{\mathfrak{o}}_{K}^{\times} \mathbb{C}^{\times}$. We may take $\beta_{i}$ such that the ideal $\mathfrak{b}_{i}$ defined by $\beta_{i}$ is in $J_{K}^{D}$ for $i=1, \ldots, h_{K}$. We identify $\mathrm{G}\left(\mathrm{SL}_{2} \times \mathrm{SL}_{2}\right)$ as 
a subgroup of $\mathrm{GU}(2,2)$ via the inclusion $\tau$ defined in Sect. 7. If $h^{\prime}=\rho\left(x_{1}, x_{2}\right)$ with $\left(x_{1}, x_{2}\right) \in$ $\mathrm{G}\left(\mathrm{SL}_{2} \times \mathrm{SL}_{2}\right)$, by Corollary 6.9 , we have

$$
\theta\left(h^{\prime} \phi_{2}\left(\beta \bar{\beta}^{-1} t\right) ; \mathbf{f}, \varphi\right)=\left(\prod_{p \mid D} q_{p}^{-1}\right) 2^{2 \kappa+2} a_{f}(D)^{-1} L_{i f t}^{(2)}(f)\left(\tau\left(x_{1}, x_{2}\right) r_{\beta \bar{\beta}^{-1} t}\right),
$$

and

$$
\mathcal{G}\left(h^{\prime} \phi_{2}^{\prime}(t)\right)=\mathbf{g}\left(x_{1}\right) \mathbf{g}\left(x_{2} \cdot d\left(N_{K / \mathbb{Q}}(t)^{-1}\right)\right) .
$$

Moreover we see that the automorphic form $\mathbf{g}\left(x \cdot d\left(N_{K / \mathbb{Q}}(t)^{-1}\right)\right)$ is given by $\left.g\right|_{2 \kappa+2} d(C)=$ $C^{-\kappa-1} g_{C}$. So we have

$$
\begin{aligned}
2^{-1} \int_{\left[\mathrm{SO}\left(V^{\prime}\right)\right]} L_{i f t}^{(2)}(f)\left(\tau\left(x_{1}, x_{2}\right) r_{\beta \bar{\beta}^{-1}}\right) \overline{\mathcal{G}\left(h^{\prime} \phi_{2}^{\prime}(t)\right)} d h^{\prime} \\
=2^{-1} \int_{\left[\mathrm{G}\left(\mathrm{SL}_{2} \times \mathrm{SL}_{2}\right) / \mathrm{GL}_{1}\right]} C^{-\kappa-1}\left[F_{\mathfrak{b}_{\mathfrak{i}} \overline{\mathfrak{b}}_{i}}{ }^{-1} \mathfrak{c} \|_{2 \kappa+2} \tau\left(x_{1}, x_{2}\right)\right](\mathbf{i}) \\
\quad \times \overline{\mathbf{g}\left(x_{1}\right) \mathbf{g}\left(x_{2} \cdot d\left(N_{K / \mathbb{Q}}(t)^{-1}\right)\right)} d\left(x_{1}, x_{2}\right) \\
=(3 / \pi)^{2} C^{-\kappa-1}\left\langle\left. F_{\mathfrak{b}_{\mathfrak{i}} \overline{\mathfrak{b}}_{i}}{ }^{-1}{ }_{\mathfrak{c}}\right|_{\mathfrak{H} \times \mathfrak{H}}, g \times\left(C^{-\kappa-1} g_{C}\right)\right\rangle \\
=\left(2 \xi_{\mathbb{Q}}(2)\right)^{-2} C^{-2 \kappa-2}\left\langle F_{\mathfrak{b}_{\mathfrak{i}} \overline{\mathfrak{b}}_{i}}^{-1} \mathfrak{c} \mid \mathfrak{H} \times \mathfrak{H}, g \times g_{C}\right\rangle .
\end{aligned}
$$

Now we have

$$
\left\langle g_{C}, g_{C}\right\rangle=C^{2(\kappa+1)}\langle g|d(C), g| d(C)\rangle=C^{2(\kappa+1)}\langle g, g\rangle .
$$

Since $\left[\overline{\mathfrak{b}_{i}}\right]=\left[\mathfrak{b}_{i}\right]^{-1}$ in $C l_{K}$, by Lemma 3.1, we have

$$
\begin{aligned}
& I(\mathfrak{c})=\left(\prod_{p \mid D} q_{p}^{-1}\right) 2^{2 \kappa+2} a_{f}(D)^{-1}\left(2 \xi_{\mathbb{Q}}(2)\right)^{-2} \frac{1}{h_{K}} \sum_{[\mathfrak{b}] \in C l_{K}} \frac{\left\langle\left. F_{\mathfrak{b}^{-1} \mathfrak{c}}\right|_{\mathfrak{H} \times \mathfrak{H}}, g \times g_{C}\right\rangle\langle g, g\rangle}{\left\langle g_{C}, g_{C}\right\rangle} \\
& =2^{-1} \xi_{\mathbb{Q}}(2)^{-2}\left(\prod_{p \mid D} q_{p}^{-1}\right) 2^{2 \kappa+1} a_{f}(D)^{-1} \frac{1}{\#\left(C l_{K}^{2}\right)} \sum_{[\mathfrak{a}] \in C l_{K}^{2}} \frac{\left\langle\left. F_{\mathfrak{a} \mathfrak{c}}\right|_{\mathfrak{H} \times \mathfrak{H}, g \times g_{A C}}\right\rangle\langle g, g\rangle}{\left\langle g_{A C}, g_{A C}\right\rangle} .
\end{aligned}
$$

This completes the proof using Lemma 6.7.

Next, we give another expression of $I(\mathfrak{c})$. To do this, we need the following lemmas.

Lemma 9.2. We have

$$
\varphi=\varphi^{\prime} \otimes \varphi^{\prime \prime}
$$

In particular, we have

$$
\begin{aligned}
& \theta\left(g \cdot d\left(\nu \circ \phi_{2}(t)\right), h^{\prime} h^{\prime \prime} \phi_{2}(t) ; \varphi\right) \\
& =\theta\left(g \cdot d\left(\nu \circ \phi_{2}^{\prime}(t)\right), h^{\prime} \phi_{2}^{\prime}(t) ; \varphi^{\prime}\right) \theta\left(g \cdot d\left(\nu \circ \phi_{2}^{\prime \prime}(t)\right), h^{\prime \prime} \phi_{2}^{\prime \prime}(t) ; \varphi^{\prime \prime}\right) .
\end{aligned}
$$

Proof. This is obvious.

Lemma 9.3. The integral

$$
\int_{\left[\mathrm{O}\left(V^{\prime}\right)\right]} \int_{\left[\mathrm{O}\left(V^{\prime \prime}\right)\right]} \int_{\left[\mathrm{SL}_{2}\right]}\left|\theta\left(g \cdot d\left(\nu \circ \phi_{2}(t)\right), h^{\prime} h^{\prime \prime} \phi_{2}(t) ; \varphi\right) \mathbf{f}\left(g \cdot d\left(\nu \circ \phi_{2}(t)\right)\right) \overline{\mathcal{G}\left(h^{\prime} \phi_{2}^{\prime}(t)\right)}\right| d g d h^{\prime \prime} d h^{\prime}
$$

is finite.

Proof. By the above lemma, this integral is equal to

$$
\begin{aligned}
& \int_{\left[\mathrm{O}\left(V^{\prime}\right)\right]} \int_{\left[\mathrm{SL}_{2}\right]}\left|\theta\left(g \cdot d\left(N_{K / \mathbb{Q}}(t)\right), h^{\prime} \phi_{2}^{\prime}(t) ; \varphi^{\prime}\right) \mathbf{f}\left(g \cdot d\left(N_{K / \mathbb{Q}}(t)\right)\right) \overline{\mathcal{G}\left(h^{\prime} \phi_{2}^{\prime}(t)\right)}\right| \\
& \times\left(\int_{\left[\mathrm{O}\left(V^{\prime \prime}\right)\right]}\left|\theta\left(g \cdot d\left(N_{K / \mathbb{Q}}(t)\right), h^{\prime \prime} \phi_{2}^{\prime \prime}(t) ; \varphi^{\prime \prime}\right)\right| d h^{\prime \prime}\right) d g d h^{\prime} .
\end{aligned}
$$


Since $V^{\prime \prime}$ is anisotropic over $\mathbb{Q}$, the space $\left[\mathrm{O}\left(V^{\prime \prime}\right)\right]$ is compact. Hence the inner integral converges and it is slowly increasing function on $\left[\mathrm{SL}_{2}\right]$. Since $\mathbf{f}$ and $\mathcal{G}$ are cusp forms, they are rapidly decreasing. Moreover the function

$$
\left(h^{\prime}, g\right) \mapsto \theta\left(g \cdot d\left(N_{K / \mathbb{Q}}(t)\right), h^{\prime} \phi_{2}^{\prime}(t) ; \varphi^{\prime}\right)
$$

is slowly increasing on $\left[\mathrm{O}\left(V^{\prime}\right)\right] \times\left[\mathrm{SL}_{2}\right]$. Therefore the function

$$
\begin{aligned}
& \left|\theta\left(g \cdot d\left(N_{K / \mathbb{Q}}(t)\right), h^{\prime} \phi_{2}^{\prime}(t) ; \varphi^{\prime}\right) \mathbf{f}\left(g \cdot d\left(N_{K / \mathbb{Q}}(t)\right)\right) \overline{\mathcal{G}\left(h^{\prime} \phi_{2}^{\prime}(t)\right)}\right| \\
& \quad \times\left(\int_{\left[\mathrm{O}\left(V^{\prime \prime}\right)\right]}\left|\theta\left(g \cdot d\left(N_{K / \mathbb{Q}}(t)\right), h^{\prime \prime} \phi_{2}^{\prime \prime}(t) ; \varphi^{\prime \prime}\right)\right| d h^{\prime \prime}\right)
\end{aligned}
$$

is bounded on $\left[\mathrm{O}\left(V^{\prime}\right)\right] \times\left[\mathrm{SL}_{2}\right]$. This completes the proof.

Proposition 9.4. Put $q_{p}=\left(\mathrm{SL}_{2}\left(\mathbb{Z}_{p}\right): K_{0}\left(D ; \mathbb{Z}_{p}\right)\right)$. Then

$$
\begin{aligned}
I(\mathfrak{c})= & 2^{-2} \xi_{\mathbb{Q}}(2)^{-2}\left(\prod_{p \mid D} q_{p}^{-1}\right)(2 \pi)^{-(2 \kappa+1)} a_{f}(D)^{-1}(2 \kappa) !\langle g, g\rangle \\
& \times L(1, \chi)^{-1} \sum_{Q \subset Q_{D}} \chi_{Q}(-C) a_{f_{Q}}(D) L\left(1 / 2, f_{Q} \times g\right) .
\end{aligned}
$$

Proof. By Lemma 7.1, Proposition 8.1 and Lemma 9.3, we have

$$
\begin{aligned}
I(\mathfrak{c}) & =\int_{\left[\mathrm{SL}_{2}\right]}\left(2^{2 \kappa+1} \xi_{\mathbb{Q}}(2)^{-2}\langle g, g\rangle \overline{\mathbf{g}\left(\alpha \cdot d\left(N_{K / \mathbb{Q}}(t)\right)\right)}\right)\left(\frac{1}{2} E(\alpha, 0)\right) \mathbf{f}\left(\alpha \cdot d\left(N_{K / \mathbb{Q}}(t)\right)\right) d \alpha \\
& =2^{-1} 2^{2 \kappa+1} \xi_{\mathbb{Q}}(2)^{-2}\langle g, g\rangle \int_{\left[\mathrm{SL}_{2}\right]} E(\alpha, 0) \mathbf{f}\left(\alpha \cdot d\left(N_{K / \mathbb{Q}}(t)\right)\right) \overline{\mathbf{g}\left(\alpha \cdot d\left(N_{K / \mathbb{Q}}(t)\right)\right)} d \alpha .
\end{aligned}
$$

Here, the Eisenstein series $E(\alpha, s)$ is defined by using the section

$$
\Phi\left(\alpha, s, L\left(\phi_{2}^{\prime \prime}(t) \varphi^{\prime \prime}\right)\right) \text {. }
$$

We put

$$
I(\mathfrak{c}, s)=\int_{\left[\mathrm{SL}_{2}\right]} E(\alpha, s) \mathbf{f}\left(\alpha \cdot d\left(N_{K / \mathbb{Q}}(t)\right)\right) \overline{\mathbf{g}\left(\alpha \cdot d\left(N_{K / \mathbb{Q}}(t)\right)\right)} d \alpha .
$$

Then, we have

$$
I(\mathfrak{c})=\left.2^{-1} 2^{2 \kappa+1} \xi_{\mathbb{Q}}(2)^{-2}\langle g, g\rangle I(\mathfrak{c}, s)\right|_{s=0} .
$$

We formally compute $I(\mathfrak{c}, s)$ for $\operatorname{Re}(s) \gg 0$. Note that $C=N(\mathfrak{c}) \in \mathbb{Q}^{\times} \subset \mathbb{A}_{\mathbb{Q}}^{\times}$, g has the trivial central character and $\mathbf{f}, \mathbf{g}$ are left $\mathrm{GL}_{2}(\mathbb{Q})$-invariant. So we have

$$
\begin{aligned}
I(\mathfrak{c}, s) & =\int_{B(\mathbb{Q}) \backslash \mathrm{SL}_{2}\left(\mathbb{A}_{\mathbb{Q}}\right)} \Phi(\alpha, s) \mathbf{f}\left(\alpha \cdot d\left(N_{K / \mathbb{Q}}(t)\right)\right) \overline{\mathbf{g}\left(\alpha \cdot d\left(N_{K / \mathbb{Q}}(t)\right)\right)} d \alpha \\
& =\int_{B(\mathbb{Q}) \backslash \mathrm{SL}_{2}\left(\mathbb{A}_{\mathbb{Q}}\right)} \Phi(\alpha, s) \mathbf{f}\left(a(C) \alpha d\left(N_{K / \mathbb{Q}}(t)\right)\right) \overline{\mathbf{g}\left(a(C) \alpha d\left(N_{K / \mathbb{Q}}(t)\right)\right)} d \alpha \\
& =\int_{B(\mathbb{Q}) \backslash \mathrm{SL}_{2}\left(\mathbb{A}_{\mathbb{Q}}\right)} \Phi(\alpha, s) \mathbf{f}\left(a(C) \alpha d\left(N_{K / \mathbb{Q}}(t)\right)\right) \sum_{\xi \in \mathbb{Q}^{\times}} \overline{W_{\mathbf{g}}\left(a(\xi C) \alpha d\left(N_{K / \mathbb{Q}}(t)\right)\right)} d \alpha .
\end{aligned}
$$

Note that

$$
\begin{aligned}
\sum_{\xi \in \mathbb{Q}^{\times}} \overline{W_{\mathbf{g}}\left(a(\xi C) \alpha d\left(N_{K / \mathbb{Q}}(t)\right)\right)} d \alpha & =\sum_{\xi \in \mathbb{Q}^{\times} / \mathbb{Q}^{\times 2}} \sum_{\gamma \in \mathbb{Q}_{>0}} \overline{W_{\mathbf{g}}\left(a\left(\xi \gamma^{2} C\right) \alpha d\left(N_{K / \mathbb{Q}}(t)\right)\right)} d \alpha \\
& =\frac{1}{2} \sum_{\xi \in \mathbb{Q}^{\times} / \mathbb{Q}^{\times 2}} \sum_{\gamma \in \mathbb{Q}^{\times}} \overline{W_{\mathbf{g}}\left(a(\xi C) t(\gamma) \alpha d\left(N_{K / \mathbb{Q}}(t)\right)\right)} d \alpha .
\end{aligned}
$$


Hence $I(\mathfrak{c})$ is equal to

$$
\begin{aligned}
& \frac{1}{2} \sum_{\xi \in \mathbb{Q}^{\times} / \mathbb{Q}^{\times 2}} \int_{N(\mathbb{Q}) \backslash \mathrm{SL}_{2}\left(\mathbb{A}_{\mathbb{Q}}\right)} \Phi(\alpha, s) \mathbf{f}\left(a(C) \alpha d\left(N_{K / \mathbb{Q}}(t)\right) \overline{W_{\mathbf{g}}\left(a(\xi C) \alpha d\left(N_{K / \mathbb{Q}}(t)\right)\right)} d \alpha\right. \\
&= \frac{1}{2} \sum_{\xi \in \mathbb{Q}^{\times} / \mathbb{Q}^{\times 2}} \int_{N(\mathbb{Q}) \backslash \mathrm{SL}_{2}\left(\mathbb{A}_{\mathbb{Q}}\right)} \Phi(\alpha, s) \\
& \times \sum_{\xi^{\prime} \in \mathbb{Q}^{\times}} W_{\mathbf{f}}\left(a\left(\xi^{\prime} C\right) \alpha d\left(N_{K / \mathbb{Q}}(t)\right)\right) \overline{W_{\mathbf{g}}\left(a(\xi C) \alpha d\left(N_{K / \mathbb{Q}}(t)\right)\right)} d \alpha \\
&= \frac{1}{2} \sum_{\xi \in \mathbb{Q}^{\times} / \mathbb{Q}^{\times 2}} \sum_{\xi^{\prime} \in \mathbb{Q}^{\times}} \int_{N\left(\mathbb{A}_{\mathbb{Q}}\right) \backslash \mathrm{SL}_{2}\left(\mathbb{A}_{\mathbb{Q}}\right)}\left(\int_{\mathbb{Q} \backslash \mathbb{A}_{\mathbb{Q}}} \psi\left(\xi^{\prime} C x\right) \overline{\psi(\xi C x)} d x\right) \\
&= \frac{1}{2} \sum_{\xi \in \mathbb{Q}^{\times} / \mathbb{Q}^{\times 2}} \int_{N\left(\mathbb{A}_{\mathbb{Q}}\right) \backslash \mathrm{SL}_{2}\left(\mathbb{A}_{\mathbb{Q}}\right)} \Phi(\alpha, s) \\
& \times W_{\mathbf{f}}\left(a(\xi C) \alpha d\left(N_{K / \mathbb{Q}}(t)\right)\right) \overline{W_{\mathbf{g}}\left(a(\xi C) \alpha d\left(N_{K / \mathbb{Q}}(t)\right)\right)} d \alpha \\
&= \frac{1}{2} \sum_{\xi \in \mathbb{Q}^{\times} / \mathbb{Q}^{\times 2}} \prod_{v} I_{v}(\mathfrak{c}, s, \xi)=\frac{1}{2} \sum_{\xi \in \mathbb{Z}} \prod_{v} I_{v}(\mathfrak{c}, s, \xi) . \\
& \text { square free }
\end{aligned}
$$

Here, $\quad \sum_{\xi \in \mathbb{Z}}$ means the sum over all square free integers, and we put

square free

$$
\begin{aligned}
& I_{v}(\mathfrak{c}, s, \xi) \\
& =\int_{N\left(\mathbb{Q}_{v}\right) \backslash \mathrm{SL}_{2}\left(\mathbb{Q}_{v}\right)} \Phi_{v}(\alpha, s) W_{\mathbf{f}, v}\left(a(\xi C) \alpha d\left(N_{K / \mathbb{Q}}(t) v_{v}\right)\right) \overline{W_{\mathbf{g}, v}\left(a(\xi C) \alpha d\left(N_{K / \mathbb{Q}}(t)_{v}\right)\right)} d \alpha .
\end{aligned}
$$

This calculation is justified by the following lemma.

Lemma 9.5. We define $J_{v}\left(\mathfrak{c}, s, \xi, \xi^{\prime}\right)$ by

$$
\int_{N\left(\mathbb{Q}_{v}\right) \backslash \mathrm{SL}_{2}\left(\mathbb{Q}_{v}\right)}\left|\Phi_{v}(\alpha, s) W_{\mathbf{f}, v}\left(a\left(\xi^{\prime} C\right) \alpha d\left(N_{K / \mathbb{Q}}(t)_{v}\right)\right) \overline{W_{\mathbf{g}, v}\left(a(\xi C) \alpha d\left(N_{K / \mathbb{Q}}(t)_{v}\right)\right)}\right| d \alpha .
$$

If $\operatorname{Re}(s)>6$, then

$$
\sum_{\substack{\xi \in \mathbb{Z} \\ \text { square free }}} \sum_{\xi^{\prime} \in \mathbb{Q}^{\times}} \prod_{v} J_{v}\left(\mathfrak{c}, s, \xi, \xi^{\prime}\right)<\infty .
$$

Proof. We need the following lemma which will be proved in the last section.

Lemma 9.6. We put $\sigma=\operatorname{Re}(s)>6$.

(1) For $v=\infty$, we have

$$
I_{\infty}(\mathfrak{c}, s, \xi)= \begin{cases}(4 \pi)^{-(s / 2+2 \kappa+1)}|C \xi|_{\infty}^{-s / 2+1 / 2} \Gamma(s / 2+2 \kappa+1) & \text { if } \xi>0, \\ 0 & \text { if } \xi<0 .\end{cases}
$$

If $\xi, \xi^{\prime}>0$, then

$$
J_{\infty}\left(\mathfrak{c}, s, \xi, \xi^{\prime}\right) \leq(4 \pi)^{-(\sigma / 2+2 \kappa+1)}(C \xi)^{-\sigma / 4+1 / 2}\left(C \xi^{\prime}\right)^{-\sigma / 4} \Gamma(\sigma / 2+2 \kappa+1)
$$

and otherwise, $J_{\infty}\left(\mathfrak{c}, s, \xi, \xi^{\prime}\right)=0$.

(2) For $v=p \nmid C D$, we have

$$
I_{p}(\mathfrak{c}, s, \xi, \gamma)=|\xi|_{p}^{-s / 2+1 / 2} \sum_{n=0}^{\infty}\left|\xi p^{2 n}\right|_{p}^{s / 2+2 \kappa+1} a_{f}\left(\left(\xi p^{2 n}\right)_{p}\right) \overline{a_{g}\left(\left(\xi p^{2 n}\right)_{p}\right)}
$$


and

$$
J_{p}\left(\mathfrak{c}, s, \xi, \xi^{\prime}\right)=|\xi|_{p}^{\kappa+1}\left|\xi^{\prime}\right|_{p}^{\kappa+1 / 2} \sum_{n=0}^{\infty}\left(p^{-n}\right)^{\sigma+4 \kappa+2}\left|a_{f}\left(\left(\xi^{\prime} p^{2 n}\right)_{p}\right) \overline{a_{g}\left(\left(\xi p^{2 n}\right)_{p}\right)}\right| .
$$

(3) For $v=p \mid D$, we have

$$
\begin{aligned}
I_{p}(\mathfrak{c}, s, \xi)= & \frac{1}{\left(\mathrm{SL}_{2}\left(\mathbb{Z}_{p}\right): K_{0}\left(D ; \mathbb{Z}_{p}\right)\right)} a_{f}\left(D_{p}\right)^{-1}|\xi|_{p}^{-s / 2+1 / 2} \sum_{n=0}^{\infty}\left|\xi p^{2 n}\right|_{p}^{s / 2+2 \kappa+1} \\
& \times\left[a_{f}\left(\left(D \xi p^{2 n}\right)_{p}\right)+\underline{\chi}_{p}(-C \xi) \overline{a_{f}\left(\left(D \xi p^{2 n}\right)_{p}\right)}\right] \overline{a_{g}\left(\left(\xi p^{2 n}\right)_{p}\right)}
\end{aligned}
$$

and

$$
\begin{aligned}
J_{p}\left(\mathfrak{c}, s, \xi, \xi^{\prime}\right) \leq & \frac{2\left|a_{F}\left(D_{p}\right)\right|^{-1}}{\left(\mathrm{SL}_{2}\left(\mathbb{Z}_{p}\right): K_{0}(D)\right)}|\xi|_{p}^{\kappa+1}\left|\xi^{\prime}\right|_{p}^{\kappa+1 / 2} \\
& \quad \times \sum_{n=0}^{\infty}\left(p^{-n}\right)^{\sigma+4 \kappa+2}\left|a_{f}\left(\left(D \xi^{\prime} p^{2 n}\right)_{p}\right) \overline{a_{g}\left(\left(\xi p^{2 n}\right)_{p}\right)}\right|
\end{aligned}
$$

(4) For $v=p \mid C$, we have

$$
I_{p}(\mathfrak{c}, s, \xi)=p^{-1 / 2} \frac{p^{s}+p}{1+p}|\xi|_{p}^{-s / 2+1 / 2} \sum_{n=0}^{\infty}\left|\xi p^{2 n}\right|_{p}^{s / 2+2 \kappa+1} a_{f}\left(\left(\xi p^{2 n}\right)_{p}\right) \overline{a_{g}\left(\left(\xi p^{2 n}\right)_{p}\right)}
$$

and

$$
\begin{aligned}
J_{p}\left(\mathfrak{c}, s, \xi, \xi^{\prime}\right)= & p^{-1 / 2} \frac{p^{\sigma}+p}{1+p}|\xi|_{p}^{\kappa+1}\left|\xi^{\prime}\right|_{p}^{\kappa+1 / 2} \\
& \times \sum_{n=0}^{\infty}\left(p^{-n}\right)^{\sigma+4 \kappa+2}\left|a_{f}\left(\left(\xi^{\prime} p^{2 n}\right)_{p}\right) \overline{a_{g}\left(\left(\xi p^{2 n}\right)_{p}\right)}\right| .
\end{aligned}
$$

By this lemma, there is a constant $M_{1}=M_{1}(\sigma)>0$ which does not depend on $\xi, \xi^{\prime}$ and satisfies

$$
\begin{aligned}
& \prod_{v} J_{v}\left(\mathfrak{c}, s, \xi, \xi^{\prime}\right) \\
& \leq M_{1} \xi^{-\sigma / 4-\kappa-1 / 2}\left(\xi^{\prime}\right)^{-\sigma / 4-\kappa-1 / 2} \prod_{p<\infty} \sum_{n=0}^{\infty}\left(p^{-n}\right)^{\sigma+4 \kappa+2}\left|a_{f}\left(\left(D \xi^{\prime} p^{2 n}\right)_{p}\right) \overline{a_{g}\left(\left(\xi p^{2 n}\right)_{p}\right)}\right| \\
& =M_{1} \xi^{-\sigma / 4-\kappa-1 / 2}\left(\xi^{\prime}\right)^{-\sigma / 4-\kappa-1 / 2} \sum_{m=1}^{\infty} m^{-(\sigma+4 \kappa+2)}\left|a_{f}\left(D \xi^{\prime} m^{2}\right) \overline{a_{g}\left(\xi m^{2}\right)}\right|
\end{aligned}
$$

if $\xi, \xi^{\prime}>0$. Otherwise, $\prod_{v} J_{v}\left(\mathfrak{c}, s, \xi, \xi^{\prime}\right)=0$. We put $t=\sigma / 4+\kappa+1 / 2$. Then we have

$$
\begin{aligned}
& \sum_{\substack{\xi \in \mathbb{Z} \\
\text { square free }}} \sum_{\xi^{\prime} \in \mathbb{Q}^{\times}} \prod_{v} J_{v}\left(\mathfrak{c}, s, \xi, \xi^{\prime}\right) \\
\leq & M_{1} \sum_{m=1}^{\infty} \sum_{\substack{\xi \in \mathbb{Z}_{>0} \\
\text { square free }}} \sum_{\xi^{\prime} \in \mathbb{Q}_{>0}^{\times}} \xi^{-t}\left(\xi^{\prime}\right)^{-t} m^{-4 t} \mid a_{f}\left(D \xi^{\prime} m^{2}\right) \overline{a_{g}\left(\xi m^{2}\right) \mid} .
\end{aligned}
$$

Unless $\xi^{\prime} \in\left(D m^{2}\right)^{-1} \mathbb{Z}_{>0}$, we have $\left|a_{f}\left(D \xi^{\prime} m^{2}\right) \overline{a_{g}\left(\xi m^{2}\right)}\right|=0$. So we have

$$
\begin{aligned}
& \sum_{\substack{\xi \in \mathbb{Z} \\
\text { square free }}} \sum_{\xi^{\prime} \in \mathbb{Q}^{\times}} \prod_{v} J_{v}\left(\mathfrak{c}, s, \xi, \xi^{\prime}\right) \\
\leq & M_{1} \sum_{m=1}^{\infty} \sum_{\substack{\xi \in \mathbb{Z}_{>0} \\
\text { square free }}} \sum_{n \in \mathbb{Z}_{>0}} \xi^{-t}\left(\frac{n}{D m^{2}}\right)^{-t} m^{-4 t} \mid a_{f}(n) \overline{a_{g}\left(\xi m^{2}\right)} .
\end{aligned}
$$


It is well-known that

$$
\left|a_{f}(n)\right|=O\left(n^{\kappa+1 / 2}\right), \quad\left|a_{g}(n)\right|=O\left(n^{\kappa+1}\right) .
$$

Hence, there exists a constant $M_{2}>0$ such that

$$
\begin{aligned}
& \sum_{\substack{\xi \in \mathbb{Z} \\
\text { square free }}} \sum_{\xi^{\prime} \in \mathbb{Q}^{x}} \prod_{v} J_{v}\left(\mathfrak{c}, s, \xi, \xi^{\prime}\right) \\
\leq & M_{2} \sum_{m=1}^{\infty} \sum_{\substack{\xi \in \mathbb{Z}_{>0} \\
\text { square free }}} \sum_{n \in \mathbb{Z}_{>0}} \xi^{-t}\left(\frac{n}{D m^{2}}\right)^{-t} m^{-4 t} n^{\kappa+1 / 2}\left(\xi m^{2}\right)^{\kappa+1} \\
\leq & M_{2} D^{t} \sum_{m=1}^{\infty} \sum_{\xi=1}^{\infty} \sum_{n=1}^{\infty} \xi^{-\sigma / 4+1 / 2} m^{-\sigma / 2+1} n^{-\sigma / 4} \\
= & M_{2} D^{t} \zeta(\sigma / 4-1 / 2) \zeta(\sigma / 2-1) \zeta(\sigma / 2) .
\end{aligned}
$$

This is finite if $\sigma>6$.

We return to the proof of Proposition 9.4. By Lemma 9.6, for $\operatorname{Re}(s) \gg 0$, we have

$$
\begin{aligned}
I(\mathfrak{c}, s)= & \frac{1}{2} \sum_{\substack{\xi \in \mathbb{Z} \\
\text { square free }}} \prod_{v} I_{v}(\mathfrak{c}, s, \xi) \\
= & \frac{1}{2}(4 \pi)^{-(s / 2+2 \kappa+1)} C^{-s / 2} \Gamma(s / 2+2 \kappa+1)\left(\prod_{q \mid C} \frac{q^{s}+q}{1+q}\right)\left(\prod_{p \mid D} q_{p}^{-1}\right) \\
& \times a_{f}(D)^{-1} \sum_{\xi \in \mathbb{Z}_{>0}} \sum_{n=1}^{\infty}\left(\xi n^{2}\right)^{-(s / 2+2 \kappa+1)} \alpha_{F_{\mathfrak{c}}}\left(D \xi n^{2}\right) \overline{a_{g}\left(\xi n^{2}\right)} \\
= & \frac{1}{2}(4 \pi)^{-(s / 2+2 \kappa+1)} C^{-s / 2} \Gamma(s / 2+2 \kappa+1)\left(\prod_{q \mid C} \frac{q^{s}+q}{1+q}\right)\left(\prod_{p \mid D} q_{p}^{-1}\right) \\
& \times a_{f}(D)^{-1} \sum_{m=1}^{\infty} m^{-(s / 2+2 \kappa+1)} \alpha_{F_{\mathfrak{c}}}(D m) \overline{a_{g}(m)}
\end{aligned}
$$

Recall that $a_{f \mathfrak{c} *}(D m)=\mathbf{a}_{D}^{\mathfrak{c}}(D m) \alpha_{F_{\mathfrak{c}}}(D m)$. By definition of $\mathbf{a}_{D}^{\mathfrak{c}}$, we have $\mathbf{a}_{D}^{\mathfrak{c}}(D m)=1$. So we have

$$
\alpha_{F_{\mathfrak{c}}}(D m)=a_{f^{\mathfrak{c} *}}(D m)=\sum_{Q \subset Q_{D}} \chi_{Q}(-C) a_{f_{Q}}(D m)=\sum_{Q \subset Q_{D}} \chi_{Q}(-C) a_{f_{Q}}(D) a_{f_{Q}}(m) .
$$

Hence we have

$$
\begin{aligned}
& \sum_{m=1}^{\infty} m^{-(s / 2+2 \kappa+1)} \alpha_{F_{\mathfrak{c}}}(D m) \overline{a_{g}(m)} \\
& =\sum_{Q \subset Q_{D}} \chi_{Q}(-C) a_{f_{Q}}(D) \sum_{m=1}^{\infty} m^{-(s / 2+2 \kappa+1)} a_{f^{\mathfrak{c} *}}(m) \overline{a_{g}(m)} \\
& =\sum_{Q \subset Q_{D}} \chi_{Q}(-C) a_{f_{Q}}(D) \mathcal{D}\left(s / 2+2 \kappa+1, f_{Q}, g\right) \\
& =\sum_{Q \subset Q_{D}} \chi_{Q}(-C) a_{f_{Q}}(D) L(s+1, \chi)^{-1} L\left(s / 2+1 / 2, f_{Q} \times g\right) .
\end{aligned}
$$

This completes the proof of Proposition 9.4 using Lemma 9.6.

By Proposition 9.1 and 9.4, we get the following corollary using Lemma 6.7 and Lemma 9.6. 
Corollary 9.7. For $\mathfrak{c} \in J_{\mathfrak{o}}^{D}$ such that $C=N(\mathfrak{c})$ is a square free integer, we have

$$
\begin{aligned}
& \sum_{Q \subset Q_{D}} \chi_{Q}(-C) a_{f_{Q}}(D) L\left(1 / 2, f_{Q} \times g\right) \\
= & \frac{2 L(1, \chi)(4 \pi)^{2 \kappa+1}}{(2 \kappa) !} \frac{1}{\#\left(C l_{K}^{2}\right)} \sum_{[\mathfrak{a}] \in C l_{K}^{2}} \frac{\left\langle\left. F_{\mathfrak{a c}}\right|_{\mathfrak{H} \times \mathfrak{H}}, g \times g_{A C}\right\rangle}{\left\langle g_{A C}, g_{A C}\right\rangle} .
\end{aligned}
$$

9.2. Genus theory. We recall the genus theory (see e.g., Hecke [12] $\S 48$ ). Let $\mathfrak{G}=C l_{K} / C l_{K}^{2}$. Note that the canonical homomorphism $J_{\mathfrak{o}}^{D} \rightarrow \mathfrak{G}$ is surjective by the Chebotarev density theorem. Let $N: J_{\mathfrak{o}}^{D} \rightarrow \mathbb{Z}_{>0}$ be the ideal norm map. For $Q \subset Q_{D}$, the map $\chi_{Q} \circ N: J_{\mathfrak{o}}^{D} \rightarrow\{ \pm 1\}$ gives a character of $\mathfrak{G}$. Moreover, for $Q, Q^{\prime} \subset Q_{D}$, we see that $\chi_{Q} \circ N=\chi_{Q^{\prime}} \circ N$ if and only if $Q^{\prime}=Q$ or $Q^{\prime}=Q_{D} \backslash Q$. In particular, $\chi_{Q} \circ N$ is the trivial character of $\mathfrak{G}$ if and only if $Q=\emptyset$ or $Q=Q_{D}$. So if we choose $\mathfrak{c}_{1}, \ldots, \mathfrak{c}_{l} \in J_{\mathfrak{o}}^{D}$ such that they give a complete system of $\mathfrak{G}$, then we have

$$
\frac{1}{\left(C l_{K}: C l_{K}^{2}\right)} \sum_{j=1}^{l} \chi_{Q}\left(-N\left(\mathfrak{c}_{j}\right)\right)= \begin{cases}1 & \text { if } Q=\emptyset, \\ -1 & \text { if } Q=Q_{D}, \\ 0 & \text { otherwise }\end{cases}
$$

since $\chi(-1)=-1$. We may assume that $C_{j}=N\left(\mathfrak{c}_{j}\right)$ is a square free integer for each $j=1, \ldots, l$. Considering $\left(C l_{K}: C l_{K}^{2}\right)^{-1} \sum_{j=1}^{l} I\left(\mathfrak{c}_{j}\right)$, by Corollary 9.7 and Lemma 3.1, we have

$$
\begin{aligned}
& a_{f_{\emptyset}}(D) L\left(1 / 2, f_{\emptyset} \times g\right)-a_{f_{Q_{D}}}(D) L\left(1 / 2, f_{Q_{D}} \times g\right) \\
& =\frac{2 L(1, \chi)(4 \pi)^{2 \kappa+1}}{(2 \kappa) !} \frac{1}{\#\left(C l_{K}^{2}\right)} \frac{1}{\left(C l_{K}: C l_{K}^{2}\right)} \sum_{j=1}^{l} \sum_{[\mathfrak{a}] \in C l_{K}^{2}} \frac{\left\langle\left. F_{\mathfrak{a c}}\right|_{\mathfrak{H} \times \mathfrak{H}}, g \times g_{A C_{j}}\right\rangle}{\left\langle g_{A C_{j}}, g_{A C_{j}}\right\rangle} \\
& =\frac{2 L(1, \chi)(4 \pi)^{2 \kappa+1}}{(2 \kappa) !} \frac{1}{h_{K}} \sum_{[\mathfrak{c}] \in C l_{K}} \frac{\left\langle\left. F_{\mathfrak{c}}\right|_{\mathfrak{H} \times \mathfrak{H}}, g \times g_{C}\right\rangle}{\left\langle g_{C}, g_{C}\right\rangle} .
\end{aligned}
$$

Now we find that

$$
\begin{aligned}
& a_{f_{\emptyset}}(D) L\left(1 / 2, f_{\emptyset} \times g\right)=a_{f}(D) L(1 / 2, f \times g), \\
& a_{f_{Q_{D}}}(D) L\left(1 / 2, f_{Q_{D}} \times g\right)=\overline{a_{f}(D) L(1 / 2, f \times g)} .
\end{aligned}
$$

Since $a_{f}(D) L(1 / 2, f \times g) \in \sqrt{-1} \mathbb{R}$, we have

$$
a_{f_{\emptyset}}(D) L\left(1 / 2, f_{\emptyset} \times g\right)-a_{f_{Q_{D}}}(D) L\left(1 / 2, f_{Q_{D}} \times g\right)=2 a_{f}(D) L(1 / 2, f \times g) .
$$

This completes the proof of Theorem 3.2 using Lemma 6.7 and Lemma 9.6.

\section{The LOCAL INTEGRALS}

In this section, we prove Lemma 6.7 and Lemma 9.6. We show these lemmas only when $v=\infty, v=p=2$ or $v=p \mid C_{i}$ (or $\left.v=p \mid C\right)$. The other cases are shown similarly.

10.1. Preliminaries. Through this section, we use the notation

$$
B=\left(\begin{array}{cc}
b_{1} & b_{2}+\sqrt{-D} b_{3} \\
b_{2}-\sqrt{-D} b_{3} & b_{4}
\end{array}\right)
$$

and we put $\xi=\operatorname{det}(B), \beta=\beta(B), m_{i}=\operatorname{ord}_{p}\left(b_{i}\right)$ and $l=\operatorname{ord}_{p}(\xi)$ when we prove Lemma 6.7 as in Sect. 6.4. We fix a place $v$ of $\mathbb{Q}$. Let $\psi=\psi_{v}$ be the standard character of $\mathbb{Q}_{v}$.

Since the anisotropic kernel of $V$ is isomorphic to the one of $V^{\prime \prime}$ over $\mathbb{Q}$, we find that $\gamma_{V\left(\mathbb{Q}_{v}\right)}=$ $\gamma_{V^{\prime \prime}\left(\mathbb{Q}_{v}\right)}$ and $\chi_{V\left(\mathbb{Q}_{v}\right)}=\chi_{V^{\prime \prime}\left(\mathbb{Q}_{v}\right)}=\underline{\chi}_{v}$. Moreover, we have $\gamma_{V^{\prime}\left(\mathbb{Q}_{v}\right)}=1$ and $\chi_{V^{\prime}\left(\mathbb{Q}_{v}\right)}=1$. We simply denote $\gamma_{V\left(\mathbb{Q}_{v}\right)}$ by $\gamma_{V}$ and $\chi_{V\left(\mathbb{Q}_{v}\right)}$ by $\chi_{V}$. It is easily seen that $\operatorname{vol}\left(V\left(\mathbb{Z}_{p}\right)\right)=\operatorname{vol}\left(V^{\prime \prime}\left(\mathbb{Z}_{p}\right)\right)=$ $|4 D|_{p}^{1 / 2}$ and $\operatorname{vol}\left(V^{\prime}\left(\mathbb{Z}_{p}\right)\right)=1$ if $v=p<\infty$, and

$$
\gamma_{V}=\gamma_{V^{\prime \prime}}=(2,-1)_{\mathbb{Q}_{v}} \gamma_{\mathbb{Q}_{v}}(D, \psi) \gamma_{\mathbb{Q}_{v}}(\psi)^{2} \text {. }
$$


Let $\varphi^{\prime} \in \mathcal{S}\left(V^{\prime}\left(\mathbb{Q}_{v}\right)\right)$ and $\varphi^{\prime \prime} \in \mathcal{S}\left(V^{\prime \prime}\left(\mathbb{Q}_{v}\right)\right)$ and we put $\varphi=\varphi^{\prime} \otimes \varphi^{\prime \prime} \in \mathcal{S}\left(V\left(\mathbb{Q}_{v}\right)\right)$. Then we have

$$
\omega_{V}(g, 1) \varphi=\left(\omega_{V^{\prime}}(g, 1) \varphi^{\prime}\right) \otimes\left(\omega_{V^{\prime \prime}}(g, 1) \varphi^{\prime \prime}\right) .
$$

for $g \in \mathrm{SL}_{2}\left(\mathbb{Q}_{v}\right)$. If $v=p<\infty$, for open compact subsets $A, B \subset \mathbb{Q}_{p}$, we denote the characteristic function of of $A e_{3}+B e_{4} \subset V^{\prime \prime}\left(\mathbb{Q}_{p}\right)$ by $\varphi^{\prime \prime}(A, B) \in \mathcal{S}\left(V^{\prime \prime}\left(\mathbb{Q}_{p}\right)\right)$. Let $\varphi^{\prime} \in \mathcal{S}\left(V^{\prime}\left(\mathbb{Q}_{p}\right)\right)$ be the characteristic function of $V^{\prime}\left(\mathbb{Z}_{p}\right)$. Then we find that $\varphi^{\prime}$ is $\mathrm{SL}_{2}\left(\mathbb{Z}_{p}\right)$-invariant. We put $\varphi(A, B)=\varphi^{\prime} \otimes \varphi^{\prime \prime}(A, B) \in \mathcal{S}\left(V\left(\mathbb{Q}_{p}\right)\right)$.

Let $v=p<\infty$. We put $q_{p}=\left(\mathrm{SL}_{2}\left(\mathbb{Z}_{p}\right): K_{0}\left(D ; \mathbb{Z}_{p}\right)\right)$. It is well-known that for $N \in p \mathbb{Z}_{p}$, a complete system of representatives of $\mathrm{SL}_{2}\left(\mathbb{Z}_{p}\right) / K_{0}\left(N ; \mathbb{Z}_{p}\right)$ is given by

$$
\mathcal{A}\left(N ; \mathbb{Z}_{p}\right)=\left\{\left(\begin{array}{ll}
1 & 0 \\
c & 1
\end{array}\right) \mid c \in \mathbb{Z}_{p} / N \mathbb{Z}_{p}\right\} \cup\left\{\left(\begin{array}{cc}
a & -1 \\
1 & 0
\end{array}\right) \mid a \in p \mathbb{Z}_{p} / N \mathbb{Z}_{p}\right\} .
$$

Note that

$$
\left(\begin{array}{ll}
1 & 0 \\
c & 1
\end{array}\right)=-w \cdot n(-c) \cdot w, \quad\left(\begin{array}{cc}
a & -1 \\
1 & 0
\end{array}\right)=n(a) \cdot w
$$

If $c \neq 0$, we have $-w \cdot n(-c) \cdot w=t\left(c^{-1}\right) \cdot n(c) \cdot w \cdot n\left(c^{-1}\right)$.

10.2. The archimedean case. We will prove Lemma 6.7 (1) and Lemma 9.6 (1). Let $v=\infty$. We define the functions $f$ and $q^{\prime}$ on $V^{\prime}(\mathbb{R})$ and $q^{\prime \prime}$ on $V^{\prime \prime}(\mathbb{R})$ by

$$
\begin{aligned}
f\left(u^{\prime}\right) & =-\sqrt{-1} u_{1}+u_{2}-u_{5}+\sqrt{-1} u_{6} \quad \text { and } \\
q^{\prime}\left(u^{\prime}\right) & =u_{1}^{2}+u_{2}^{2}+u_{5}^{2}+u_{6}^{2}, \quad q^{\prime \prime}\left(u^{\prime \prime}\right)=2 u_{3}^{2}+2 D u_{4}^{2}=2 Q^{\prime \prime}\left[u^{\prime \prime}\right]
\end{aligned}
$$

for $u^{\prime}=u_{1} e_{1}+u_{2} e_{2}+u_{5} e_{5}+u_{6} e_{6} \in V^{\prime}(\mathbb{R})$ and $u^{\prime \prime}=u_{3} e_{3}+u_{4} e_{4} \in V^{\prime \prime}(\mathbb{R})$. We regard $f$ as a function on $V(\mathbb{R})$ by $f\left(u^{\prime}+u^{\prime \prime}\right)=f\left(u^{\prime}\right)$ for $u^{\prime} \in V^{\prime}(\mathbb{R})$ and $u^{\prime \prime} \in V^{\prime \prime}(\mathbb{R})$. We put $q(u)=q^{\prime}\left(u^{\prime}\right)+q^{\prime \prime}\left(u^{\prime \prime}\right)$ for $u=u^{\prime}+u^{\prime \prime} \in V(\mathbb{R})$ with $u^{\prime} \in V^{\prime}(\mathbb{R})$ and $u^{\prime \prime} \in V^{\prime \prime}(\mathbb{R})$. We consider

$$
\varphi^{\prime}\left(u^{\prime}\right)=f\left(u^{\prime}\right)^{2 \kappa+2} e^{-\pi q^{\prime}\left(u^{\prime}\right)} \in \mathcal{S}\left(V^{\prime}(\mathbb{R})\right), \quad \varphi^{\prime \prime}\left(u^{\prime \prime}\right)=e^{-\pi q^{\prime \prime}\left(u^{\prime \prime}\right)} \in \mathcal{S}\left(V^{\prime \prime}(\mathbb{R})\right),
$$

and we put $\varphi=\varphi^{\prime} \otimes \varphi^{\prime \prime} \in \mathcal{S}(V(\mathbb{R}))$.

Proposition 10.1. For $k_{\theta} \in \mathrm{SO}(2)$, we have

$$
\omega_{V^{\prime}(\mathbb{R})}\left(k_{\theta}, 1\right) \varphi^{\prime}\left(u^{\prime}\right)=e^{-\sqrt{-1}(2 \kappa+2) \theta} \varphi^{\prime}\left(u^{\prime}\right), \quad \omega_{V^{\prime \prime}(\mathbb{R})}\left(k_{\theta}, 1\right) \varphi^{\prime \prime}\left(u^{\prime \prime}\right)=e^{\sqrt{-1} \theta} \varphi^{\prime \prime}\left(u^{\prime \prime}\right) .
$$

So we have

$$
\omega_{V(\mathbb{R})}\left(k_{\theta}, \phi_{1}\left(k^{\prime}\right) \phi_{2}(t)\right) \varphi(u)=e^{-\sqrt{-1}(2 \kappa+1) \theta} \operatorname{det}(\alpha+\sqrt{-1} \beta)^{2 \kappa+2} \varphi(u)
$$

for

$$
k^{\prime}=\left(\begin{array}{cc}
\alpha & \beta \\
-\beta & \alpha
\end{array}\right) \in \mathbb{K}_{0}
$$

and $t \in \mathbb{C}^{1}$. In particular, the functions

$$
\begin{aligned}
& \mathrm{SL}_{2}(\mathbb{R}) \ni g \longmapsto \hat{\omega}_{V(\mathbb{R})}\left(g, \phi_{1}\left(\left(z \mathbf{1}_{4}\right) n(X) m(A) k^{\prime}\right) \phi_{2}(t)\right) \hat{\varphi}(-\beta ; 0,1) W_{\mathbf{f}, \infty}(a(\xi) g), \\
& \mathrm{SL}_{2}(\mathbb{R}) \ni g \longmapsto \Phi\left(g, s, \varphi^{\prime \prime}\right) W_{\mathbf{f}, \infty}\left(a\left(\xi^{\prime}\right) g\right) \overline{W_{\mathbf{g}, \infty}(a(\xi) g)}
\end{aligned}
$$

are $\mathrm{SO}(2)$-invariant for all $z \in \mathbb{C}^{\times}, X \in \operatorname{Her}_{2}(\mathbb{R}), A \in \mathrm{GL}_{2}(\mathbb{C})$ with $\operatorname{det}(A) \in \mathbb{R}^{\times}, k^{\prime} \in \mathbb{K}_{0}$, $t \in \mathbb{C}^{\times}$with $|t|=1, \xi, \xi^{\prime} \in \mathbb{Q}^{\times}$and $s \in \mathbb{C}$.

Proof. Note that $k^{\prime-1}={ }^{t} \overline{k^{\prime}}$ and $\operatorname{det}\left(k^{\prime}\right)=\operatorname{det}(\alpha+\sqrt{-1} \beta) \operatorname{det}(\alpha-\sqrt{-1} \beta)=1$. By a simple calculation, we have

$$
f\left(\phi_{2}(t)^{-1} \phi_{1}(k)^{-1} u\right)=\operatorname{det}(\alpha+\sqrt{-1} \beta) f(u), \quad q\left(\phi_{2}(t)^{-1} \phi_{1}(k)^{-1} u\right)=q(u)
$$

for $u \in V(\mathbb{R})$. These equations give the actions of $k^{\prime}$ and $t$. 
To study the action of $\mathrm{SO}(2)$ on $\mathcal{S}\left(V^{\prime}(\mathbb{R})\right)$ and on $\mathcal{S}\left(V^{\prime \prime}(\mathbb{R})\right)$, consider the action of the Lie algebra $\operatorname{Lie}\left(\mathrm{SL}_{2}(\mathbb{R})\right)$. Note that $k_{\theta}=\exp \left(\theta\left(X_{1}-X_{2}\right)\right)$ with

$$
X_{1}=\left(\begin{array}{ll}
0 & 1 \\
0 & 0
\end{array}\right), X_{2}=\left(\begin{array}{ll}
0 & 0 \\
1 & 0
\end{array}\right) \in \operatorname{Lie}\left(\mathrm{SL}_{2}(\mathbb{R})\right) .
$$

By the classical Fourier analysis, we can calculate the actions of $X_{1}$ and $X_{2}$.

Now, we start to prove Lemma 6.7 (1). The proof of Lemma 6.7 (1) is similar to that of Lemma 7.6 of [14]. By Proposition 10.1, we may assume that $x_{\infty}=n(X) m(A)$. By [14] Lemma 7.4 , we find that $\hat{\varphi}\left(x ; y_{1}, y_{2}\right)$ is equal to

$$
\begin{aligned}
(2 \sqrt{\pi})^{-(2 \kappa+2)} & H_{2 \kappa+2}\left(\sqrt{\pi}\left(x_{1}-x_{4}+\sqrt{-1} y_{1}+y_{4}\right)\right) \\
& \times \exp \left(-\pi\left(\left(x_{1}-x_{4}+y_{2}\right)^{2}-2 y_{2}\left(x_{1}-x_{4}\right)+2 Q_{1}[x]+y_{1}^{2}\right)\right)
\end{aligned}
$$

for $x={ }^{t}\left(x_{1}, x_{2}, x_{3}, x_{4}\right) \in V_{1}(\mathbb{R})$ and $y_{1}, y_{2} \in \mathbb{R}$. Here,

$$
H_{n}(x)=(-1)^{n} e^{x^{2}} \frac{d^{n}}{d x^{n}}\left(e^{-x^{2}}\right)
$$

is the Hermite polynomial. For $A \in \mathrm{GL}_{2}(\mathbb{C})$ with $\operatorname{det}(A) \in \mathbb{R}^{\times}$, we have

$$
\phi_{1}(m(A))=\left(\begin{array}{ccc}
\operatorname{det}(A) & 0 & 0 \\
0 & h_{1} & 0 \\
0 & 0 & \operatorname{det}(A)^{-1}
\end{array}\right)
$$

with some $h_{1} \in \operatorname{GSO}(3,1)(\mathbb{R})$. By a simple calculation, we have

$$
-\left(h_{1}^{-1} \beta\right)_{1}+\left(h_{1}^{-1} \beta\right)_{4}=\operatorname{det}(A)^{-1} \operatorname{Tr}\left(B A^{t} \bar{A}\right)=\operatorname{det}(A)^{-1} \operatorname{Tr}(B Y) .
$$

Let $v=v(X)$ as in Sect. 6.4. Then we have $\phi_{1}(n(X))=\ell(v),(v, \beta)_{V_{1}}=\operatorname{Tr}(B X)$ and $Q_{1}\left[h_{1}^{-1} \beta\right]=$ $Q_{1}[\beta]=-\operatorname{det}(B)=-\xi$. So we have

$$
\begin{aligned}
\hat{\omega} & (t(x), \phi(n(X) m(A))) \hat{\varphi}(-\beta ; 0,1) \\
= & \chi_{V}(x)|x|_{\infty}^{3} \int_{\mathbb{R}} \varphi\left(\begin{array}{c}
x \operatorname{det}(A)^{-1} z-x \operatorname{det}(A)^{-1}(v, \beta) \\
-h_{1}^{-1} \beta x \\
0
\end{array}\right) e^{2 \pi \sqrt{-1} z} d z \\
= & \chi_{V}(x)|x|_{\infty}^{2}|\operatorname{det}(A)|_{\infty} e^{2 \pi \sqrt{-1} \operatorname{Tr}(B X)} \hat{\varphi}\left(-h_{1}^{-1} \beta x ; 0, \operatorname{det}(A) x^{-1}\right) \\
= & \chi_{V}(x)|x|_{\infty}^{2}|\operatorname{det}(A)|_{\infty} e^{2 \pi \sqrt{-1} \operatorname{Tr}(B X)} H_{2 \kappa+2}\left(\sqrt{\pi}\left[\operatorname{det}(A)^{-1} x \operatorname{Tr}(B Y)+\operatorname{det}(A) x^{-1}\right]\right) \\
& \times(4 \pi)^{-\kappa-1} \exp \left(-\pi\left[\operatorname{det}(A)^{-1} x \operatorname{Tr}(B Y)+\operatorname{det}(A) x^{-1}\right]^{2}\right) \exp \left(2 \pi \operatorname{Tr}(B Y)+2 \pi x^{2} \xi\right) .
\end{aligned}
$$

By the formula of $W_{\mathbf{f}, \infty}$ in Sect. 4.3, we have $\mathcal{W}_{B, \infty}(n(X) m(A))=0$ if $\xi<0$. If $\xi>0$, then $\mathcal{W}_{B, \infty}(n(X) m(A))$ is equal to

$$
\begin{aligned}
& \int_{\mathbb{R}^{\times}} \hat{\omega}\left(t(x), \phi_{1}(n(X) m(A))\right) \hat{\varphi}(-\beta ; 0,1) W_{\mathbf{f}, \infty}(a(\xi) t(x))|x|_{\infty}^{-2} d^{\times} x \\
& =2 \int_{0}^{\infty} \hat{\omega}\left(t(x), \phi_{1}(n(X) m(A))\right) \hat{\varphi}(-\beta ; 0,1) W_{\mathbf{f}, \infty}(a(\xi) t(x)) x^{-3} d x \\
& =2|\operatorname{det}(A)|^{2 \kappa+2} e^{2 \pi \sqrt{-1} \operatorname{Tr}(B(X-Y \sqrt{-1}))}(4 \pi)^{-\kappa-1} \xi^{\kappa+(1 / 2)} \\
& \quad \times \int_{0}^{\infty} x^{2 \kappa} e^{-\pi\left[x \operatorname{Tr}(B Y)+x^{-1}\right]^{2}} H_{2 \kappa+2}\left(\sqrt{\pi}\left[x \operatorname{Tr}(B Y)+x^{-1}\right]\right) d x .
\end{aligned}
$$

By [14] Lemma 7.5, the last integral is equal to

$$
\begin{cases}2^{2(2 \kappa+2)-1} \pi^{\kappa+1} e^{4 \pi \sqrt{-1} \operatorname{Tr}(B Y \sqrt{-1})} & \text { if } B>0, \\ 0 & \text { if } B<0 .\end{cases}
$$

Since $Y=A^{t} \bar{A}$, we have $\operatorname{det}(Y)=|\operatorname{det}(A)|^{2}$. This completes the proof of Lemma 6.7 (1). 
Lemma 9.6 (1) follows from the equation $\Phi\left(t(x), s, \varphi^{\prime \prime}\right)=\chi_{V^{\prime \prime}(\mathbb{R})}(x)|x|_{\infty}^{s+1}$, the formulas of $W_{\mathbf{f}, \infty}$ and $W_{\mathbf{g}, \infty}$ in Sect. 4.3, and the well-known formula

$$
\int_{\mathbb{R}^{\times}}|x|^{s} e^{-a x^{2}} d^{\times} x=a^{-s / 2} \Gamma\left(\frac{s}{2}\right)
$$

for $a>0$.

10.3. The unramified 2-adic case. We will prove Lemma 6.7 (2) and Lemma 9.6 (2) for $p=2$. Let $v=2 \nmid D$. So we find that $D \equiv 3 \bmod 4$.

In this case, we have $(2,-1)_{\mathbb{Q}_{2}}=1, \gamma_{\mathbb{Q}_{2}}(D, \psi)=\sqrt{-1}$ and $\gamma_{\mathbb{Q}_{2}}(\psi)=\zeta_{8}^{-1}$. Hence we have $\gamma_{V}=\gamma_{V^{\prime \prime}}=1$.

Let $\varphi^{\prime \prime}=\varphi^{\prime \prime}\left(\mathbb{Z}_{2}, \mathbb{Z}_{2}\right)+\varphi^{\prime \prime}\left(2^{-1} \mathbb{Z}_{2}^{\times}, 2^{-1} \mathbb{Z}_{2}^{\times}\right)$and $\varphi_{0}^{\prime \prime}=\varphi^{\prime \prime}\left(\mathbb{Z}_{2}, \mathbb{Z}_{2}\right) \in \mathcal{S}\left(V^{\prime \prime}\left(\mathbb{Q}_{2}\right)\right)$. We put $\varphi=\varphi^{\prime} \otimes \varphi^{\prime \prime}$ and $\varphi_{0}=\varphi^{\prime} \otimes \varphi_{0}^{\prime \prime}$.

Lemma 10.2. We have

$$
\varphi^{\prime \prime}(u)=2 \int_{\mathrm{SL}_{2}\left(\mathbb{Z}_{2}\right)} \omega(k, 1) \varphi_{0}^{\prime \prime}(u) d k \quad \text { and so } \quad \varphi(u)=2 \int_{\mathrm{SL}_{2}\left(\mathbb{Z}_{2}\right)} \omega(k, 1) \varphi_{0}(u) d k .
$$

In particular, $\varphi$ and $\varphi^{\prime \prime}$ is $\mathrm{SL}_{2}\left(\mathbb{Z}_{2}\right)$-invariant. Moreover $\varphi$ is also $\phi_{1}\left(\operatorname{GSU}(2,2)\left(\mathbb{Z}_{2}\right)\right) \phi_{2}\left(\left(\mathfrak{o} \otimes_{\mathbb{Z}}\right.\right.$ $\left.\mathbb{Z}_{2}\right)^{\times}$)-invariant.

Proof. It is easy that $\varphi_{0}^{\prime \prime}$ is $K_{0}\left(4 ; \mathbb{Z}_{2}\right)$-invariant. For $a, c \in \mathbb{Z}_{2}$, we have

$$
\begin{aligned}
\omega(-w \cdot n(-c) \cdot w, 1) \varphi_{0}^{\prime \prime}(u) & =\omega(-w, 1)\left[\left(\omega(w, 1) \varphi_{0}^{\prime \prime}\right](u)\right) \psi\left(-c Q^{\prime \prime}[u]\right), \\
\omega(n(a) w, 1) \varphi_{0}^{\prime \prime} & =\left[\omega(w, 1) \varphi_{0}^{\prime \prime}\right](u) \psi\left(a Q^{\prime \prime}[u]\right) .
\end{aligned}
$$

Now we find that

$$
\sum_{c \in \mathbb{Z}_{2} / 4 \mathbb{Z}_{2}} \psi\left(-c Q^{\prime \prime}[u]\right)= \begin{cases}4 & \text { if } Q^{\prime \prime}[u] \in \mathbb{Z}_{2} \\ 0 & \text { otherwise }\end{cases}
$$

Hence, we have

$$
\sum_{c \in \mathbb{Z}_{2} / 4 \mathbb{Z}_{2}}\left(\omega(w, 1) \varphi_{0}^{\prime \prime}(u)\right) \psi\left(-c Q^{\prime \prime}[u]\right)=4 \operatorname{vol}\left(V^{\prime \prime}\left(\mathbb{Z}_{2}\right)\right) \varphi^{\prime \prime}(u) .
$$

Similarly, we have

$$
\sum_{a \in 2 \mathbb{Z}_{2} / 4 \mathbb{Z}_{2}}\left(\omega(w, 1) \varphi_{0}^{\prime \prime}(u)\right) \psi\left(a Q^{\prime \prime}[u]\right)=2 \operatorname{vol}\left(V^{\prime \prime}\left(\mathbb{Z}_{2}\right)\right) \varphi^{\prime \prime}(u) .
$$

On the other hand, $\omega(w, 1) \varphi^{\prime \prime}$ is equal to

$$
\begin{aligned}
& \omega(w, 1)\left[\varphi^{\prime \prime}\left(2^{-1} \mathbb{Z}_{2}, 2^{-1} \mathbb{Z}_{2}\right)-\varphi^{\prime \prime}\left(2^{-1} \mathbb{Z}_{2}, \mathbb{Z}_{2}\right)-\varphi^{\prime \prime}\left(\mathbb{Z}_{2}, 2^{-1} \mathbb{Z}_{2}\right)+2 \varphi^{\prime \prime}\left(\mathbb{Z}_{2}, \mathbb{Z}_{2}\right)\right] \\
& =\operatorname{vol}\left(V^{\prime \prime}\left(\mathbb{Z}_{2}\right)\right)\left[4 \varphi^{\prime \prime}\left(\mathbb{Z}_{2}, \mathbb{Z}_{2}\right)-2 \varphi^{\prime \prime}\left(\mathbb{Z}_{2}, 2^{-1} \mathbb{Z}_{2}\right)-2 \varphi^{\prime \prime}\left(2^{-1} \mathbb{Z}_{2}, \mathbb{Z}_{2}\right)+2 \varphi^{\prime \prime}\left(2^{-1} \mathbb{Z}_{2}, 2^{-1} \mathbb{Z}_{2}\right)\right] .
\end{aligned}
$$

Since $\operatorname{vol}\left(V^{\prime \prime}\left(\mathbb{Z}_{2}\right)\right)=\left|\operatorname{det}\left(Q^{\prime \prime}\right)\right|^{1 / 2}=2^{-1}$, we have $\omega(w, 1) \varphi^{\prime \prime}=\varphi^{\prime \prime}$. Therefore we have

$$
\begin{aligned}
& \int_{\mathrm{SL}_{2}\left(\mathbb{Z}_{2}\right)} \omega(k, 1) \varphi_{0}^{\prime \prime}(u) d k \\
& =\left(\mathrm{SL}_{2}\left(\mathbb{Z}_{2}\right): K_{0}\left(4 ; \mathbb{Z}_{2}\right)\right)^{-1}\left[4 \operatorname{vol}\left(V^{\prime \prime}\left(\mathbb{Z}_{2}\right)\right) \varphi^{\prime \prime}(u)+2 \operatorname{vol}\left(V^{\prime \prime}\left(\mathbb{Z}_{2}\right)\right) \varphi^{\prime \prime}(u)\right]=2^{-1} \varphi^{\prime \prime}(u) .
\end{aligned}
$$

The equation for $\varphi$ implies that $\varphi$ is $\mathrm{SL}_{2}\left(\mathbb{Z}_{2}\right) \times \phi_{1}\left(\mathrm{GSU}(2,2)\left(\mathbb{Z}_{2}\right)\right)$-invariant. By Lemma 8.2, we find that $\varphi$ is $\phi_{2}\left(\left(\mathfrak{o} \otimes_{\mathbb{Z}} \mathbb{Z}_{p}\right)^{\times}\right)$-invariant. 
Now, we start to prove Lemma 6.7 (2) for $p=2$. By Lemma 6.1 and Lemma 10.2, we find that $\mathcal{W}_{B, 2}$ is right $\mathrm{GU}(2,2)\left(\mathbb{Z}_{2}\right)$-invariant. Moreover, we have

$$
\begin{aligned}
\mathcal{W}_{B, 2}(1) & =\int_{\mathbb{Q}_{2}^{\times}} \hat{\omega}(t(x), 1) \hat{\varphi}(-\beta ; 0,1) W_{\mathbf{f}, 2}(a(\xi) t(x))|x|_{2}^{-2} d^{\times} x \\
& =\int_{\mathbb{Q}_{2}^{\times}}\left[\chi_{V}(x)|x|_{2}^{2} \hat{\varphi}\left(-\beta x ; 0, x^{-1}\right)\right]\left[\underline{\chi}_{2}\left(x^{-1}\right) W_{\mathbf{f}, 2}\left(a\left(\xi x^{2}\right)\right)\right]|x|_{2}^{-2} d^{\times} x \\
& =\sum_{n \in \mathbb{Z}} \hat{\varphi}\left(-\beta 2^{-n} ; 0,2^{n}\right)\left|\xi\left(2^{-n}\right)^{2}\right|_{2}^{\kappa+1 / 2} a_{f}\left(\left(\xi\left(2^{-n}\right)^{2}\right)_{2}\right) .
\end{aligned}
$$

Note that $\varphi=\varphi\left(2^{-1} \mathbb{Z}_{2}, 2^{-1} \mathbb{Z}_{2}\right)-\varphi\left(2^{-1} \mathbb{Z}_{2}^{\times}, \mathbb{Z}_{2}\right)-\varphi\left(\mathbb{Z}_{2}, 2^{-1} \mathbb{Z}_{2}^{\times}\right)$.

Lemma 10.3. We have

$$
\sum_{n \in \mathbb{Z}} \varphi\left(2^{-1} \mathbb{Z}_{2}^{\times}, \mathbb{Z}_{2}\right)^{\wedge}\left(-\beta 2^{-n} ; 0,2^{n}\right)\left|\xi 2^{-2 n}\right|_{2}^{\kappa+1 / 2} a_{f}\left(\left(\xi 2^{-2 n}\right)_{2}\right)=0,
$$

and

$$
\sum_{n \in \mathbb{Z}} \varphi\left(\mathbb{Z}_{2}, 2^{-1} \mathbb{Z}_{2}^{\times}\right)^{\wedge}\left(-\beta 2^{-n} ; 0,2^{n}\right)\left|\xi 2^{-2 n}\right|_{2}^{\kappa+1 / 2} a_{f}\left(\left(\xi 2^{-2 n}\right)_{2}\right)=0 .
$$

Proof. Since $\beta={ }^{t}\left(-b_{4},-b_{2},-b_{3}, b_{1}\right)$, we have $\varphi\left(2^{-1} \mathbb{Z}_{2}^{\times}, \mathbb{Z}_{2}\right)^{\wedge}\left(-\beta 2^{-n} ; 0,2^{n}\right)=0$ unless

$$
n \geq 0, \quad m_{4}-n \geq 0, \quad m_{2}-n=-1, m_{3}-n \geq 0, \quad \text { and } \quad m_{1}-n \geq 0 .
$$

In this case, we have $n=m_{2}+1 \leq \min \left(m_{1}, m_{3}, m_{4}\right)$. So we find that

$$
l=\operatorname{ord}_{2}(\xi)=\operatorname{ord}_{2}\left(b_{1} b_{4}-b_{2}^{2}-D b_{3}^{2}\right)=2 m_{2}<2 n .
$$

Therefore we have $a_{f}\left(\left(\xi 2^{-2 n}\right)_{2}\right)=0$. The second assertion is proved similarly.

Let $m_{0}=\min \left(m_{1}, m_{2}+1, m_{3}+1, m_{4}\right)$. Then by this lemma, we have

$$
\begin{aligned}
\mathcal{W}_{B, 2}(1) & =\sum_{n \in \mathbb{Z}} \varphi\left(2^{-1} \mathbb{Z}_{2}, 2^{-1} \mathbb{Z}_{2}\right)^{\kappa}\left(-\beta 2^{-n} ; 0,2^{n}\right)\left|\xi 2^{-2 n}\right|_{2}^{\kappa+1 / 2} a_{f}\left(\left(\xi 2^{-2 n}\right)_{2}\right) \\
& =|\xi|_{2}^{\kappa+1 / 2} \sum_{n=0}^{m_{0}}\left(2^{n}\right)^{2 \kappa+1} a_{f}\left(\left(\xi 2^{-2 n}\right)_{2}\right) .
\end{aligned}
$$

This completes the proof of Lemma 6.7 (2) for $p=2$.

Next, we start to prove Lemma 9.6 (2) (for any $p$ ). Then we have

$$
\begin{aligned}
J_{p}\left(\mathfrak{c}, s, \xi, \xi^{\prime}\right)= & \int_{\mathbb{Q}_{p}^{\times}}\left|\Phi\left(t(x), s, \varphi^{\prime \prime}\right) W_{\mathbf{f}, p}\left(a\left(\xi^{\prime} C\right) t(x)\right) \overline{W_{\mathbf{g}, p}(a(\xi C) t(x))}\right||x|_{p}^{-2} d^{\times} x \\
= & \int_{\mathbb{Q}_{p}^{\times}} \mid\left[\chi_{V^{\prime \prime}\left(\mathbb{Q}_{p}\right)}(x)|x|_{p}^{s+1}\right]\left[\underline{\chi}_{p}\left(x^{-1}\right)\left|\xi^{\prime} C x^{2}\right|_{p}^{\kappa+1 / 2} a_{f}\left(\left(\xi^{\prime} C x^{2}\right)_{p}\right)\right] \\
& \times\left.\left[\overline{\left|\xi C x^{2}\right|_{p}^{\kappa+1} a_{g}\left(\left(\xi C x^{2}\right)_{p}\right)}\right]|| x\right|_{p} ^{-2} d^{\times} x \\
= & |\xi|_{p}^{\kappa+1}\left|\xi^{\prime}\right|_{p}^{\kappa+1 / 2} \int_{\mathbb{Q}_{p}^{\times}}|x|_{p}^{\sigma+4 \kappa+2}\left|a_{f}\left(\left(\xi^{\prime} x^{2}\right)_{p}\right) \overline{a_{g}\left(\left(\xi x^{2}\right)_{p}\right)}\right| d^{\times} x .
\end{aligned}
$$

Since $\operatorname{ord}_{p}(\xi)=0$ or 1 , this is equal to

$$
|\xi|_{p}^{\kappa+1}\left|\xi^{\prime}\right|_{p}^{\kappa+1 / 2} \sum_{n=0}^{\infty}\left(p^{-n}\right)^{\sigma+4 \kappa+2}\left|a_{f}\left(\left(\xi^{\prime} p^{2 n}\right)_{p}\right) \overline{a_{g}\left(\left(\xi p^{2 n}\right)_{p}\right)}\right| .
$$

This is the desired formula for $J_{p}\left(\mathfrak{c}, s, \xi, \xi^{\prime}\right)$. The formula for $I_{p}(\mathfrak{c}, s, \xi)$ can be proved similarly. These complete the proof of Lemma 9.6 (2) (for any $p$ ). 
10.4. The ramified 2-adic case. Let $v=2 \mid D$. We will prove Lemma 6.7 (3) and Lemma 9.6 (3) for this case. We put $d=\operatorname{ord}_{2}(D) \in\{2,3\}$ and $D^{\prime}=D / 2^{d}$. Write $D^{\prime}=\delta u_{0}$ with $\delta \in\{ \pm 1\}$ and $u_{0} \in 1+4 \mathbb{Z}_{2}$. Note that if $d=2$, then $\delta=1$. In this case, we have $(2,-1)_{\mathbb{Q}_{2}}=1$, $\gamma_{\mathbb{Q}_{2}}(\psi)=\zeta_{8}^{-1}$. If $d=2$, then $\gamma_{\mathbb{Q}_{2}}(D, \psi)=\gamma_{\mathbb{Q}_{2}}\left(D^{\prime}, \psi\right)=1$. If $d=3$, then

$$
\underline{\chi}_{2}(2)=\left\{\begin{array}{ll}
1 & \text { if } D^{\prime} \equiv \pm 1 \bmod 8, \\
-1 & \text { if } D^{\prime} \equiv \pm 3 \bmod 8,
\end{array} \gamma_{\mathbb{Q}_{2}}(D, \psi)= \begin{cases}1 & \text { if } D^{\prime} \equiv 1 \bmod 8, \\
-\sqrt{-1} & \text { if } D^{\prime} \equiv 3 \bmod 8, \\
-1 & \text { if } D^{\prime} \equiv 5 \bmod 8, \\
\sqrt{-1} & \text { if } D^{\prime} \equiv 7 \bmod 8 .\end{cases}\right.
$$

Hence we have $\gamma_{V} \varepsilon=\gamma_{V^{\prime \prime}} \varepsilon=\underline{\chi}_{2}\left(2^{d}\right)$.

Let $\varphi^{\prime \prime}=\varphi^{\prime \prime}\left(\mathbb{Z}_{2}, 2^{-1} \mathbb{Z}_{2}\right), \varphi_{0}^{\prime \prime}=\varphi^{\prime \prime}\left(\mathbb{Z}_{2}, \mathbb{Z}_{2}\right), \varphi_{1}^{\prime \prime}=\varphi^{\prime \prime}\left(2^{-1} \mathbb{Z}_{2},(2 D)^{-1} \mathbb{Z}_{2}\right) \in \mathcal{S}\left(V^{\prime \prime}\left(\mathbb{Q}_{2}\right)\right)$. We put $\varphi=\varphi^{\prime} \otimes \varphi^{\prime \prime}$ and $\varphi_{i}=\varphi^{\prime} \otimes \varphi_{i}^{\prime \prime} \in \mathcal{S}\left(V\left(\mathbb{Q}_{2}\right)\right)$ for $i=0,1$. Clearly, $\varphi_{0}$ is $\phi_{1}\left(\operatorname{GSU}(2,2)\left(\mathbb{Z}_{2}\right)\right)$ invariant. We find that

$$
\omega(w, 1) \varphi_{0}^{\prime \prime}=\gamma_{V^{\prime \prime}}^{-1} \operatorname{vol}\left(V^{\prime \prime}\left(\mathbb{Z}_{2}\right)\right) \varphi_{1}^{\prime \prime} \quad \text { and so } \quad \omega(w, 1) \varphi_{0}=\gamma_{V}^{-1} \operatorname{vol}\left(V\left(\mathbb{Z}_{2}\right)\right) \varphi_{1} .
$$

For

we have

$$
k=\left(\begin{array}{ll}
a & b \\
c & d
\end{array}\right)=w^{-1} \cdot n(-c / a) \cdot w \cdot t(a) \cdot n(b / a) \in K_{0}\left(4 D ; \mathbb{Z}_{p}\right)
$$

$$
\omega(k, 1) \varphi_{0}^{\prime \prime}=\chi_{V^{\prime \prime}}(a) \varphi_{0}^{\prime \prime} \quad \text { and so } \quad \omega(k, 1) \varphi_{0}=\chi_{V}(a) \varphi_{0} .
$$

A complete system of representatives of $K_{0}\left(D ; \mathbb{Z}_{2}\right) / K_{0}\left(4 D ; \mathbb{Z}_{2}\right)$ is given by

$$
\left\{\left(\begin{array}{ll}
1 & 0 \\
c & 1
\end{array}\right)=-w \cdot n(-c) \cdot w \mid c \in D \mathbb{Z}_{2} / 4 D \mathbb{Z}_{2}\right\} .
$$

Lemma 10.4. We have

$$
\sum_{c \in D \mathbb{Z}_{2} / 4 D \mathbb{Z}_{2}} \omega(-w \cdot n(-c) \cdot w, 1) \varphi_{0}^{\prime \prime}=2 \varphi^{\prime \prime} \quad \text { and so } \sum_{c \in D \mathbb{Z}_{2} / 4 D \mathbb{Z}_{2}} \omega(-w \cdot n(-c) \cdot w, 1) \varphi_{0}=2 \varphi \text {. }
$$

In particular, $K_{0}\left(D ; \mathbb{Z}_{2}\right)$ acts on $\varphi^{\prime \prime}($ resp. $\varphi)$ by the scalar multiplication of the character

$$
\left(\begin{array}{ll}
a & b \\
c & d
\end{array}\right)=w^{-1} \cdot n(-c / a) \cdot w \cdot t(a) \cdot n(b / a) \mapsto \chi_{V^{\prime \prime}}(a) \quad\left(\text { resp. } \chi_{V}(a)\right) .
$$

Moreover, we find that $\varphi$ is $\phi_{1}\left(\operatorname{GSU}(2,2)\left(\mathbb{Z}_{2}\right)\right) \phi_{2}\left(\left(\mathfrak{o} \otimes_{\mathbb{Z}} \mathbb{Z}_{2}\right)^{\times}\right)$-invariant.

Proof. We have

$$
\omega(n(-c) \cdot w, 1) \varphi^{\prime \prime}(u)=\gamma_{V^{\prime \prime}}^{-1} \operatorname{vol}\left(V\left(\mathbb{Z}_{2}\right)\right) \varphi_{1}^{\prime \prime}(u) \psi\left(-c Q^{\prime \prime}[u]\right)
$$

Note that

We find that

$$
\sum_{c \in D \mathbb{Z}_{2} / 4 D \mathbb{Z}_{2}} \psi\left(-c Q^{\prime \prime}[u]\right)= \begin{cases}4 & \text { if } Q^{\prime \prime}[u] \in D^{-1} \mathbb{Z}_{2}, \\ 0 & \text { if } Q^{\prime \prime}[u] \notin D^{-1} \mathbb{Z}_{2} .\end{cases}
$$

$$
\left\{u \in V^{\prime \prime}\left(\mathbb{Q}_{p}\right) \mid \varphi_{1}^{\prime \prime}(u) \neq 0, Q^{\prime \prime}[u] \in D^{-1} \mathbb{Z}_{2}\right\}=2^{-1} \mathbb{Z}_{2} e_{3}+D^{-1} \mathbb{Z}_{2} e_{4} .
$$

Hence, we have

$$
\sum_{c \in D \mathbb{Z}_{2} / 4 D \mathbb{Z}_{2}} \omega(n(-c) \cdot w, 1) \varphi_{0}^{\prime \prime}=4 \gamma_{V^{\prime \prime}}^{-1} \operatorname{vol}\left(V\left(\mathbb{Z}_{2}\right)\right) \varphi^{\prime \prime}\left(2^{-1} \mathbb{Z}_{2}, D^{-1} \mathbb{Z}_{2}\right) .
$$

Since

$$
\begin{aligned}
\omega(w, 1) \varphi^{\prime \prime}\left(2^{-1} \mathbb{Z}_{2}, D^{-1} \mathbb{Z}_{2}\right) & =\gamma_{V^{\prime \prime}}^{-1} \operatorname{vol}\left(2^{-1} \mathbb{Z}_{2} e_{3}+D^{-1} \mathbb{Z}_{2} e_{4}\right) \varphi^{\prime \prime} \\
& =2^{1+d} \gamma_{V^{\prime \prime}}^{-1} \operatorname{vol}\left(V\left(\mathbb{Z}_{2}\right)\right) \varphi^{\prime \prime}
\end{aligned}
$$


we have

$$
\sum_{c \in D \mathbb{Z}_{2} / 4 D \mathbb{Z}_{2}} \omega(-w \cdot n(-c) \cdot w, 1) \varphi_{0}^{\prime \prime}(u)=2^{3+d} \operatorname{vol}\left(V\left(\mathbb{Z}_{2}\right)\right)^{2} \varphi^{\prime \prime}(-u)=2 \varphi^{\prime \prime}(u) .
$$

Let $k=w^{-1} \cdot n(-c / a) \cdot w \cdot t(a) \cdot n(b / a) \in K_{0}\left(D ; \mathbb{Z}_{2}\right)$ and $c_{1}, c_{2} \in D \mathbb{Z}_{2}$. Then

$$
\left(\begin{array}{cc}
1 & 0 \\
c_{1} & 1
\end{array}\right)^{-1} k\left(\begin{array}{cc}
1 & 0 \\
c_{2} & 1
\end{array}\right)=\left(\begin{array}{cc}
a+b c_{2} & * \\
* & *
\end{array}\right) \in K_{0}\left(D ; \mathbb{Z}_{2}\right)
$$

and $\chi_{V^{\prime \prime}}\left(a+b c_{2}\right)=\chi_{V^{\prime \prime}}(a)$. Therefore we have

$$
\omega(k, 1) \varphi^{\prime \prime}=\chi_{V^{\prime \prime}}(a) \varphi^{\prime \prime} .
$$

The last assertion follows from Lemma 8.2.

By this lemma, we find that the functions

$$
\begin{aligned}
& \mathrm{SL}_{2}\left(\mathbb{Q}_{2}\right) \ni g \longmapsto \hat{\omega}(g, 1) \hat{\varphi}(-\beta ; 0,1) W_{\mathbf{f}, 2}(a(\xi) g), \\
& \mathrm{SL}_{2}\left(\mathbb{Q}_{2}\right) \ni g \longmapsto \Phi\left(g, s, \varphi^{\prime \prime}\right) W_{\mathbf{f}, 2}\left(a\left(\xi^{\prime} C\right) g\right) \overline{W_{\mathbf{g}, 2}(a(\xi C) g)}
\end{aligned}
$$

are right $K_{0}\left(D ; \mathbb{Z}_{2}\right)$-invariant. Hence we have

$$
\begin{gathered}
\mathcal{W}_{B, 2}(1)=\left(\mathrm{SL}_{2}\left(\mathbb{Z}_{2}\right): K_{0}\left(D ; \mathbb{Z}_{2}\right)\right)^{-1} \sum_{k \in \mathrm{SL}_{2}\left(\mathbb{Z}_{2}\right) / K_{0}\left(D ; \mathbb{Z}_{2}\right)} \Omega_{B, 2}(k), \\
I_{2}(\mathfrak{c}, s, \xi)=\left(\mathrm{SL}_{2}\left(\mathbb{Z}_{2}\right): K_{0}\left(D ; \mathbb{Z}_{2}\right)\right)^{-1} \sum_{k \in \mathrm{SL}_{2}\left(\mathbb{Z}_{2}\right) / K_{0}\left(D ; \mathbb{Z}_{2}\right)} \Lambda_{B, 2}(k),
\end{gathered}
$$

where

$$
\begin{aligned}
& \Omega_{B, 2}(k)=\int_{\mathbb{Q}_{2}^{\times}} \hat{\omega}(t(x) k, 1) \hat{\varphi}(-\beta ; 0,1) W_{\mathbf{f}, 2}(a(\xi) t(x) k)|x|_{2}^{-2} d^{\times} x, \\
& \Lambda_{B, 2}(k)=\int_{\mathbb{Q}_{2}^{\times}} \Phi\left(t(x) k, s, \varphi^{\prime \prime}\right) W_{\mathbf{f}, 2}(a(\xi C) t(x) k) \overline{W_{\mathbf{g}, 2}(a(\xi C) t(x) k)}|x|_{2}^{-2} d^{\times} x .
\end{aligned}
$$

A complete system of representatives of $\mathrm{SL}_{2}\left(\mathbb{Z}_{2}\right) / K_{0}\left(D ; \mathbb{Z}_{2}\right)$ is given by

$$
\mathcal{A}\left(D ; \mathbb{Z}_{2}\right)=\left\{-w \cdot n(-c) \cdot w, n(a) \cdot w \mid c \in \mathbb{Z}_{2} / D \mathbb{Z}_{2}, a \in 2 \mathbb{Z}_{2} / D \mathbb{Z}_{2}\right\} .
$$

Lemma 10.5. Let $x \in \mathbb{Q}_{2}^{\times}$. We put $n=\operatorname{ord}_{2}(x)$ and $l=\operatorname{ord}_{2}(\xi)$.

(1) For $k=-w \cdot n(-c) \cdot w$ with $c \in \mathbb{Z}_{2}^{\times}$, we have

$\omega(t(x) k, 1) \varphi^{\prime \prime}(u)=2^{-d / 2} \gamma_{V^{\prime \prime}}^{-1} \chi_{V^{\prime \prime}}\left(x c^{-1}\right)|x|_{2} \varphi^{\prime \prime}\left(2^{-1} \mathbb{Z}_{2}, D^{-1} \mathbb{Z}_{2}\right)(u x) \psi\left(c^{-1} Q^{\prime \prime}[u x]\right)$

so that

$$
\omega(t(x) k, 1) \varphi(u)=2^{-d / 2} \gamma_{V}^{-1} \chi_{V}\left(x c^{-1}\right)|x|_{2}^{3} \varphi\left(2^{-1} \mathbb{Z}_{2}, D^{-1} \mathbb{Z}_{2}\right)(u x) \psi\left(c^{-1} Q[u x]\right),
$$

and $W_{\mathbf{f}, 2}(a(\xi) t(x) k)$ is equal to

$$
2^{-d / 2} \varepsilon^{-1} \psi\left(\xi x^{2} c^{-1}\right) \underline{\chi}_{2}\left(x^{-1} c\right) \underline{\chi}_{2}\left(-2^{d} \xi\right) a_{f}\left(D_{2}\right)^{-1}\left|\xi x^{2}\right|_{2}^{\kappa+1 / 2} \overline{a_{f}\left(\left(D \xi x^{2}\right)_{2}\right)} .
$$

(2) For $k=n(a) \cdot w$ with $a \in 2 \mathbb{Z}_{2}$, we have

$$
\omega(t(x) k, 1) \varphi^{\prime \prime}(u)=2^{-d / 2} \gamma_{V^{\prime \prime}}^{-1} \chi_{V^{\prime \prime}}(x)|x|_{2} \varphi^{\prime \prime}\left(2^{-1} \mathbb{Z}_{2}, D^{-1} \mathbb{Z}_{2}\right)(u x) \psi\left(a Q^{\prime \prime}[u x]\right)
$$

so that

$$
\omega(t(x) k, 1) \varphi(u)=2^{-d / 2} \gamma_{V}^{-1} \chi_{V}(x)|x|_{2}^{3} \varphi\left(2^{-1} \mathbb{Z}_{2}, D^{-1} \mathbb{Z}_{2}\right)(u x) \psi(a Q[u x]),
$$

and $W_{\mathbf{f}, 2}(a(\xi) t(x) k)$ is equal to

$$
2^{-d / 2} \varepsilon^{-1} \psi\left(a \xi x^{2}\right) \underline{\chi}_{2}\left(x^{-1}\right) \underline{\chi}\left(-2^{d} \xi\right) a_{f}\left(D_{2}\right)^{-1}\left|\xi x^{2}\right|_{2}^{\kappa+1 / 2} \overline{a_{f}\left(\left(D \xi x^{2}\right)_{2}\right)} .
$$


(3) For $k=-w \cdot n\left(-2^{d-1}\right) \cdot w=k_{1}$, we have

$$
\omega(t(x) k, 1) \varphi^{\prime \prime}(u)=\chi_{V^{\prime \prime}}(x)|x|_{2} \times \begin{cases}\varphi^{\prime \prime}\left(2^{-1} \mathbb{Z}_{2}^{\times}, 2^{-2} \mathbb{Z}_{2}^{\times}\right)(u x), & \text { if } d=2, \\ \varphi^{\prime \prime}\left(\mathbb{Z}_{2}, 2^{-2} \mathbb{Z}_{2}^{\times}\right)(u x), & \text { if } d=3\end{cases}
$$

so that

$$
\omega(t(x) k, 1) \varphi(u)=\chi_{V}(x)|x|_{2}^{3} \times \begin{cases}\varphi\left(2^{-1} \mathbb{Z}_{2}^{\times}, 2^{-2} \mathbb{Z}_{2}^{\times}\right)(u x), & \text { if } d=2, \\ \varphi\left(\mathbb{Z}_{2}, 2^{-2} \mathbb{Z}_{2}^{\times}\right)(u x), & \text { if } d=3\end{cases}
$$

and

$$
W_{\mathbf{f}, 2}(a(\xi) t(x) k)= \begin{cases}\underline{\chi}_{2}\left(x^{-1}\right)\left|\xi x^{2}\right|_{2}^{\kappa+1 / 2} a_{f}(2)^{-1} & \text { if } l+2 n=-1, \\ 0 & \text { otherwise. }\end{cases}
$$

(4) Assume that $d=3$ and $k=-w \cdot n( \pm 2) \cdot w$. Then $\omega(t(x) k, 1) \varphi^{\prime \prime}(u)$ is equal to $2^{-1} \chi_{V^{\prime \prime}}(x)|x|_{2}$

$$
\times\left[\varphi^{\prime \prime}\left(2^{-1} \mathbb{Z}_{2}^{\times}, 2^{-2} \mathbb{Z}_{2}\right) \pm \delta \sqrt{-1}\left(\varphi^{\prime \prime}\left(2^{-1} \mathbb{Z}_{2}^{\times}, 2^{-2} \mathbb{Z}_{2}^{\times}\right)-\varphi^{\prime \prime}\left(2^{-1} \mathbb{Z}_{2}^{\times}, 2^{-1} \mathbb{Z}_{2}\right)\right)\right](u x)
$$

so that $\omega(t(x) k, 1) \varphi(u)$ is equal to

$$
\begin{aligned}
& 2^{-1} \chi_{V}(x)|x|_{2}^{3} \\
& \times\left[\varphi\left(2^{-1} \mathbb{Z}_{2}^{\times}, 2^{-2} \mathbb{Z}_{2}\right) \pm \delta \sqrt{-1}\left(\varphi\left(2^{-1} \mathbb{Z}_{2}^{\times}, 2^{-2} \mathbb{Z}_{2}^{\times}\right)-\varphi\left(2^{-1} \mathbb{Z}_{2}^{\times}, 2^{-1} \mathbb{Z}_{2}\right)\right)\right](u x),
\end{aligned}
$$

and $W_{\mathbf{f}, 2}(a(\xi) t(x) k)$ is equal to

$$
\begin{cases}\underline{\chi}_{2}\left(x^{-1}\right) 2^{-1 / 2} \underline{\chi}_{2}(\mp \eta) \varepsilon \zeta_{8}^{ \pm \eta}\left|\xi x^{2}\right|_{2}^{\kappa+1 / 2} a_{f}(2)^{-2} & \text { if } l+2 n=-2, \\ 0 & \text { otherwise. }\end{cases}
$$

Proof. (1) Let $k=-w \cdot n(-c) \cdot w=t\left(c^{-1}\right) \cdot n(c) \cdot w \cdot n\left(c^{-1}\right)$. Then we have

$$
\begin{aligned}
& \omega(t(x) k, 1) \varphi^{\prime \prime}(u)=\omega\left(t\left(x c^{-1}\right) \cdot n(c) \cdot w, 1\right) \varphi^{\prime \prime}(u) \\
& =\gamma_{V^{\prime \prime}}^{-1} \operatorname{vol}\left(\mathbb{Z}_{2} e_{3}+2^{-1} \mathbb{Z}_{2} e_{4}\right) \omega\left(t\left(x c^{-1}\right) \cdot n(c), 1\right) \varphi^{\prime \prime}\left(2^{-1} \mathbb{Z}_{2}, D^{-1} \mathbb{Z}_{2}\right)(u) \\
& =2^{-d / 2} \gamma_{V^{\prime \prime}}^{-1} \chi_{V^{\prime \prime}}\left(x c^{-1}\right)\left|x c^{-1}\right|_{2} \varphi^{\prime \prime}\left(2^{-1} \mathbb{Z}_{2}, D^{-1} \mathbb{Z}_{2}\right)\left(u x c^{-1}\right) \psi\left(c Q^{\prime \prime}\left[u x c^{-1}\right]\right) \\
& =2^{-d / 2} \gamma_{V^{\prime \prime}}^{-1} \chi_{V^{\prime \prime}}\left(x c^{-1}\right)|x|_{2} \varphi^{\prime \prime}\left(2^{-1} \mathbb{Z}_{2}, 2^{-2} \mathbb{Z}_{2}\right)(u x) \psi\left(c^{-1} Q^{\prime \prime}[u x]\right) .
\end{aligned}
$$

On the other hand, since $a(\xi) \cdot t\left(x c^{-1}\right) \cdot n(c)=n\left(\xi x^{2} c^{-1}\right) a(\xi) t\left(x c^{-1}\right)$, we have

$$
\begin{aligned}
& W_{\mathbf{f}, 2}(a(\xi) t(x) k)=\psi\left(\xi x^{2} c^{-1}\right) \underline{\chi}_{2}\left(x^{-1} c\right) W_{\mathbf{f}, 2}\left(a\left(\xi x^{2} c^{-2}\right) w\right) \\
& =\psi\left(\xi x^{2} c^{-1}\right) \underline{\chi}_{2}\left(x^{-1} c\right) \underline{\chi}_{2}\left(-D_{2} \xi x^{2}\right) \varepsilon^{-1} 2^{-d / 2} a_{f}\left(D_{2}\right)^{-1}\left|\xi x^{2}\right|_{2}^{\kappa+1 / 2} \overline{a_{f}\left(\left(D \xi x^{2}\right)_{2}\right)} .
\end{aligned}
$$

(2) Let $k=n(a) \cdot w$ with $a \in 2 \mathbb{Z}_{2}$. Then we have

$$
\omega(t(x) k, 1) \varphi^{\prime \prime}(u)=\gamma_{V^{\prime \prime}}^{-1} 2^{-d / 2} \chi_{V^{\prime \prime}}(x)|x|_{2} \varphi^{\prime \prime}\left(2^{-1} \mathbb{Z}_{2}, D^{-1} \mathbb{Z}_{2}\right)(u x) \psi\left(a Q^{\prime \prime}[u x]\right) .
$$

On the other hand, since $a(\xi) t(x) n(a)=n\left(a \xi x^{2}\right) a(\xi) t(x)$, we have

$$
\begin{aligned}
& W_{\mathbf{f}, 2}(a(\xi) t(x) k) \\
& =\psi\left(a \xi x^{2}\right) \underline{\chi}_{2}\left(x^{-1}\right) \underline{\chi}\left(-D_{2} \xi x^{2}\right) \varepsilon^{-1} 2^{-d / 2} a_{f}\left(D_{2}\right)^{-1}\left|\xi x^{2}\right|_{2}^{\kappa+1 / 2} \overline{a_{f}\left(\left(D \xi x^{2}\right)_{2}\right)} .
\end{aligned}
$$

(3) We assume that $d=3$. The proof for $d=2$ is similar. Let $k=-w \cdot n(-4) \cdot w=k_{1}$. The formula for $W_{\mathbf{f}, 2}\left(a(\xi) t(x) k_{1}\right)$ follows from Lemma 4.2 immediately. On the other hand, we have

$$
\omega(n(-4) \cdot w, 1) \varphi^{\prime \prime}(u)=\gamma_{V^{\prime \prime}}^{-1} \operatorname{vol}\left(\mathbb{Z}_{2} e_{3}+2^{-1} \mathbb{Z}_{2} e_{4}\right) \varphi^{\prime \prime}\left(2^{-1} \mathbb{Z}_{2}, 2^{-3} \mathbb{Z}_{2}\right)(u) \psi\left(-4 Q^{\prime \prime}[u]\right) .
$$

Now we find that

$$
\psi\left(-4 Q^{\prime \prime}\left[u_{3} e_{3}+u_{4} e_{4}\right]\right)= \begin{cases}1 & \text { if } u_{3} \in 2^{-1} \mathbb{Z}_{2}, u_{4} \in 2^{-2} \mathbb{Z}_{2}, \\ -1 & \text { if } u_{3} \in 2^{-1} \mathbb{Z}_{2}, u_{4} \in 2^{-3} \mathbb{Z}_{2}^{\times} .\end{cases}
$$


Hence we find that $\omega(n(-4) \cdot w, 1) \varphi^{\prime \prime}(u)$ is equal to

$$
2^{-3 / 2} \gamma_{V^{\prime \prime}}^{-1}\left[\varphi^{\prime \prime}\left(2^{-1} \mathbb{Z}_{2} \cdot 2^{-2} \mathbb{Z}_{2}\right)-\varphi^{\prime \prime}\left(2^{-1} \mathbb{Z}_{2}, 2^{-3} \mathbb{Z}_{2}^{\times}\right)\right](u) .
$$

Note that

$$
\begin{aligned}
\omega(w, 1) \varphi^{\prime \prime}\left(2^{-1} \mathbb{Z}_{2}, 2^{-2} \mathbb{Z}_{2}\right)(u) & =2^{1 / 2} \gamma_{V^{\prime \prime}}^{-1}\left[\varphi^{\prime \prime}\left(\mathbb{Z}_{2}, 2^{-1} \mathbb{Z}_{2}\right)+\varphi^{\prime \prime}\left(\mathbb{Z}_{2}, 2^{-2} \mathbb{Z}_{2}^{\times}\right)\right](u), \\
\omega(w, 1) \varphi^{\prime \prime}\left(2^{-1} \mathbb{Z}_{2}, 2^{-3} \mathbb{Z}_{2}^{\times}\right)(u) & =2^{1 / 2} \gamma_{V^{\prime \prime}}^{-1}\left[\varphi^{\prime \prime}\left(\mathbb{Z}_{2}, 2^{-1} \mathbb{Z}_{2}\right)-\varphi^{\prime \prime}\left(\mathbb{Z}_{2}, 2^{-2} \mathbb{Z}_{2}^{\times}\right)\right](u) .
\end{aligned}
$$

So we have

$$
\omega(w \cdot n(-4) \cdot w, 1) \varphi^{\prime \prime}=\gamma_{V^{\prime \prime}}^{-2} \varphi^{\prime \prime}\left(\mathbb{Z}_{2}, 2^{-2} \mathbb{Z}_{2}^{\times}\right) .
$$

Since $\gamma_{V^{\prime \prime}}^{2}=\underline{\chi}_{2}(-1)$, we have

$$
\omega\left(t(x) k_{1}, 1\right) \varphi^{\prime \prime}(u)=\chi_{V^{\prime \prime}}(x)|x|_{2} \varphi^{\prime \prime}\left(\mathbb{Z}_{2}, 2^{-2} \mathbb{Z}_{2}^{\times}\right)(u x) .
$$

(4) Assume that $d=3$. Let $k=-w \cdot n( \pm 2) \cdot w=a(\mp 1) \cdot k_{2} \cdot a(\mp 1)$. We write $\xi=2^{l} \eta$ and $x=2^{n} u$ with $\eta, u \in \mathbb{Z}_{2}^{\times}$. Then $W_{\mathbf{f}, 2}(a(\xi) t(x) k)$ is equal to

$$
\begin{cases}\underline{\chi}_{2}\left(x^{-1}\right) 2^{-1 / 2} \underline{\chi}_{2}\left(\mp \eta u^{2}\right) \varepsilon \zeta_{8}^{ \pm \eta u^{2}}\left|\mp \xi x^{2}\right|_{2}^{\kappa+1 / 2} a_{f}(2)^{-2} & \text { if } l+2 n=-2, \\ 0 & \text { otherwise. }\end{cases}
$$

Since $u^{2} \equiv 1 \bmod 8$ for $u \in \mathbb{Z}_{2}^{\times}$, we get the desired formula for $W_{\mathbf{f}, 2}(a(\xi) t(x) k)$. On the other hand, we have

$$
\omega(n( \pm 2) \cdot w, 1) \varphi^{\prime \prime}(u)=\gamma_{V^{\prime \prime}}^{-1} \operatorname{vol}\left(\mathbb{Z}_{2} e_{3}+2^{-1} \mathbb{Z}_{2} e_{4}\right) \varphi^{\prime \prime}\left(2^{-1} \mathbb{Z}_{2}, 2^{-3} \mathbb{Z}_{2}\right)(u) \psi\left( \pm 2 Q^{\prime \prime}[u]\right) .
$$

Since $\pm 2 Q^{\prime \prime}\left[u_{3} e_{3}+u_{4} e_{4}\right]=\left( \pm 2\left(u_{3}^{2}+8 \delta u_{0} u_{4}^{2}\right)\right)$, we have

$$
\pm 2 Q^{\prime \prime}\left[u_{3} e_{3}+u_{4} e_{4}\right] \equiv\left\{\begin{array}{lll}
0 & \bmod \mathbb{Z}_{2} & \text { if } u_{3} \in \mathbb{Z}_{2}, u_{4} \in 2^{-2} \mathbb{Z}_{2}, \\
\pm \delta \cdot 4^{-1} & \bmod \mathbb{Z}_{2} & \text { if } u_{3} \in \mathbb{Z}_{2}, u_{4} \in 2^{-3} \mathbb{Z}_{2}^{\times}, \\
\pm 2^{-1} & \bmod \mathbb{Z}_{2} & \text { if } u_{3} \in 2^{-1} \mathbb{Z}_{2}^{\times}, u_{4} \in 2^{-2} \mathbb{Z}_{2}, \\
\pm 2^{-1} \pm \delta \cdot 4^{-1} & \bmod \mathbb{Z}_{2} & \text { if } u_{3} \in 2^{-1} \mathbb{Z}_{2}^{\times}, u_{4} \in 2^{-3} \mathbb{Z}_{2}^{\times}
\end{array}\right.
$$

Since $\psi(1 / 4)=-\sqrt{-1}$, we find that $\omega(n( \pm 2) \cdot w, 1) \varphi^{\prime \prime}(u)$ is equal to

$$
\begin{aligned}
2^{-3 / 2} \gamma_{V^{\prime \prime}}^{-1}[ & \varphi^{\prime \prime}\left(\mathbb{Z}_{2}, 2^{-2} \mathbb{Z}_{2}\right)-\varphi^{\prime \prime}\left(2^{-1} \mathbb{Z}_{2}^{\times}, 2^{-2} \mathbb{Z}_{2}\right) \\
& \left. \pm \delta \sqrt{-1} \varphi^{\prime \prime}\left(2^{-1} \mathbb{Z}_{2}^{\times}, 2^{-3} \mathbb{Z}_{2}^{\times}\right) \mp \delta \sqrt{-1} \varphi^{\prime \prime}\left(\mathbb{Z}_{2}, 2^{-3} \mathbb{Z}_{2}^{\times}\right)\right](u) .
\end{aligned}
$$

Note that

$$
\begin{aligned}
& \omega(w, 1) \varphi^{\prime \prime}\left(\mathbb{Z}_{2}, 2^{-2} \mathbb{Z}_{2}\right)=2^{-1 / 2} \gamma_{V^{\prime \prime}}^{-1} \\
& \times\left[\varphi^{\prime \prime}\left(\mathbb{Z}_{2}, 2^{-1} \mathbb{Z}_{2}\right)+\varphi^{\prime \prime}\left(2^{-1} \mathbb{Z}_{2}^{\times}, 2^{-1} \mathbb{Z}_{2}\right)+\varphi^{\prime \prime}\left(\mathbb{Z}_{2}, 2^{-2} \mathbb{Z}_{2}^{\times}\right)+\varphi^{\prime \prime}\left(2^{-1} \mathbb{Z}_{2}^{\times}, 2^{-2} \mathbb{Z}_{2}^{\times}\right)\right](u), \\
& \omega(w, 1) \varphi^{\prime \prime}\left(2^{-1} \mathbb{Z}_{2}^{\times}, 2^{-2} \mathbb{Z}_{2}\right)=2^{-1 / 2} \gamma_{V^{\prime \prime}}^{-1} \\
& \times\left[\varphi^{\prime \prime}\left(\mathbb{Z}_{2}, 2^{-1} \mathbb{Z}_{2}\right)-\varphi^{\prime \prime}\left(2^{-1} \mathbb{Z}_{2}^{\times}, 2^{-1} \mathbb{Z}_{2}\right)+\varphi^{\prime \prime}\left(\mathbb{Z}_{2}, 2^{-2} \mathbb{Z}_{2}^{\times}\right)-\varphi^{\prime \prime}\left(2^{-1} \mathbb{Z}_{2}^{\times}, 2^{-2} \mathbb{Z}_{2}^{\times}\right)\right](u), \\
& \omega(w, 1) \varphi^{\prime \prime}\left(2^{-1} \mathbb{Z}_{2}^{\times}, 2^{-3} \mathbb{Z}_{2}^{\times}\right)=2^{-1 / 2} \gamma_{V^{\prime \prime}}^{-1} \\
& \times\left[\varphi^{\prime \prime}\left(\mathbb{Z}_{2}, 2^{-1} \mathbb{Z}_{2}\right)-\varphi^{\prime \prime}\left(2^{-1} \mathbb{Z}_{2}^{\times}, 2^{-1} \mathbb{Z}_{2}\right)-\varphi^{\prime \prime}\left(\mathbb{Z}_{2}, 2^{-2} \mathbb{Z}_{2}^{\times}\right)+\varphi^{\prime \prime}\left(2^{-1} \mathbb{Z}_{2}^{\times}, 2^{-2} \mathbb{Z}_{2}^{\times}\right)\right](u), \\
& \omega(w, 1) \varphi^{\prime \prime}\left(\mathbb{Z}_{2}, 2^{-3} \mathbb{Z}_{2}^{\times}\right)=2^{-1 / 2} \gamma_{V^{\prime \prime}}^{-1} \\
& \times\left[\varphi^{\prime \prime}\left(\mathbb{Z}_{2}, 2^{-1} \mathbb{Z}_{2}\right)+\varphi^{\prime \prime}\left(2^{-1} \mathbb{Z}_{2}^{\times}, 2^{-1} \mathbb{Z}_{2}\right)-\varphi^{\prime \prime}\left(\mathbb{Z}_{2}, 2^{-2} \mathbb{Z}_{2}^{\times}\right)-\varphi^{\prime \prime}\left(2^{-1} \mathbb{Z}_{2}^{\times}, 2^{-2} \mathbb{Z}_{2}^{\times}\right)\right](u) .
\end{aligned}
$$

So we find that $\omega(w \cdot n( \pm 2) \cdot w, 1) \varphi^{\prime \prime}(u)$ is equal to

$$
2^{-1} \gamma_{V^{\prime \prime}}^{-2}\left[\varphi^{\prime \prime}\left(2^{-1} \mathbb{Z}_{2}^{\times}, 2^{-2} \mathbb{Z}_{2}\right) \pm \delta \sqrt{-1}\left(\varphi^{\prime \prime}\left(2^{-1} \mathbb{Z}_{2}^{\times}, 2^{-2} \mathbb{Z}_{2}^{\times}\right)-\varphi^{\prime \prime}\left(2^{-1} \mathbb{Z}_{2}^{\times}, 2^{-1} \mathbb{Z}_{2}\right)\right)\right](u) .
$$


Since $\gamma_{V^{\prime \prime}}^{2}=\chi_{V^{\prime \prime}}(-1)$, we find that $\omega(t(x) k, 1) \varphi^{\prime \prime}(u)$ is equal to

$$
\begin{aligned}
& 2^{-1} \chi_{V^{\prime \prime}}(x)|x|_{2} \\
& \times\left[\varphi^{\prime \prime}\left(2^{-1} \mathbb{Z}_{2}^{\times}, 2^{-2} \mathbb{Z}_{2}\right) \pm \delta \sqrt{-1}\left(\varphi^{\prime \prime}\left(2^{-1} \mathbb{Z}_{2}^{\times}, 2^{-2} \mathbb{Z}_{2}^{\times}\right)-\varphi^{\prime \prime}\left(2^{-1} \mathbb{Z}_{2}^{\times}, 2^{-1} \mathbb{Z}_{2}\right)\right)\right](u x) .
\end{aligned}
$$

This completes the proof.

Lemma 10.6. We put $l=\operatorname{ord}_{2}(\xi), m_{i}=\operatorname{ord}_{2}\left(b_{i}\right)$ for $i=1, \ldots, 4$,

$$
\begin{aligned}
& m_{0}=\min \left(\operatorname{ord}_{2}\left(b_{1}\right), \operatorname{ord}_{2}\left(b_{2}\right)+1, \operatorname{ord}_{2}\left(b_{3}\right)+d, \operatorname{ord}_{2}\left(b_{4}\right)\right), \\
& m_{0}^{\prime}=\min \left(\operatorname{ord}_{2}\left(b_{1}\right), \operatorname{ord}_{2}\left(b_{2}\right), \operatorname{ord}_{2}\left(b_{3}\right)+1, \operatorname{ord}_{2}\left(b_{4}\right)\right),
\end{aligned}
$$

and

$$
\begin{aligned}
& \delta_{2}= \begin{cases}1 & \text { if } m_{1}, m_{4} \geq m_{2}+1=m_{3}+2, \\
0 & \text { otherwise }\end{cases} \\
& \delta_{3}= \begin{cases}1 & \text { if } m_{1}, m_{2}, m_{4} \geq m_{3}+2 \text { and } \operatorname{ord}_{2}\left(b_{1} b_{4}-b_{2}^{2}\right)>\operatorname{ord}_{2}\left(D b_{3}^{2}\right), \\
0 & \text { otherwise }\end{cases} \\
& \delta_{0}= \begin{cases}1 & \text { if } m_{1}, m_{3}+2, m_{4} \geq m_{2}+1, \\
0 & \text { otherwise }\end{cases}
\end{aligned}
$$

(1) For $k=\mathbf{1}_{2}$, we have

$$
\begin{aligned}
& \Omega_{B, 2}\left(\mathbf{1}_{2}\right)=|\xi|_{2}^{\kappa+1 / 2} \sum_{n=0}^{m_{0}^{\prime}}\left(2^{n}\right)^{2 \kappa+1} a_{f}\left(\left(\xi 2^{-2 n}\right)_{2}\right), \\
& \Lambda_{B, 2}\left(\mathbf{1}_{2}\right)=|\xi|_{2}^{-s / 2+1 / 2} \sum_{n=0}^{\infty}\left|\xi 2^{2 n}\right|_{2}^{s / 2+2 \kappa+1} a_{f}\left(\left(\xi 2^{2 n}\right)_{2}\right) \overline{a_{g}\left(\left(\xi 2^{2 n}\right)_{2}\right)} .
\end{aligned}
$$

(2) For $k=-w \cdot n(-c) \cdot w$ with $c \in \mathbb{Z}_{2}^{\times}$or $k=n(a) \cdot w$ with $a \in 2 \mathbb{Z}_{2}$, we have

$$
\begin{aligned}
& \Omega_{B, 2}(k)=2^{-d} a_{f}\left(D_{2}\right)^{-1}|\xi|_{2}^{\kappa+1 / 2} \underline{\chi}_{2}(-\xi) \sum_{n=0}^{m_{0}}\left(2^{n}\right)^{2 \kappa+1} \overline{a_{f}\left(\left(D \xi 2^{-2 n}\right)_{2}\right)}, \\
& \Lambda_{B, 2}(k)=2^{-d} a_{f}\left(D_{2}\right)^{-1}|\xi|_{2}^{-s / 2+1 / 2} \underline{\chi}_{2}(-\xi C) \\
& \times \sum_{n=0}^{\infty}\left|\xi 2^{2 n}\right|_{2}^{s / 2+2 \kappa+1} \overline{a_{f}\left(\left(D \xi 2^{2 n}\right)_{2}\right) a_{g}\left(\left(\xi 2^{2 n}\right)_{2}\right)} .
\end{aligned}
$$

(3) For $k=-w \cdot n\left(-2^{d-1}\right) \cdot w=k_{1}$, we have

$$
\begin{aligned}
& \Omega_{B, 2}(k)=\delta_{d}|\xi|_{2}^{\kappa+1 / 2}\left(2^{\operatorname{ord}_{2}\left(b_{3}\right)+2}\right)^{2 \kappa+1} a_{f}(2)^{-1}, \\
& \Lambda_{B, 2}(k)=0 .
\end{aligned}
$$

(4) Assume that $d=3$. Let $k_{ \pm}=-w \cdot n( \pm 2) \cdot w$. Then we have

$$
\begin{aligned}
\Omega_{B, 2}\left(k_{+}\right)+\Omega_{B, 2}\left(k_{-}\right) & =\delta_{0}|\xi|_{2}^{\kappa+1 / 2}\left(2^{\operatorname{ord}_{2}\left(b_{2}\right)+1}\right)^{2 \kappa+1} a_{f}(2)^{-2}, \\
\Lambda_{B, 2}\left(k_{ \pm}\right) & =0 .
\end{aligned}
$$

Proof. (1) is easy. (2) follows from Lemma 10.5. So we show (3) and (4).

We show (3) only when $d=2$. Let $k=-w \cdot n(-2) \cdot w=k_{1}$. Then $\Omega_{B, 2}(k)$ is equal to

$$
\int_{\mathbb{Q}_{2}^{\times}}\left[\chi_{V}(x)|x|_{2}^{2} \varphi\left(2^{-1} \mathbb{Z}_{2}^{\times}, 2^{-2} \mathbb{Z}_{2}^{\times}\right)^{\wedge}\left(-\beta x ; 0, x^{-1}\right)\right] W_{\mathbf{f}, 2}\left(a(\xi) t(x) k_{1}\right)|x|_{2}^{-2} d^{\times} x .
$$

Let $x \in \mathbb{Q}_{2}^{\times}$and we put $n=\operatorname{ord}_{2}(x)$. Since $\beta={ }^{t}\left(-b_{4},-b_{2},-b_{3}, b_{1}\right)$, we have $\varphi\left(2^{-1} \mathbb{Z}_{2}^{\times}, 2^{-2} \mathbb{Z}_{2}^{\times}\right)^{\wedge}\left(-\beta x ; 0, x^{-1}\right) \neq$ 0 if and only if

$$
n \leq 0, \quad m_{4}+n \geq 0, \quad m_{2}+n=-1, \quad m_{3}+n=-2, \quad m_{1}+n \geq 0 .
$$


In this case, we find that $n=-\operatorname{ord}_{2}\left(b_{2}\right)-1=-\operatorname{ord}_{2}\left(b_{3}\right)-2$ and

$$
m_{1}, m_{4} \geq m_{2}+1=m_{3}+2 .
$$

Moreover, since $\operatorname{ord}_{2}\left(b_{2}\right)=\operatorname{ord}_{2}\left(2 b_{3}\right)$ and $D^{\prime}=D / 4 \equiv 1 \bmod 4$, we have

$$
\operatorname{ord}_{2}\left(b_{2}^{2}+D b_{3}^{2}\right)=\operatorname{ord}_{2}\left(b_{2}^{2}+D^{\prime}\left(2 b_{3}\right)^{2}\right)=2 \operatorname{ord}_{2}\left(b_{2}\right)+1<\operatorname{ord}_{2}\left(b_{1} b_{4}\right) \text {. }
$$

Hence, we have $l=\operatorname{ord}_{2}\left(b_{1} b_{4}-b_{2}^{2}-D b_{3}^{2}\right)=2 \operatorname{ord}_{2}\left(b_{2}\right)+1=-2 n-1$. So in this case, we have

$$
W_{\mathbf{f}, 2}\left(a(\xi) t(x) k_{1}\right)=\underline{\chi}_{2}\left(x^{-1}\right)\left|\xi x^{2}\right|_{2}^{\kappa+1 / 2} a_{f}(2)^{-1} .
$$

Therefore, we have

$$
\Omega_{B, 2}\left(k_{1}\right)=\delta_{2}|\xi|_{2}^{\kappa+1 / 2}\left(2^{-n}\right)^{2 \kappa+1} a_{f}(2)^{-1} .
$$

On the other hand, since $\Phi\left(t(x) k_{1}, s\right)=0$ for all $x \in \mathbb{Q}_{2}^{\times}$and $s \in \mathbb{C}$, we have $\Lambda_{B, 2}\left(k_{1}\right)=0$. The proof of (3) for $d=3$ is similar.

(4) We assume that $d=3$. Let $k_{ \pm}=-w \cdot n( \pm 2) \cdot w$. Then we find that $\Omega_{B, 2}\left(k_{ \pm}\right)$is equal to

$$
\begin{aligned}
\int_{\mathbb{Q}_{2}^{\times}} 2^{-1} \chi_{V}(x)|x|_{2}^{2}\left[\varphi\left(2^{-1} \mathbb{Z}_{2}^{\times}, 2^{-2} \mathbb{Z}_{2}\right) \pm \delta \sqrt{-1}\left(\varphi\left(2^{-1} \mathbb{Z}_{2}^{\times}, 2^{-2} \mathbb{Z}_{2}^{\times}\right)\right.\right. \\
\left.\left.-\varphi\left(2^{-1} \mathbb{Z}_{2}^{\times}, 2^{-1} \mathbb{Z}_{2}\right)\right)\right]^{\wedge}\left(-\beta x ; 0, x^{-1}\right) W_{\mathbf{f}, 2}\left(a(\xi) t(x) k_{ \pm}\right)|x|_{2}^{-2} d^{\times} x .
\end{aligned}
$$

Write $\xi=2^{l} \eta$ with $\eta \in \mathbb{Z}_{2}^{\times}$. We assume that $l+2 \in 2 \mathbb{Z}$ and put $n=(-l-2) / 2$. Then we have

$$
\begin{aligned}
\Omega_{B, 2}\left(k_{ \pm}\right) & =2^{-3 / 2} \underline{\chi}_{2}(\mp \eta) \varepsilon \zeta_{8}^{ \pm \eta}\left|\xi 2^{2 n}\right|_{2}^{\kappa+1 / 2} a_{f}(2)^{-2}\left[\varphi\left(2^{-1} \mathbb{Z}_{2}^{\times}, 2^{-2} \mathbb{Z}_{2}\right)\right. \\
& \left. \pm \delta \sqrt{-1}\left(\varphi\left(2^{-1} \mathbb{Z}_{2}^{\times}, 2^{-2} \mathbb{Z}_{2}^{\times}\right)-\varphi\left(2^{-1} \mathbb{Z}_{2}^{\times}, 2^{-1} \mathbb{Z}_{2}\right)\right)\right]^{\wedge}\left(-\beta 2^{n} ; 0,2^{-n}\right) .
\end{aligned}
$$

We put $\rho=\varepsilon\left(\underline{\chi}_{2}(-\eta) \zeta_{8}^{\eta}+\underline{\chi}_{2}(\eta) \zeta_{8}^{-\eta}\right)$ and $\rho^{\prime}=\varepsilon \sqrt{-1} \delta\left(\underline{\chi}_{2}(-\eta) \zeta_{8}^{\eta}-\underline{\chi}_{2}(\eta) \zeta_{8}^{-\eta}\right)$. Then by a simple calculation, we have

$$
\rho=\sqrt{2} \quad \text { and } \quad \rho^{\prime}= \begin{cases}\sqrt{2} & \text { if } \eta \equiv 1 \bmod 4 \mathbb{Z}_{2}, \\ -\sqrt{2} & \text { if } \eta \equiv 3 \bmod 4 \mathbb{Z}_{2} .\end{cases}
$$

Since $\varphi\left(2^{-1} \mathbb{Z}_{2}^{\times}, 2^{-2} \mathbb{Z}_{2}\right)=\varphi\left(2^{-1} \mathbb{Z}_{2}^{\times}, 2^{-2} \mathbb{Z}_{2}^{\times}\right)+\varphi\left(2^{-1} \mathbb{Z}_{2}^{\times}, 2^{-1} \mathbb{Z}_{2}\right)$, we find that $\Omega_{B, 2}\left(k_{+}\right)+\Omega_{B, 2}\left(k_{-}\right)$ is equal to

$$
\left|\xi 2^{2 n}\right|_{2}^{\kappa+1 / 2} a_{f}(2)^{-2} \times \begin{cases}\varphi\left(2^{-1} \mathbb{Z}_{2}^{\times}, 2^{-2} \mathbb{Z}_{2}^{\times}\right)^{\wedge}\left(-\beta 2^{n} ; 0,2^{-n}\right) & \text { if } \eta \equiv 1 \bmod 4 \mathbb{Z}_{2}, \\ \varphi\left(2^{-1} \mathbb{Z}_{2}^{\times}, 2^{-1} \mathbb{Z}_{2}\right)^{\wedge}\left(-\beta 2^{n} ; 0,2^{-n}\right) & \text { if } \eta \equiv 3 \bmod 4 \mathbb{Z}_{2} .\end{cases}
$$

However, if $\varphi\left(2^{-1} \mathbb{Z}_{2}^{\times}, 2^{-2} \mathbb{Z}_{2}^{\times}\right)^{\wedge}\left(-\beta 2^{n} ; 0,2^{-n}\right) \neq 0$, then we have

$$
n \leq 0, \quad m_{4}+n \geq 0, \quad m_{2}+n=-1, \quad m_{3}+n=-2, \quad m_{1}+n \geq 0 .
$$

In this case, we have

$$
l=\operatorname{ord}_{2}\left(b_{1} b_{4}-b_{2}^{2}-D b_{3}^{2}\right)=\operatorname{ord}_{2}\left(b_{2}^{2}\right)=-2 n-2 .
$$

So we have

$$
\begin{aligned}
\eta & =\xi 2^{-l}=4\left(2^{n} b_{1}\right)\left(2^{n} b_{4}\right)-\left(2^{-\operatorname{ord}_{2}\left(b_{2}\right)} b_{2}\right)^{2}-8 D^{\prime}\left(2^{n+1} b_{3}\right)^{2} \\
& \equiv-\left(2^{-\operatorname{ord}_{2}\left(b_{2}\right)} b_{2}\right)^{2}-2 \delta\left(2^{n+2} b_{3}\right)^{2} \bmod 4 \mathbb{Z}_{2} \\
& \equiv-1+2 \equiv 1 \bmod 4 \mathbb{Z}_{2} .
\end{aligned}
$$

Note that $u^{2} \equiv 1 \bmod 4 \mathbb{Z}_{2}$ for all $u \in \mathbb{Z}_{2}^{\times}$and $\pm 2 \equiv 2 \bmod 4 \mathbb{Z}_{2}$. Similarly, if $\varphi\left(2^{-1} \mathbb{Z}_{2}^{\times}, 2^{-1} \mathbb{Z}_{2}\right)^{\wedge}\left(-\beta 2^{n} ; 0,2^{-n}\right) \neq$ 0 , then we have $l=-2 n-2$ and $\eta \equiv-1 \bmod 4 \mathbb{Z}_{2}$. Therefore we have

$$
\begin{aligned}
\Omega_{B, 2}\left(k_{+}\right)+\Omega_{B, 2}\left(k_{-}\right) & =\left|\xi 2^{2 n}\right|_{2}^{\kappa+1 / 2} a_{f}(2)^{-2} \varphi\left(2^{-1} \mathbb{Z}_{2}^{\times}, 2^{-2} \mathbb{Z}_{2}\right)^{\wedge}\left(-\beta 2^{n} ; 0,2^{-n}\right) \\
& =\delta_{0}|\xi|_{2}^{\kappa+1 / 2}\left(2^{\operatorname{ord}_{2}\left(b_{2}\right)+1}\right)^{2 \kappa+1} a_{f}(2)^{-2} .
\end{aligned}
$$


On the other hand, since $\Phi\left(t(x) k_{ \pm}, s\right)=0$ for all $x \in \mathbb{Q}_{2}^{\times}$and $s \in \mathbb{C}$, we have $\Lambda_{B, 2}\left(k_{ \pm}\right)=0$. This completes the proof.

Now, we start to prove Lemma 6.7 (3) for the case when $p=2 \mid D$. First, we assume that $d=\operatorname{ord}_{2}(D)=2$. By Lemma 6.1 and Lemma 10.4, the function $\mathcal{W}_{B, 2}$ is right $\mathrm{GU}(2,2)\left(\mathbb{Z}_{2}\right)$ invariant. We put $q_{2}=\left(\mathrm{SL}_{2}\left(\mathbb{Z}_{2}\right): K_{0}\left(D ; \mathbb{Z}_{2}\right)\right)$. Then

$$
\begin{aligned}
\mathcal{W}_{B, 2}(1) & =q_{2}^{-1}|\xi|_{2}^{\kappa+1 / 2} a_{f}\left(D_{2}\right)^{-1}\left[a_{f}\left(D_{2}\right) \sum_{n=0}^{m_{0}^{\prime}}\left(2^{n}\right)^{2 \kappa+1} a_{f}\left(\left(\xi 2^{-2 n}\right)_{2}\right)\right. \\
& \left.+\sum_{n=0}^{m_{0}}\left(2^{n}\right)^{\kappa+1 / 2} \underline{\chi}_{2}(-\xi) \overline{a_{f}\left(\left(D \xi 2^{-2 n}\right)_{2}\right)}+a_{f}\left(D_{2}\right) \delta_{2}\left(2^{m_{3}+2}\right)^{2 \kappa+1} a_{f}(2)^{-1}\right] .
\end{aligned}
$$

For $0 \leq n \leq m_{0}^{\prime}$, we find that $\xi 2^{-2 n} \in \mathbb{Z}_{2}$. Note that

$$
m_{0}^{\prime}=\min \left(m_{1}, m_{2}, m_{3}+1, m_{4}\right) \leq m_{0}=\min \left(m_{1}, m_{2}+1, m_{3}+2, m_{4}\right)
$$

and $m_{0}^{\prime}=m_{0}$ if and only if $m_{0}^{\prime}=\min \left(m_{1}, m_{4}\right)$. In this case, we have $\delta_{2}=0$. So we consider the case when $m_{0} \neq m_{0}^{\prime}$. We divide the case into the three cases as follows.

If $m_{1}, m_{4}>m_{2}=m_{3}+1$, then we find that $\delta=1, m_{0}=m_{2}+1$ and

$$
l=\operatorname{ord}_{2}\left(b_{1} b_{4}-b_{2}^{2}-D b_{3}^{2}\right)=\operatorname{ord}_{2}\left(b_{2}^{2}+D^{\prime}\left(2 b_{3}\right)^{2}\right)=2 m_{2}+1 .
$$

So we have $\left(D \xi 2^{-2 m_{0}}\right)_{2}=2$. Moreover in this case we have

$$
a_{f}\left(D_{2}\right) \delta_{2}\left(2^{m_{3}+2}\right)^{2 \kappa+1} a_{f}(2)^{-1}=\left(2^{m_{0}}\right)^{2 \kappa+1} a_{f}(2) .
$$

If $m_{1}, m_{4}>m_{2}>m_{3}+1$, then we have $\delta_{2}=0, l=2\left(m_{3}+1\right)$ and $m_{0}=m_{3}+2$. So we have $\left(D \xi 2^{-2 m_{0}}\right)_{2}=1$. In this case, since

$$
-D \xi 2^{-2 m_{0}} \equiv\left(D b_{3} 2^{-m_{0}}\right)^{2} \equiv 1 \bmod 4 \mathbb{Z}_{2}
$$

and $\left(\mathbb{Z}_{2}^{\times}, 1+4 \mathbb{Z}_{2}\right)_{\mathbb{Q}_{2}}=1$, we have

$$
\underline{\chi}_{2}(-\xi)=(-D,-\xi)_{\mathbb{Q}_{2}}=\left(-D,-D \xi 2^{-2 m_{0}}\right)_{\mathbb{Q}_{2}}=\left(-D^{\prime},-D \xi 2^{-2 m_{0}}\right)_{\mathbb{Q}_{2}}=1 .
$$

If $m_{1}, m_{4}>m_{3}+1>m_{2}$, by the same calculation, we have $\delta=0, m_{0}=m_{2}+1$ and $\left(D \xi 2^{-2 m_{0}}\right)_{2}=1$. Moreover, since

$$
-D \xi 2^{-2 m_{0}} \equiv D^{\prime}\left(2 b_{2} 2^{-m_{0}}\right)^{2} \equiv 1 \bmod 4 \mathbb{Z}_{2},
$$

we have $\underline{\chi}_{2}(-\xi)=1$. This completes the proof of Lemma 6.7 (3) for the case when $p=2$ and $\operatorname{ord}_{2}(D)=2$. The proof of Lemma 6.7 (3) for the case when $p=2$ and $\operatorname{ord}_{2}(D)=3$ is similar.

Next, we start to prove Lemma 9.6 (3) for the case when $p=2 \mid D$. We put $q_{2}=\left(\mathrm{SL}_{2}\left(\mathbb{Z}_{2}\right)\right.$ : $\left.K_{0}\left(D ; \mathbb{Z}_{2}\right)\right)$. By Lemma 10.6, we have

$$
\begin{aligned}
I_{2}(\mathfrak{c}, s, \xi) & =q_{2}^{-1} a_{f}\left(D_{2}\right)^{-1}|\xi|_{2}^{-s / 2+1 / 2} \\
& \times \sum_{n=0}^{\infty}\left|\xi 2^{2 n}\right|_{2}^{s / 2+2 \kappa+1}\left[a_{f}\left(\left(D \xi 2^{2 n}\right)_{2}\right)+\underline{\chi}_{2}(-\xi C) \overline{a_{f}\left(\left(D \xi 2^{2 n}\right)_{2}\right)}\right] \overline{a_{g}\left(\left(\xi 2^{2 n}\right)_{2}\right)} .
\end{aligned}
$$

This is the desired formula for $I_{2}(\mathfrak{c}, s, \xi)$. By a calculation similar to that of $I_{2}(\mathfrak{c}, s, \xi)$, we find that $J_{2}\left(\mathfrak{c}, s, \xi, \xi^{\prime}\right)$ is equal to

$$
\begin{aligned}
& q_{2}^{-1}\left|\xi^{\prime}\right|_{2}^{\kappa+1 / 2}|\xi|_{2}^{\kappa+1}\left|a_{f}\left(D_{2}\right)\right|^{-1} \\
& \times \sum_{n=0}^{\infty}\left|2^{2 n}\right|_{2}^{\sigma / 2+2 \kappa+1}\left[\left|a_{f}\left(D_{2}\right) a_{f}\left(\left(\xi^{\prime} 2^{2 n}\right)_{2}\right)\right|+\left|\overline{a_{f}\left(\left(D \xi^{\prime} 2^{2 n}\right)_{2}\right)}\right|\right]\left|\overline{a_{g}\left(\left(\xi 2^{2 n}\right)_{2}\right)}\right| .
\end{aligned}
$$

Since $\left|a_{f}\left(D_{2}\right) a_{f}\left(\left(\xi^{\prime} 2^{2 n}\right)_{2}\right)\right| \leq\left|a_{f}\left(\left(D \xi^{\prime} 2^{2 n}\right)_{2}\right)\right|$ for all $\xi^{\prime} \in \mathbb{Q}^{\times}$, we get the desired estimation. This completes the proof of Lemma 9.6 (3) for the case when $p=2$. 
10.5. The case when $\operatorname{ord}_{p}(C) \neq 0$. Finally, we will prove Lemma 6.7 (4) and Lemma 9.6 (4).

Lemma 10.7. For $a \in \mathbb{Q}_{p}^{\times}$and $f \in L^{1}\left(\mathrm{SL}_{2}\left(\mathbb{Q}_{p}\right)\right)$, we have

$$
\int_{\mathrm{SL}_{2}\left(\mathbb{Q}_{p}\right)} f(x) d x=|a|_{p} \int_{B\left(\mathbb{Q}_{p}\right)} \int_{d(a) \mathrm{SL}_{2}\left(\mathbb{Z}_{p}\right) d(a)^{-1}} f(b k) d k d b .
$$

Proof. This lemma is proved by a change of variables immediately.

Now, we start to prove Lemma 6.7 (4). Let $\mathfrak{c}$ be an integral ideal of $K$. We assume that $\mathfrak{c}$ is prime to $2 D$ and $p \mid C=N(\mathfrak{c})$. Let $t \in \mathbb{A}_{K, \text { fin }}^{\times}$such that $\operatorname{ord}_{\mathfrak{p}}\left(t_{\mathfrak{p}}\right)=\operatorname{ord}_{\mathfrak{p}}(\mathfrak{c})$ for all prime ideals $\mathfrak{p}$ of $K$. Put $\gamma=r_{t}$. Write $t_{p}=x_{p} \otimes 1+y_{p} \otimes \sqrt{-D} \in \mathbb{Q}_{p} \otimes K$. Note that $\varphi_{p}$ is $\phi_{1}\left(\operatorname{GSU}(2,2)\left(\mathbb{Z}_{p}\right)\right) \phi_{2}\left(\left(\mathfrak{o} \otimes_{\mathbb{Z}} \mathbb{Z}_{p}\right)^{\times}\right)$-invariant by Lemma 8.2 . Therefore, by Lemma 6.1 , we only have to calculate $\mathcal{W}_{B, p}\left(\gamma_{p}\right)$. Recall that $\mathcal{W}_{B, p}\left(\gamma_{p}\right)$ is equal to

$$
\int_{N\left(\mathbb{Q}_{p}\right) \backslash \mathrm{SL}_{2}\left(\mathbb{Q}_{p}\right)} \hat{\omega}\left(\alpha \cdot d\left(N_{K / \mathbb{Q}}\left(t_{p}\right)\right), \phi_{2}\left(t_{p}\right)\right) \hat{\varphi}_{p}(-\beta ; 0,1) W_{\mathbf{f}, p}\left(a(\xi) \alpha \cdot d\left(N_{K / \mathbb{Q}}\left(t_{p}\right)\right)\right) d \alpha .
$$

Since the integrand is right $d\left(N_{K / \mathbb{Q}}\left(t_{p}\right)\right) \mathrm{SL}_{2}\left(\mathbb{Z}_{p}\right) d\left(N_{K / \mathbb{Q}}\left(t_{p}\right)\right)^{-1}$-invariant, by Lemma 10.7, we have

$$
\begin{aligned}
\mathcal{W}_{B, p}\left(\gamma_{p}\right)=\left|N_{K / \mathbb{Q}}\left(t_{p}\right)\right|_{p} \int_{\mathbb{Q}_{p}^{\times}} & \hat{\omega}\left(t(x) d\left(N_{K / \mathbb{Q}}\left(t_{p}\right)\right), \phi_{2}\left(t_{p}\right)\right) \hat{\varphi}_{p}(-\beta ; 0,1) \\
& \times W_{\mathbf{f}, p}\left(a(\xi) t(x) d\left(N_{K / \mathbb{Q}}\left(t_{p}\right)\right)\right)|x|_{p}^{-2} d^{\times} x .
\end{aligned}
$$

Note that $\left|N_{K / \mathbb{Q}}\left(t_{p}\right)\right|_{p}=|C|_{p}$. We have

$$
\begin{aligned}
& \hat{\omega}\left(t(x) d\left(N_{K / \mathbb{Q}}\left(t_{p}\right)\right), \phi_{2}(t)\right) \hat{\varphi}_{p}(-\beta ; 0,1) \\
& =\int_{\mathbb{Q}_{p}} \chi_{V}(x)|x|_{p}^{3}\left|N_{K / \mathbb{Q}}\left(t_{p}\right)\right|_{p}^{-3 / 2} \varphi_{p}\left(\phi_{2}\left(t_{p}\right)^{-1}\left(\begin{array}{c}
z x \\
-\beta x \\
0
\end{array}\right)\right) \psi(z) d z .
\end{aligned}
$$

Since

$$
\phi_{2}\left(t_{p}\right)^{-1}=N_{K / \mathbb{Q}}\left(t_{p}\right)^{-1}\left(\begin{array}{lll}
1 & & \\
& \phi^{\prime}\left(t_{p}\right) & \\
& & N_{K / \mathbb{Q}}\left(t_{p}\right)
\end{array}\right),
$$

we find that $\hat{\omega}\left(t(x) \cdot d\left(N_{K / \mathbb{Q}}\left(t_{p}\right)\right), \phi_{2}(t)\right) \hat{\varphi}_{p}(-\beta ; 0,1)$ is equal to

$$
\chi_{V}(x)|x|_{p}^{2}|C|_{p}^{-1 / 2} \hat{\varphi}_{p}\left(-N_{K / \mathbb{Q}}\left(t_{p}\right)^{-1} \phi^{\prime}\left(t_{p}\right) \beta x ; 0, N_{K / \mathbb{Q}}\left(t_{p}\right) x^{-1}\right) .
$$

On the other hand, we have

$$
\begin{aligned}
& W_{\mathbf{f}, p}\left(a(\xi) t(x) d\left(N_{K} / \mathbb{Q}\left(t_{p}\right)\right)\right) \\
& =\underline{\chi}_{p}\left(x^{-1} N_{K / \mathbb{Q}}\left(t_{p}\right)\right)\left|\xi x^{2} N_{K / \mathbb{Q}}\left(t_{p}\right)^{-1}\right|_{p}^{\kappa+1 / 2} a_{f}\left(\left(\xi x^{2} N_{K / \mathbb{Q}}\left(t_{p}\right)^{-1}\right)_{p}\right) \\
& =\underline{\chi}_{p}\left(x^{-1}\right)\left|\xi x^{2} N_{K / \mathbb{Q}}\left(t_{p}\right)^{-2} C\right|_{p}^{\kappa+1 / 2} a_{f}\left(\left(\xi x^{2} N_{K / \mathbb{Q}}\left(t_{p}\right)^{-2} C\right)_{p}\right) .
\end{aligned}
$$

Hence we have

$$
\begin{aligned}
\mathcal{W}_{B, p}\left(\gamma_{p}\right) & =|C|_{p}^{\kappa+1}|\xi|_{p}^{\kappa+1 / 2} \int_{\mathbb{Q}_{p}^{\times}} \hat{\varphi}_{p}\left(-\phi^{\prime}\left(t_{p}\right) \beta x ; 0, x^{-1}\right)\left|x^{2}\right|_{p}^{\kappa+1 / 2} a_{f}\left(\left(\xi x^{2} C\right)_{p}\right) d^{\times} x \\
& =|C|_{p}^{\kappa+1}|\xi|_{p}^{\kappa+1 / 2} \sum_{n=0}^{\infty} \hat{\varphi}_{p}\left(-\phi^{\prime}\left(t_{p}\right) \beta p^{-n} ; 0, p^{n}\right)\left(p^{n}\right)^{2 \kappa+1} a_{f}\left(\left(\xi C p^{-2 n}\right)_{p}\right)
\end{aligned}
$$

as desired. The last assertion of Lemma 6.7 (4) is easy.

Next, we start to prove Lemma 9.6 (4). Let $\mathfrak{c} \in J_{\mathfrak{o}}^{D}$. We assume that $C=N(\mathfrak{c})$ is a square free integer and $p \mid C$. Let $t \in \mathbb{A}_{K, \text { fin }}^{\times}$such that $\operatorname{ord}_{\mathfrak{p}}\left(t_{\mathfrak{p}}\right)=\operatorname{ord}_{\mathfrak{p}}(\mathfrak{c})$ for all prime ideals $\mathfrak{p}$ 
of $K$. Hence, we have $\left|N_{K / \mathbb{Q}}\left(t_{p}\right)\right|_{p}=|C|_{p}=p^{-1}$ and $C \cdot N_{K / \mathbb{Q}}\left(t_{p}\right)^{-1} \in \mathbb{Z}_{p}^{\times}$. Note that for $k^{\prime} \in K^{\prime}=d\left(N_{K / \mathbb{Q}}\left(t_{p}\right)\right) \mathrm{SL}_{2}\left(\mathbb{Z}_{p}\right) d\left(N_{K / \mathbb{Q}}\left(t_{p}\right)\right)^{-1}$, we have

$$
\begin{aligned}
& \Phi\left(t(x) k^{\prime}, s, L\left(\phi_{2}^{\prime \prime}\left(t_{p}\right)\right) \varphi_{p}^{\prime \prime}\right) \\
& =\chi_{V^{\prime \prime}}(x)|x|_{p}^{s+1}\left[\omega\left(k^{\prime} \cdot d\left(N_{K / \mathbb{Q}}\left(t_{p}\right)\right), \phi_{2}^{\prime \prime}\left(t_{p}\right)\right) \varphi_{p}^{\prime \prime}\right](0)\left|a\left(k^{\prime}\right)\right|_{p}^{s} \\
& =\chi_{V^{\prime \prime}}(x)|x|_{p}^{s+1}\left[L\left(\phi_{2}^{\prime \prime}\left(t_{p}\right)\right) \varphi_{p}^{\prime \prime}\right](0)\left|a\left(k^{\prime}\right)\right|_{p}^{s}=\chi_{V^{\prime \prime}}(x)|x|_{p}^{s+1}\left|N_{K / \mathbb{Q}}\left(t_{p}\right)\right|_{p}^{-1 / 2}\left|a\left(k^{\prime}\right)\right|_{p}^{s} .
\end{aligned}
$$

By Lemma $10.7, J_{p}\left(\mathfrak{c}, s, \xi, \xi^{\prime}\right)$ is equal to

$$
\begin{aligned}
& \int_{\mathbb{Q}_{p}^{\times}}\left(\left.\left.\left|N_{K / \mathbb{Q}}\left(t_{p}\right)\right|_{p} \int_{K^{\prime}}\left|\chi_{V^{\prime \prime}}(x)\right| x\right|_{p} ^{s+1}\left|N_{K / \mathbb{Q}}\left(t_{p}\right)\right|_{p}^{-1 / 2}\left|a\left(k^{\prime}\right)\right|\right|_{p} ^{s} \mid d k^{\prime}\right) \\
& \times\left|W_{\mathbf{f}, p}\left(a\left(\xi^{\prime} C\right) t(x) d\left(N_{K / \mathbb{Q}}\left(t_{p}\right)\right)\right) \overline{W_{\mathbf{g}, p}\left(a(\xi C) t(x) d\left(N_{K / \mathbb{Q}}\left(t_{p}\right)\right)\right)}\right||x|_{p}^{-2} d^{\times} x .
\end{aligned}
$$

Now $\left|a\left(k^{\prime}\right)\right|$ is right $\left(K^{\prime} \cap \mathrm{SL}_{2}\left(\mathbb{Z}_{p}\right)\right)$-invariant. Since $\operatorname{ord}_{p}\left(N_{K / \mathbb{Q}}\left(t_{p}\right)\right)=1$, a complete system of representatives of $K^{\prime} / K^{\prime} \cap \mathrm{SL}_{2}\left(\mathbb{Z}_{p}\right)$ is given by

$$
\left\{\left(\begin{array}{cc}
1 & b / p \\
0 & 1
\end{array}\right),\left(\begin{array}{cc}
0 & p^{-1} \\
-p & 0
\end{array}\right) \mid b \in \mathbb{Z}_{p} / p \mathbb{Z}_{p}\right\}
$$

Since

we have

$$
n(b / p) \in N\left(\mathbb{Q}_{p}\right) \text { and } \quad\left(\begin{array}{cc}
0 & p^{-1} \\
-p & 0
\end{array}\right)=\left(\begin{array}{cc}
p^{-1} & 0 \\
0 & p
\end{array}\right)\left(\begin{array}{cc}
0 & 1 \\
-1 & 0
\end{array}\right)
$$

Hence, we have

$$
\left|a\left(\begin{array}{cc}
1 & b / p \\
0 & 1
\end{array}\right)\right|=1 \text { and }\left|a\left(\begin{array}{cc}
0 & p^{-1} \\
-p & 0
\end{array}\right)\right|=\left|p^{-1}\right|_{p}=p .
$$

$$
\int_{K^{\prime}}\left|a\left(k^{\prime}\right)\right|^{s} d k=\frac{1}{p+1}\left(p+p^{s}\right)=\frac{p^{s}+p}{1+p}
$$

for $s \in \mathbb{C}$. Therefore we find that $J_{p}\left(\mathfrak{c}, s, \xi, \xi^{\prime}\right)$ is equal to

$$
\begin{aligned}
\left.p^{-1 / 2} \int_{\mathbb{Q}_{p}^{\times}}\left|\chi_{V^{\prime \prime}}(x)\right| x\right|_{p} ^{\sigma+1} \frac{p^{\sigma}+p}{1+p} \mid & \\
& \times\left.\left|\underline{\chi}_{p}\left(x^{-1} N_{K / \mathbb{Q}}\left(t_{p}\right)\right)\right| \xi^{\prime} C x^{2} N_{K / \mathbb{Q}}\left(t_{p}\right)^{-1}\right|_{p} ^{\kappa+1 / 2} a_{f}\left(\xi^{\prime} C x^{2} N_{K / \mathbb{Q}}\left(t_{p}\right)^{-1}\right) \mid \\
& \times\left|\overline{\left|\xi C x^{2} N_{K / \mathbb{Q}}\left(t_{p}\right)^{-1}\right|_{p}^{\kappa+1} a_{g}\left(\xi C x^{2} N_{K / \mathbb{Q}}\left(t_{p}\right)^{-1}\right)}\right||x|_{p}^{-2} d^{\times} x \\
= & p^{-1 / 2} \frac{p^{\sigma}+p}{1+p}\left|\xi^{\prime}\right|_{p}^{\kappa+1 / 2}|\xi|_{p}^{\kappa+1} \\
& \times \int_{\mathbb{Q}_{p}^{\times}}|x|_{p}^{\sigma+4 \kappa+2}\left|a_{f}\left(\xi^{\prime} C x^{2} N_{K / \mathbb{Q}}\left(t_{p}\right)^{-1}\right) \overline{a_{g}\left(\xi C x^{2} N_{K / \mathbb{Q}}\left(t_{p}\right)^{-1}\right)}\right| d^{\times} x \\
= & p^{-1 / 2} \frac{p^{\sigma}+p}{1+p}\left|\xi^{\prime}\right|_{p}^{\kappa+1 / 2}|\xi|_{p}^{\kappa+1} \sum_{n=0}^{\infty}\left(p^{-n}\right)^{\sigma+4 \kappa+2}\left|a_{f}\left(\xi^{\prime} p^{2 n}\right) \overline{a_{g}\left(\xi p^{2 n}\right)}\right| .
\end{aligned}
$$

This is the desired formula for $J_{p}\left(\mathfrak{c}, s, \xi, \xi^{\prime}\right)$. The formula for $I_{p}(\mathfrak{c}, s, \xi)$ is proved similarly. This completes the proof of Lemma 9.6 (4).

\section{REFERENCES}

[1] S. Böcherer, Über die Fourier-Jacobi-Entwicklung Siegelscher Eisensteinreihen. II, Math. Z. 189 (1985), no. 1, 81-110.

[2] W. Bosma, J. Cannon, and C. Playoust, The Magma algebra system. I. The user language, J. Symbolic Comput. 24 (1997), 235-265.

[3] C. J. Bushnell and G. Henniart, The Local Langlands Conjecture for GL(2), Springer-Verlag Berlin Heidelberg, 2006.

[4] T. Dokchitser, Computing special values of motivic L-functions, Experiment. Math. 13 (2004), no. 2, 137149. 
[5] M. Furusawa, On $L$-functions for $\mathrm{GSp}(4) \times \mathrm{GL}(2)$ and their special values. J. Reine Angew. Math. 438 (1993), 187-218.

[6] W. T. Gan, B. H. Gross and D. Prasad, Symplectic local root numbers, central critical $L$-values, and restriction problems in the representation theory of classical groups. Sur les conjectures de Gross et Prasad. I. Astérisque. No. 346 (2012), 1-109.

[7] W. T. Gan and A. Ichino, On endoscopy and the refined Gross-Prasad conjecture for $\left(\mathrm{SO}_{5}, \mathrm{SO}_{4}\right)$. J. Inst. Math. Jussieu 10 (2011), no. 2, 235-324.

[8] P. B. Garrett, Decomposition of Eisenstein series: Rankin triple products. Ann. of Math. (2) 125 (1987), no. 2, 209-235.

[9] B. H. Gross and D. Prasad, On the decomposition of a representation of $\mathrm{SO}_{n}$ when restricted to $\mathrm{SO}_{n-1}$. Can. J. Math. 44, (1992), 974-1002.

[10] M. Harris, L-functions and periods of polarized regular motives, J. Reine Angew. Math. 483 (1997), 75-161.

[11] M. Harris and S. S. Kudla, Arithmetic automorphic forms for the nonholomorphic discrete series of GSp(2), Duke Math. J. 66 (1992), no. 1, 59-121.

[12] E. Hecke, Lectures on the theory of algebraic numbers. Springer-Verlag, New York-Berlin, 1981.

[13] B. E. Heim, Pullbacks of Eisenstein series, Hecke-Jacobi theory and automorphic $L$-functions. Automorphic forms, automorphic representations, and arithmetic. Proc. Sympos. Pure Math. 66, Part 2, 201-238. Amer. Math. Soc., Providence, RI, 1999.

[14] A. Ichino, Pullbacks of Saito-Kurokawa lifts, Invent. math. 162 (2005), 551-647.

[15] A. Ichino, Trilinear forms and the central values of triple product $L$-functions. Duke Math. J. 145 (2008), no. 2, 281-307.

[16] A. Ichino and T. Ikeda, On Maass lifts and the central critical values of triple product $L$-functions, Amer. J. Math. 130 (2008), no. 1, 75-114.

[17] T. Ikeda, On the lifting of Hermitian modular forms, Compos. Math. 144 (2008), 1107-1154.

[18] H. Jacquet, Automorphic forms on GL(2). Part II. Lecture Notes in Mathematics, Vol. 278. Springer-Verlag, Berlin-New York, 1972.

[19] S. S. Kudla and S. Rallis, On the Weil-Siegel formula. J. Reine Angew. Math. 387 (1988), 1-68.

[20] T. Miyake, Modular Forms, Springer-Verlag, NY, 1989.

[21] K. Morimoto, On the theta correspondence for $\left(\mathrm{GSp}_{4}, \mathrm{GSO}_{4,2}\right)$ and Shalika periods. Represent. Theory 18 (2014), 28-87.

[22] A. Paul, On the Howe correspondence for symplectic-orthogonal dual pairs. J. Funct. Anal. 228 (2005), no. 2, 270-310.

[23] A. Saha, Pullbacks of Eisenstein series from GU(3,3) and critical $L$-values for GSp(4) $\times$ GL(2). Pacific J. Math. 246 (2010), no. 2, 435-486.

[24] G. Shimura, The special values of the zeta functions associated with cusp forms. Comm. Pure Appl. Math. 29 (1976), no. 6, 783-804.

[25] J. Tate, Number theoretic background. Automorphic forms, representations and L-functions. Proc. Sympos. Pure Math., Oregon State Univ., Corvallis, Ore., 1977, Part 2, pp. 3-26.

[26] J.-L. Waldspurger, Sur les valeurs de certaines fonctions $L$ automorphes en leur centre de symétrie. Compositio Math. 54 (1985), no. 2, 173-242.

[27] W. Zhang, Fourier transform and the global Gan-Gross-Prasad conjecture for unitary groups, Ann. of Math. (2) 180 (2014), no. 3, 971-1049.

[28] W. Zhang, Automorphic period and the central value of Rankin-Selberg L-function, J. Amer. Math. Soc. 27 (2014), no. 2, 541-612.

Department of mathematics, Kyoto University, Kitashirakawa-Oiwake-cho, Sakyo-ku, Kyoto, 6068502, JAPAN

E-mail address: atobe@math.kyoto-u.ac.jp 